Pacific Northwest

National Laboratory

Operated by Battelle for the

U.S. Department of Energy

\section{Borehole Data Package for Calendar Year 2001 RCRA Wells at Single-Shell Tank Waste Management Area U}

D. G. Horton

March 2002

Prepared for the U.S. Department of Energy under Contract DE-AC06-76RL01830 


\title{
DISCLAIMER
}

This report was prepared as an account of work sponsored by an agency of the United States Government. Reference herein to any specific commercial product, process, or service by trade name, trademark, manufacturer, or otherwise does not necessarily constitute or imply its endorsement, recommendation, or favoring by the United States Government or any agency thereof, or Battelle Memorial Institute.

\author{
PACIFIC NORTHWEST NATIONAL LABORATORY \\ operated by \\ BATTELLE \\ for the \\ UNITED STATES DEPARTMENT OF ENERGY \\ under Contract DE-AC06-76RL01830
}

This document was printed on recycled paper. 


\title{
Borehole Data Package for Calendar Year 2001 RCRA Wells at Single-Shell Tank Waste Management Area U
}

\author{
D. G. Horton
}

March 2002

Prepared for

the U.S. Department of Energy

under Contract DE-AC06-76RL01830

Pacific Northwest National Laboratory

Richland, Washington 99352 


\section{Summary}

This document is a compilation of the information on well drilling and construction, well development, pump installation, and sediment sampling at three new RCRA wells (299-W-18-40, 299-W19-44, and 299-W19-45) constructed at Waste Management Area U in September 2001. These wells were constructed to the specifications and requirements described in Washington Administrative Codes 173160 and 173-303.

Grab samples for geological description and archive were collected every $5 \mathrm{ft}$ throughout the wells. Borehole and drill cuttings were monitored regularly for organic vapors and radionuclide contaminants.

At well 299-W18-40, beta-gamma activity was found to be slightly above background at $120 \mathrm{ft}$ below ground surface. All other measurements were below background. Cesium-137 was found at the ground surface and at $3 \mathrm{ft}$ below ground surface (bgs). No other manmade contamination was found.

At well 299-W19-44, no radionuclide contamination was found, but several intervals of high carbon monoxide were detected. Cesium- 137 was detected at $3 \mathrm{ft}$ bgs at $0.4 \mathrm{pCi} / \mathrm{g}$.

At well 299-W19-45, no radionuclide contamination was found, but several intervals of high carbon monoxide were detected. Cesium- 137 was detected near the surface at $0.4 \mathrm{pCi} / \mathrm{g}$. No other manmade radionuclide was detected.

At well 299-W19-45, samples for geological description and archive were collected every $5 \mathrm{ft}$ throughout the well. No contamination was noted. Cesium-137 was detected near the surface at 0.4 to $1.4 \mathrm{pCi} / \mathrm{g}$. No other manmade radionuclide was detected. 


\section{Contents}

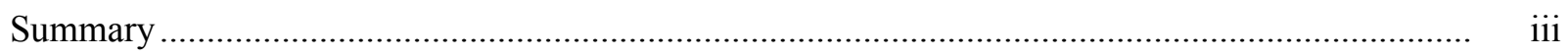

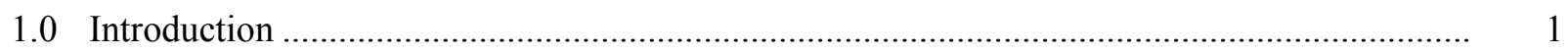

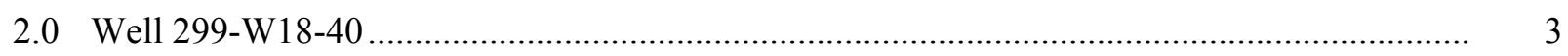

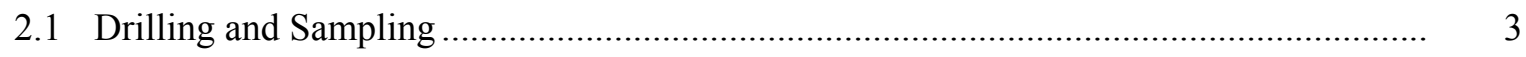

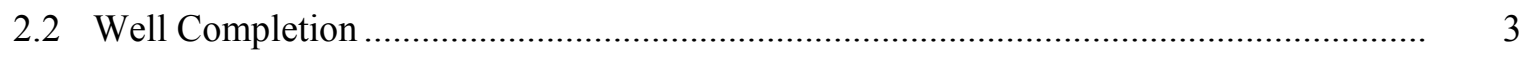

2.3 Well Development and Pump Installation.............................................................. 4

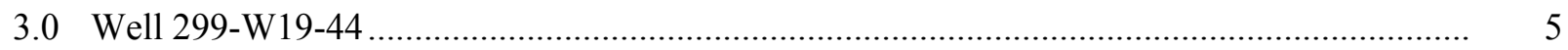

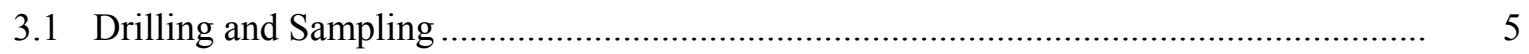

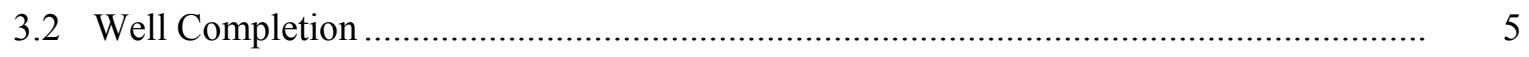

3.3 Well Development and Pump Installation................................................................ 6

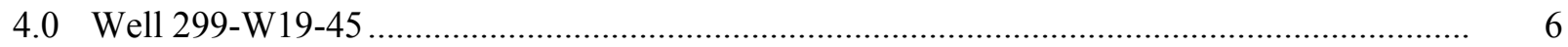

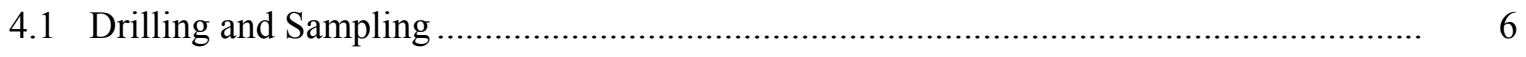

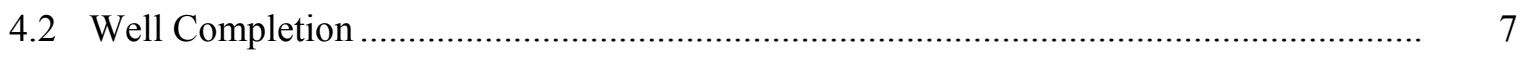

4.3 Well Development and Pump Installation............................................................... 7

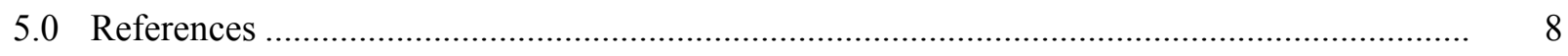

Appendix A - Well Construction and Completion Documentation ............................................ A.1

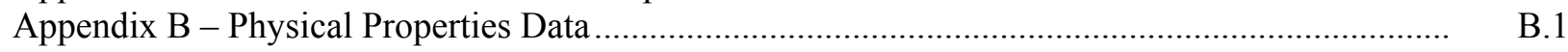

Appendix C - Borehole Geophysical Logs.................................................................... C.1 


\section{Figure}

1 Map of Waste Management Area U and Locations of Wells in the Groundwater

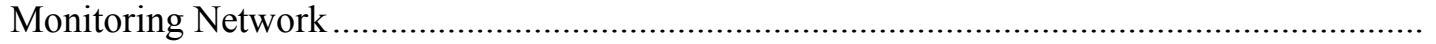

\section{Tables}

1 Well Names and Well Numbers for Wells Drilled at Waste Management Area U During Calendar Year 2001

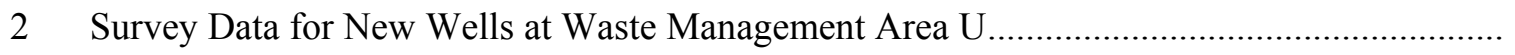




\subsection{Introduction}

Three new Resource Conservation and Recovery Act (RCRA) groundwater monitoring wells were installed at the single-shell tank farm Waste Management Area (WMA) U in August and September 2001 in partial fulfillment of Tri-Party Agreement (Ecology et al. 1998) milestone M-24-00M. The wells are 299-W18-40, 299-W19-44, and 299-W19-45. Table 1 correlates the well name with the well number. Well 299-W18-40 is located outside the southwest corner of 241-U tank farm and is a new upgradient well in the monitoring network. Wells 299-W19-44 and 299-W19-45 are new downgradient wells located east of the 241-U tank farm. The locations of all wells in the WMA U monitoring network are shown on Figure 1.

The groundwater quality assessment plan for WMA U was issued in 2001 and calls for the installation of five new wells (Smith et al. 2001). The three wells installed in 2001 are part of those described in the assessment plan. The new wells were constructed to the specifications and requirements described in Washington Administrative Codes 173-160 and 173-303, the assessment plan for WMA U (Smith et al. 2001), and the description of work for well drilling and construction. ${ }^{1}$

This document compiles information on the drilling and construction, well development, pump installation, and sediment sampling applicable to the installation of the three new wells. Appendix A contains the Well Summary Sheets (as-built diagrams), the Well Construction Summary Reports, and the geologist's logs; Appendix B contains results of physical properties testing; and Appendix C contains borehole geophysical logs. Additional documentation concerning well construction is on file with Bechtel Hanford, Inc., Richland, Washington.

English units are used in this report because that is the system of units used by drillers to measure and report depths and well construction details. The information below can be used for conversion to metric units:

- 1 foot $(\mathrm{ft})=0.3048$ meter

- 1 inch (in.) $=2.54$ centimeters

- 1 gallon $($ gal $)=3.785$ liters

Table 1. Well Names and Well Numbers for Wells Drilled at Waste Management Area U During Calendar Year 2001

\begin{tabular}{||l|l||}
\hline \multicolumn{1}{||}{ Well Name } & \multicolumn{1}{c||}{ Well Number } \\
\hline \hline 299-W18-40 & C3395 \\
\hline 299-W19-44 & C3393 \\
\hline 299-W19-45 & C3394 \\
\hline
\end{tabular}

\footnotetext{
${ }^{1}$ Letter from J. S. Fruchter (Pacific Northwest National Laboratory) to G. B. Mitchem (Bechtel Hanford Inc.) Description of Work for Drilling of CY 2001 RCRA Groundwater Monitoring Wells, dated April 16, 2001.
} 


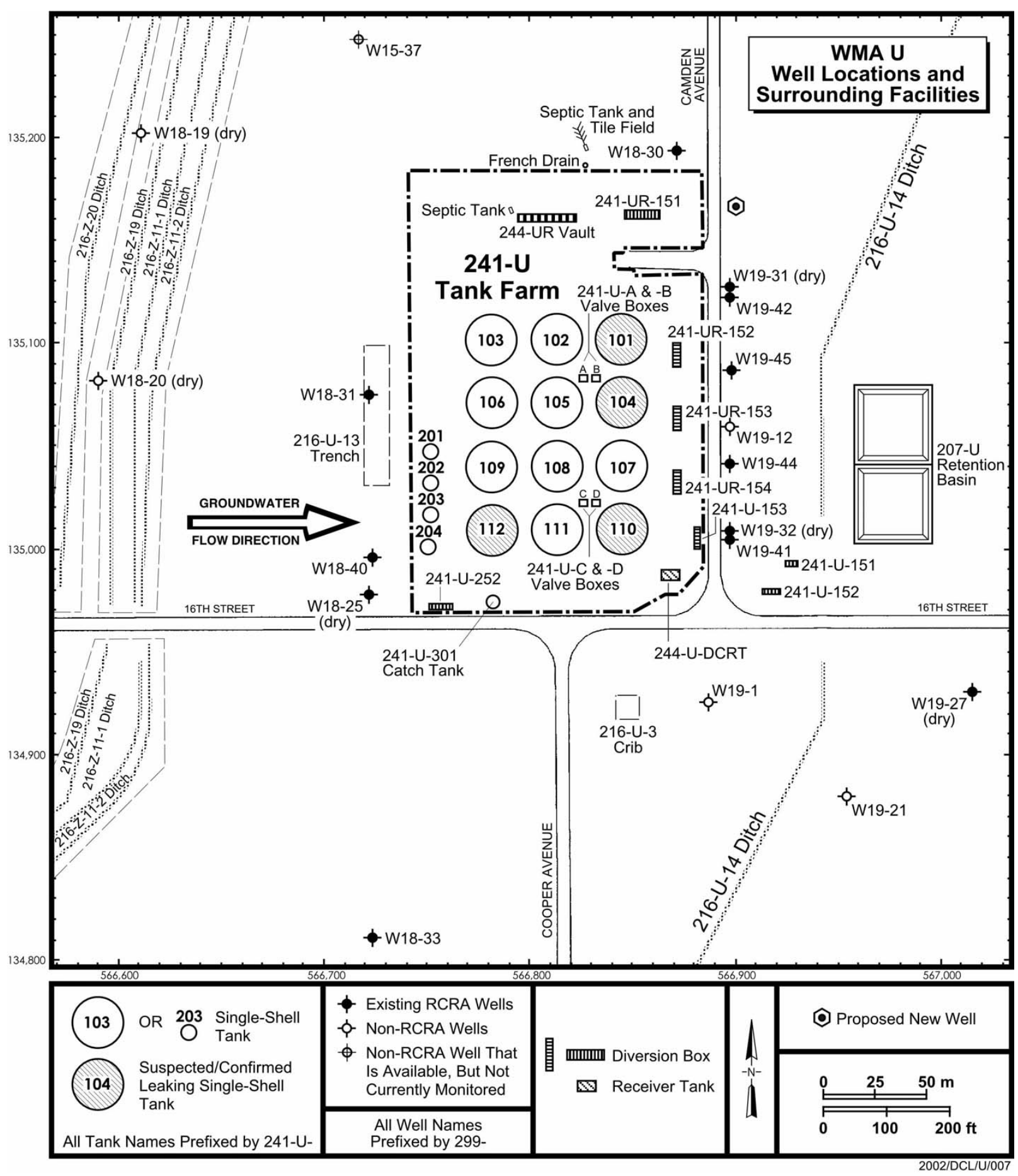

Figure 1. Map of Waste Management Area U and Locations of Wells in the Groundwater Monitoring Network 


\subsection{Well 299-W18-40}

\subsection{Drilling and Sampling}

Well 299-W18-40 was drilled in August and September 2001. The borehole was advanced with a cable tool rig and drive barrel from the surface to $147 \mathrm{ft}$ below ground surface (bgs). An air rotary drill rig was used from 147 to $178 \mathrm{ft}$ bgs and a cable tool rig with hard tool from $178 \mathrm{ft}$ to total depth of $260 \mathrm{ft}$ bgs. Temporary 10-3/4-in.-outside-diameter, carbon steel casing was used for the entire depth. An unknown amount of water was pumped into the borehole at about $178 \mathrm{ft}$ bgs to unstick the drill bit. Approximately 255 gal of water were added to the borehole during hard tool drilling.

The sediments encountered during drilling were eolian silty sand from the surface to about $10 \mathrm{ft}$ bgs and Hanford formation sandy gravel and silty sandy gravel from 10 to $\sim 69 \mathrm{ft}$ bgs and sand with minor silty sand and sandy silt from 69 to $116 \mathrm{ft}$ bgs. Sandy silt of the upper Plio-Pliestocene unit exists from about 116 to $133 \mathrm{ft}$ bgs and the lower Plio-Pleistocene silty sandy gravel with caliche from 133 to $137 \mathrm{ft}$ bgs. Sandy gravels and silty sandy gravels of the Ringold Formation member of Wooded Island, unit E exists from $137 \mathrm{ft}$ to the bottom of the borehole at $260 \mathrm{ft}$ bgs. The geologist's log is included in Appendix A.

Grab samples for geologic description and archive were collected every $5 \mathrm{ft}$ throughout the borehole. Also, two split spoon samples were taken from 220 to $222.5 \mathrm{ft}$ and from 250 to $252.5 \mathrm{ft}$ bgs for analysis of particle size distribution. Particle size distribution data are in Appendix B.

The borehole and drill cuttings were monitored regularly for organic vapors and radionuclide contaminants. Beta-gamma activity was found to be slightly above background at $120 \mathrm{ft}$ bgs. All other measurements were below background. The borehole was geophysically logged with spectral gamma and neutron moisture tools. Cesium- 137 was found at the ground surface and at $3 \mathrm{ft}$ bgs at $\leq 0.2 \mathrm{pCi} / \mathrm{g}$. No other manmade contamination was found.

\subsection{Well Completion}

The permanent casing and screen were installed in well 299-W18-40 in September 2001. A 4-in.inner-diameter, stainless steel, wire wrap, 20 slot screen was set from 253.28 to $218.27 \mathrm{ft}$ bgs. The permanent casing is 4-in.-inner-diameter, stainless steel from $218.27 \mathrm{ft}$ bgs to $1.9 \mathrm{ft}$ above ground surface. A 2-ft-long stainless steel sump is below the screen from a depth of 255.28 to $253.28 \mathrm{ft}$.

The filter pack is 10 to 20 mesh silica sand from 257.8 to $207.8 \mathrm{ft}$ bgs. The annular seal is $1 / 4$ in. bentonite pellets from 207.8 to $202.0 \mathrm{ft} \mathrm{bgs}$, granular bentonite from $202.0 \mathrm{ft}$ to $11.6 \mathrm{ft} \mathrm{bgs}$, and Portland cement grout from $11.6 \mathrm{ft}$ bgs to the surface. A $4 \mathrm{ft}$ by $4 \mathrm{ft}$ by $6 \mathrm{in}$. concrete pad was placed around the well at the surface. A protective casing with locking cap, four protective steel posts, and a brass marker 
stamped with the well number were set into the concrete. The protective casing extends $2.22 \mathrm{ft}$ above the concrete pad. The Well Summary Sheet (as-built) and Well Construction Summary Report are included in Appendix A.

The vertical and horizontal coordinates of the well were surveyed in December 2001. The horizontal position of the well was determined by Global Positioning System observations referenced to horizontal control stations established by Rogers Surveying, Inc., Richland, Washington and the U.S. Army Corps of Engineers. The coordinates are Washington Coordinate System, South Zone, NAD83(91) datum. Vertical datum is NAVD 1988 and is based on existing benchmarks established by the U.S. Army Corps of Engineers. Survey data are included in Table 2 and the survey data sheet is included in Appendix A.

Table 2. Survey Data for New Wells at Waste Management Area U

\begin{tabular}{||c|l|l|l|l||}
\hline Well Name & \multicolumn{1}{|c|}{ Easting $(\mathrm{m})$} & \multicolumn{1}{|c|}{ Northing $(\mathrm{m})$} & \multicolumn{1}{|c||}{ Elevation $(\mathrm{m})$} & \multicolumn{1}{|c||}{ Reference Point } \\
\hline \hline \multirow{3}{*}{ 299-W18-40 } & 566723.29 & 134996.41 & & Center of casing \\
\cline { 2 - 5 } & & & 203.413 & "X" on rim \\
\cline { 2 - 5 } & 566723.28 & 134996.72 & 202.735 & Brass cap \\
\hline \multirow{3}{*}{ 299-W19-44 } & 566896.95 & 135041.97 & & Center of casing \\
\cline { 2 - 6 } & & & 207.277 & "X" on rim \\
\cline { 2 - 6 } & 566896.95 & 135042.26 & 206.520 & Brass cap \\
\hline & 566897.65 & 135087.65 & & Center of casing \\
\cline { 2 - 6 } & & & 206.413 & "X" on rim \\
\cline { 2 - 6 } & 566897.64 & 135087.88 & 205.661 & Brass cap \\
\hline
\end{tabular}

\subsection{Well Development and Pump Installation}

Well 299-W18-40 was developed in September 2001. A temporary, submersible pump was used to remove approximately 1,067 gal of formation water at about $5 \mathrm{gal} / \mathrm{min}$. The pump intake was at $250 \mathrm{ft}$ bgs. Final drawdown was $12.1 \mathrm{ft}$ and turbidity was 4.46 NTU.

A dedicated submersible sampling pump (Redi-Flo2) was installed in well 299-W18-40 in October 2001. The sampling pump was originally installed with the intake at $34.95 \mathrm{ft}$ below static water level. The pump later was raised $20 \mathrm{ft}$. Static water level was $214.6 \mathrm{ft}$ bgs on September 27, 2001. 


\subsection{Well 299-W19-44}

\subsection{Drilling and Sampling}

Well 299-W19-44 was drilled in August and September 2001 with a cable tool drill rig from the surface to a total depth of $272.0 \mathrm{ft}$ bgs. The well was advance using drive barrel and split spoon sampler from the surface to a depth of $153 \mathrm{ft}$ and by hard tool from $153 \mathrm{ft}$ to total depth. Temporary 11-3/4-in.outside-diameter, carbon steel casing was used from the surface to $61 \mathrm{ft}$ bgs and $8-3 / 4-\mathrm{in}$. temporary casing from the surface to total depth of $272.0 \mathrm{ft}$.

The sediments encountered during drilling were Hanford formation sandy gravel and gravelly sand from the surface to $\sim 45 \mathrm{ft}$ bgs and sand and silty sand from 45 to $130 \mathrm{ft}$ bgs. Sandy silt of the upper PlioPleistocene exists from 130 to $144 \mathrm{ft}$ bgs and lower Plio-Pleistocene silty sandy gravel with caliche from 144 to $147 \mathrm{ft}$ bgs. Silty sandy gravel and sandy silty gravel of the Ringold Formation member of Wooded Island, unit E exists from $147 \mathrm{ft}$ bgs to total depth. The geologist's log is included in Appendix A.

Near continuous split spoon samples were collected from the surface to $145 \mathrm{ft}$ bgs for characterization of uncontaminated vadose zone sediments. Grab samples for geologic description and archive were collected every $5 \mathrm{ft}$ throughout the borehole. Also, two split spoon samples were taken from 232.0 to $234.5 \mathrm{ft}$ and from 267 to $269.5 \mathrm{ft}$ bgs for analysis of particle size distribution. Particle size distribution data are in Appendix B.

The borehole and drill cuttings were monitored regularly for organic vapors and radionuclide contaminants. No radionuclide contamination was found by field screening methods but several intervals had relatively high carbon monoxide levels in the borehole. The borehole was geophysically logged with spectral gamma-ray and neutron moisture tools on September 4 and 5, 2001. Cesium-137 was the only manmade radionuclide identified. Cesium- 137 was found at $3 \mathrm{ft}$ bgs at $0.4 \mathrm{pCi} / \mathrm{g}$.

\subsection{Well Completion}

The permanent casing and screen were installed in well 299-W19-44 in September 2001. A 4-in.inner-diameter, stainless steel, wire wrap, 20 slot screen was set from 264.9 to $229.9 \mathrm{ft}$ bgs. The permanent casing is 4-in.-inner-diameter, stainless steel from $229.9 \mathrm{ft}$ bgs to $2.0 \mathrm{ft}$ above ground surface. A 2-ft-long stainless steel sump is below the screen from 266.9 to $264.9 \mathrm{ft}$ bgs.

The filter pack is 10 to 20 mesh silica sand from 272.0 to $218.9 \mathrm{ft}$ bgs. The annular seal is $1 / 4-\mathrm{in}$. bentonite pellets from 218.9 to $213.03 \mathrm{ft}$ bgs, bentonite crumbles from 213.03 to $10.3 \mathrm{ft}$ bgs, and Portland cement grout from $10.3 \mathrm{ft}$ bgs to the surface. A $4 \mathrm{ft}$ by $4 \mathrm{ft}$ by 6 in. concrete pad was placed around the well at the surface. A 6-in. stainless steel protective casing with locking cap, four protective steel posts, 
and a brass marker stamped with the well number were set into the concrete. The protective casing extends $2.48 \mathrm{ft}$ above the concrete pad. The Well Summary Sheet (as-built) and Well Construction Summary Report are included in Appendix A.

The vertical and horizontal coordinates of the well were surveyed in December 2001. The horizontal position of the well was determined by Global Positioning System observations referenced to horizontal control stations established by Rogers Surveying, Inc., Richland, Washington and the U.S. Army Corps of Engineers. The coordinates are Washington Coordinate System, South Zone, NAD83(91) datum. Vertical datum is NAVD 1988 and is based on existing benchmarks established by the U.S. Army Corps of Engineers. Survey data are included in Table 2 and the survey data sheet is included in Appendix A.

\subsection{Well Development and Pump Installation}

Well 299-W19-44 was developed in September 2001. A temporary, $3 \mathrm{hp}$, submersible pump was used to remove approximately $936 \mathrm{gal}$ of formation water from $263 \mathrm{ft}$ bgs. Well development began at $5 \mathrm{gal} / \mathrm{min}$ but excessive drawdown $(27 \mathrm{ft})$ dictated a slower pumping rate of $3 \mathrm{gal} / \mathrm{min}$ for most of the development. The final drawdown was $18.6 \mathrm{ft}$ and the final turbidity was $3 \mathrm{NTU}$. The top half of the screened interval was not developed. The final turbidity was 1.81 NTU.

A dedicated, Redi Flo-2 submersible sampling pump was installed in well 299-W19-44 in September 2001. The sampling pump intake is at $255.35 \mathrm{ft}$ bgs (or about $28.4 \mathrm{ft}$ below the water table). Static water level was $226.96 \mathrm{ft}$ bgs on September 14, 2001.

\subsection{Well 299-W19-45}

\subsection{Drilling and Sampling}

Well 299-W19-45 was drilled with a cable tool drill rig from the surface to $30 \mathrm{ft}$ bgs and with air rotary rig from $30 \mathrm{ft}$ to a total depth of $266.1 \mathrm{ft} \mathrm{bgs} \mathrm{during} \mathrm{August} \mathrm{2001.} \mathrm{Temporary} \mathrm{10-3/4-in.-outside-}$ diameter, carbon steel casing was placed from the surface to total depth. About 75 gal of water were added at $233 \mathrm{ft}$ bgs to clear cuttings from the borehole, and an unknown amount of water was added at $\sim 256 \mathrm{ft}$ to keep the cyclone sampler from plugging.

Preliminary evaluation shows that the sediments encountered during drilling were Hanford formation silty sandy gravel and sandy gravel from the surface to about $53 \mathrm{ft}$ bgs and sand, gravelly sand and silty sand from 53 to $133 \mathrm{ft}$ bgs. Sandy silt of the upper Plio-Pleistocene exists from 133 to $137 \mathrm{ft}$ bgs and lower Plio-Pleistocene sand and silt and silty sandy gravel with caliche exists from 137 to $145 \mathrm{ft}$ bgs. The Ringold Formation member of Wooded Island, unit E was encountered from $145 \mathrm{ft}$ bgs to the bottom of the borehole. The geologist's log is in Appendix A. 
Sediment samples were collected at approximately 5-ft intervals for geologic description and archive throughout the entire borehole. Two split spoon samples were collected from 224 to $226 \mathrm{ft}$ and from 257 to $259 \mathrm{ft}$ bgs for analysis of grain size distribution. Data are in Appendix B.

The borehole and drill cuttings were monitored regularly for organic vapors and radionuclide contaminants. No contamination was noted by field screening methods. The borehole was geophysically logged with spectral gamma-ray and neutron moisture tools on August 15 and 16, 2001. Cesium-137 was identified near the surface at 0.4 to $1.4 \mathrm{pCi} / \mathrm{g}$. No other manmade radioisotopes were found. The geophysical logs are in Appendix C.

\subsection{Well Completion}

The permanent casing and screen were installed in well 299-W19-45 in August 2001. A 4-in.-insidediameter, stainless steel, continuous wire wrap (20 slot) screen was set from 259.03 to $224.12 \mathrm{ft}$ bgs. The permanent casing is 4-in.-inside-diameter stainless steel from $224.12 \mathrm{ft}$ bgs to $2.11 \mathrm{ft}$ above ground surface. A 2-ft-long sump from 261.13 to $259.03 \mathrm{ft}$ is attached to the bottom of the screen.

The filter pack is 10 to 20 mesh silica sand from 266.1 to $213.4 \mathrm{ft} \mathrm{bgs}$. The annular seal is bentonite pellets from 213.4 to $207.3 \mathrm{ft}$ bgs, bentonite crumbles from 207.3 to $9.5 \mathrm{ft} \mathrm{bgs}$, and Portland cement from $9.5 \mathrm{ft}$ bgs to the surface. A $4 \mathrm{ft}$ by $4 \mathrm{ft}$ by 6 in. concrete pad was placed around the well at the surface. A 6-in. stainless steel protective casing with locking cap, four protective steel posts, and a brass marker stamped with the well number were set into the concrete. The Well Summary Sheet (as-built) and Well Construction Summary Report are included in Appendix A.

The vertical and horizontal coordinates of the well were surveyed in December 2001. The horizontal position of the well was determined by Global Positioning System observations referenced to horizontal control stations established by Rogers Surveying, Inc., Richland, Washington and the U.S. Army Corps of Engineers. The coordinates are Washington Coordinate System, South Zone, NAD83(91) datum. Vertical datum is NAVD 1988 and is based on existing benchmarks established by the U.S. Army Corps of Engineers. Survey data are included in Table 2 and the survey data sheet is included in Appendix A.

\subsection{Well Development and Pump Installation}

Well 299-W19-45 was developed in August 2001. A temporary, $1 \mathrm{hp}$, submersible pump was used to remove approximately about 3,940 gal of formation water. First, about 3,540 gal of water were removed at $30 \mathrm{gal} / \mathrm{min}$ with the pump intake at $257.5 \mathrm{ft}$ bgs resulting in $17.3 \mathrm{ft}$ of drawdown. Then about 400 gal of water were removed at $10 \mathrm{gal} / \mathrm{min}$ with the pump intake at $236.5 \mathrm{ft}$ bgs; drawdown was about $4.4 \mathrm{ft}$. The final turbidity was 1.20 NTU.

A dedicated, Redi Flo-2 submersible sampling pump was installed in well 299-W19-45 in September 2001. The sampling pump intake is at $235.35 \mathrm{ft}$ below the top of casing ( $8.5 \mathrm{ft}$ below the water table). Static water level in the well was $226.85 \mathrm{ft}$ below top of casing on September 4, 2001. 


\subsection{References}

Ecology - Washington State Department of Ecology, U.S. Environmental Protection Agency, and U.S. Department of Energy. 1998. Hanford Federal Facility Agreement and Consent Order. Document No. 89-10, Rev. 5 (The Tri-Party Agreement), Ecology, Olympia, Washington.

NAVD88. 1988. North American Vertical Datum of 1988.

RCRA - Resource Conservation and Recovery Act. 1976. Public Law 94-580, as amended, 90 Stat. 2795, 42 USC 6901 et seq.

Smith, R. M., F. N. Hodges, and B. A. Williams. 2001. Groundwater Quality Assessment Plan for Single-Shell Tank Farm Waste Management Area U. PNNL-13612, Pacific Northwest National Laboratory, Richland, Washington.

WAC 173-160, Washington Administrative Code. Minimum Standards for Construction and Maintenance of Wells. Olympia, Washington.

WAC 173-303, Washington Administrative Code. Dangerous Waste Regulations. Olympia, Washington. 


\section{Appendix A}

\section{Well Construction and Completion Documentation}




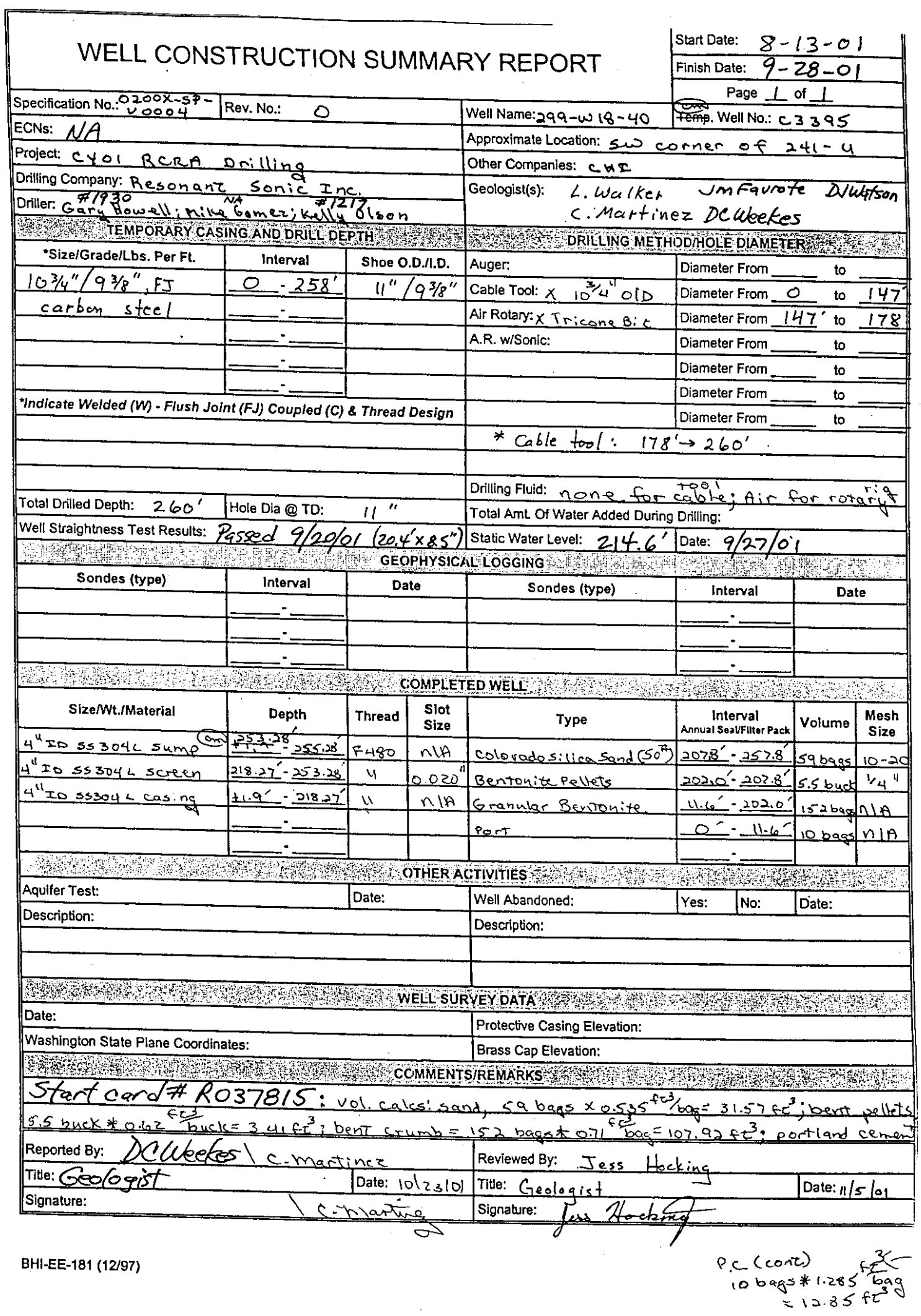




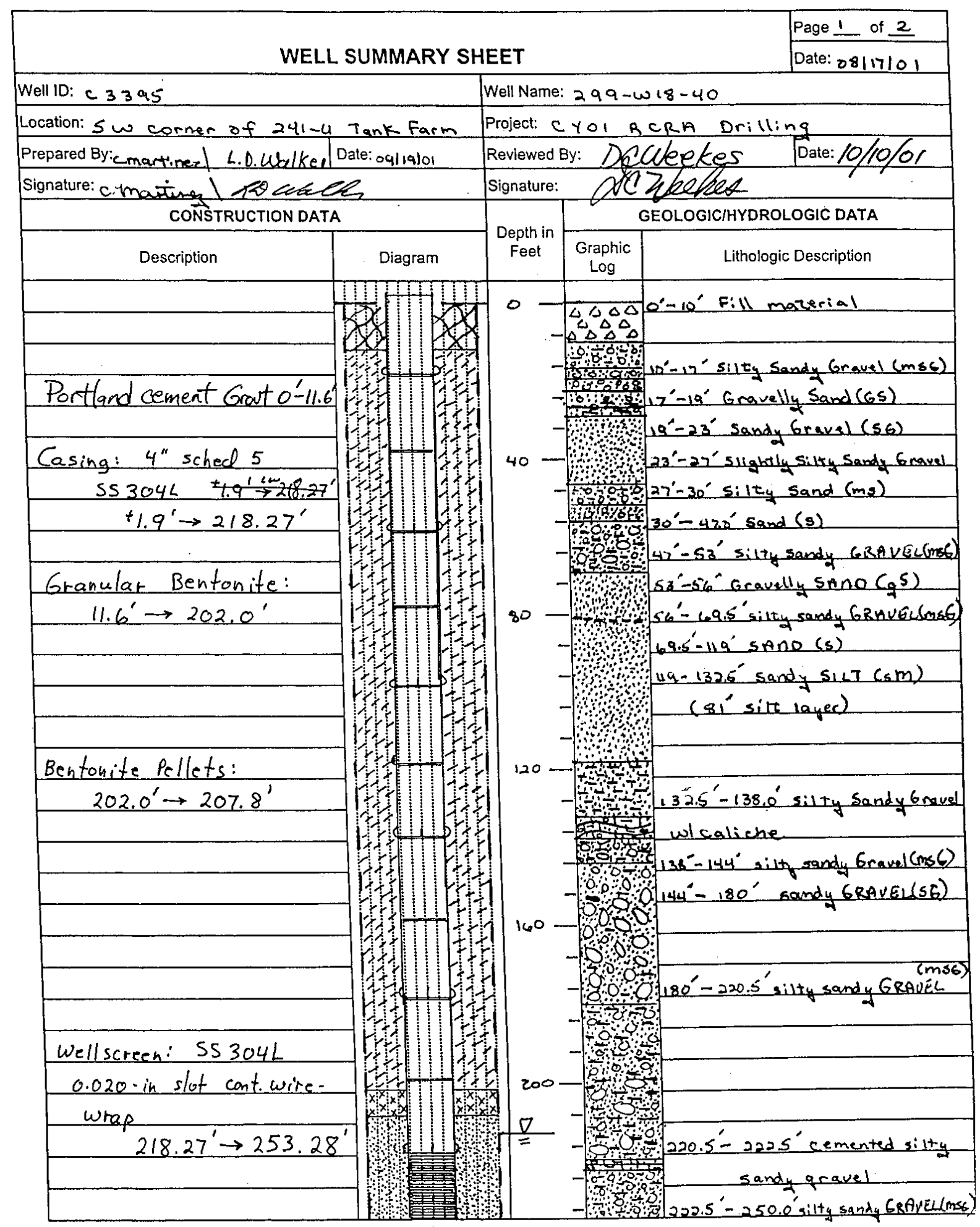

BHI-EE-189 (12/97) 


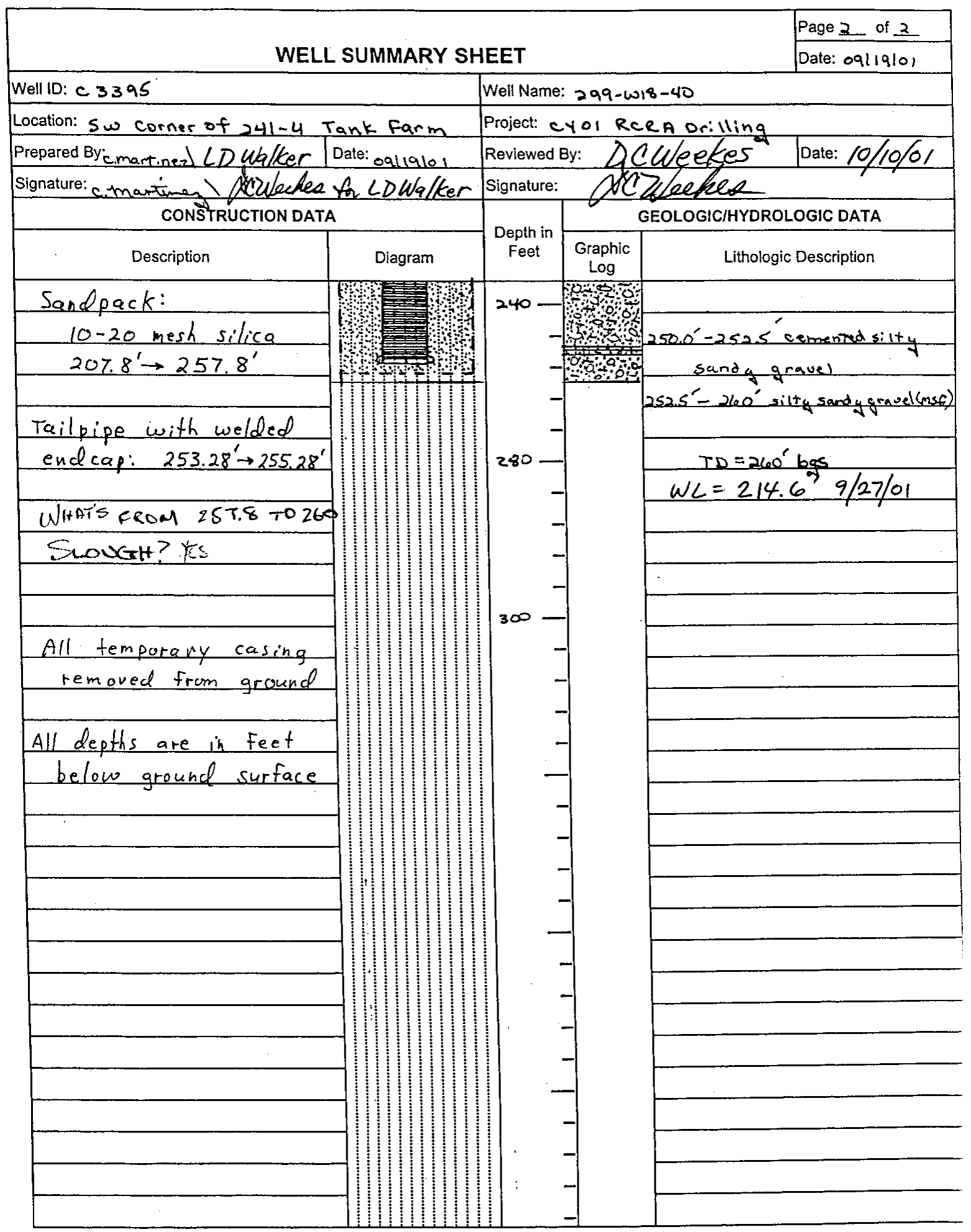

BHI-EE-189 (12/97) 


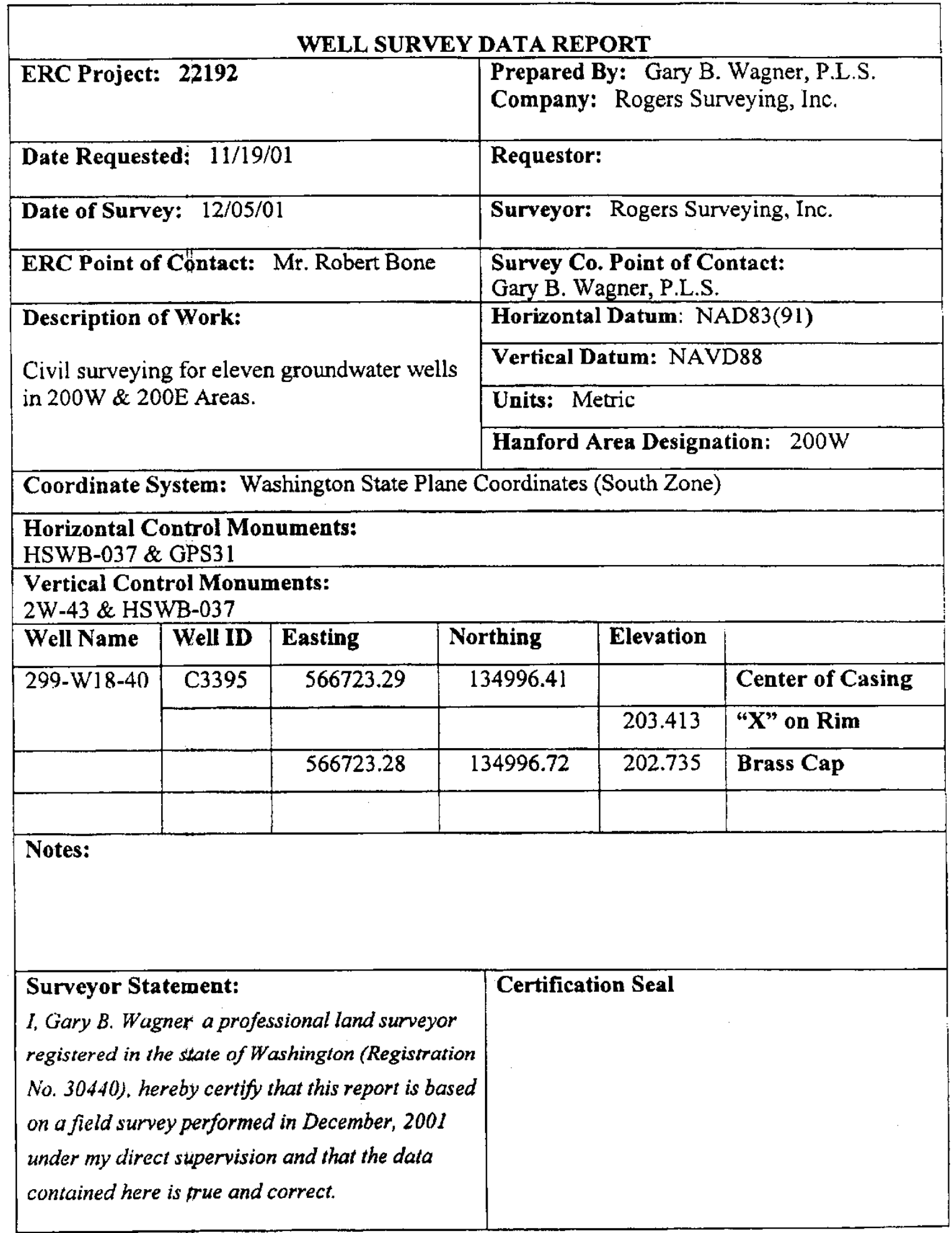

BHI-EE-202 (09/98) 


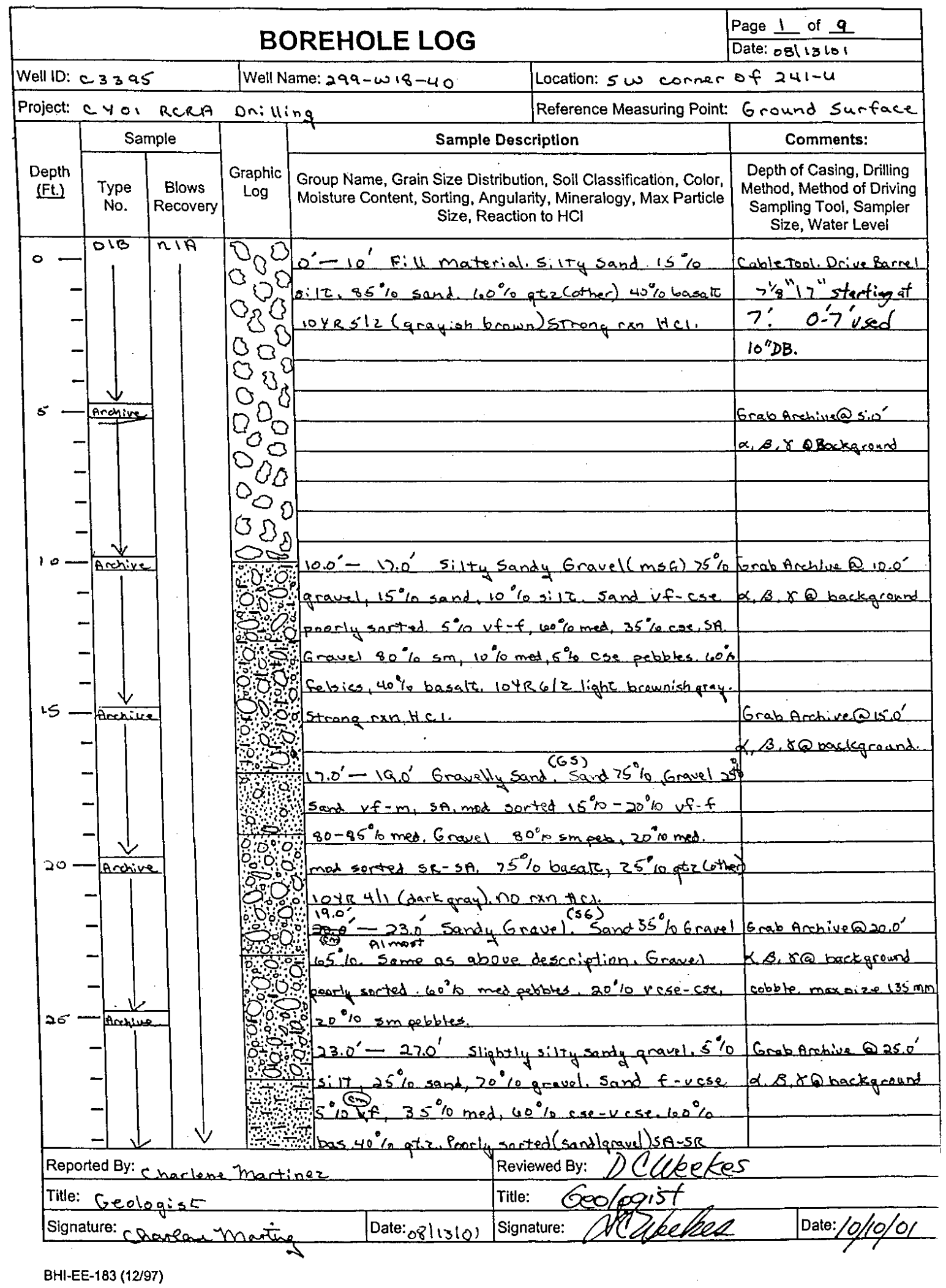




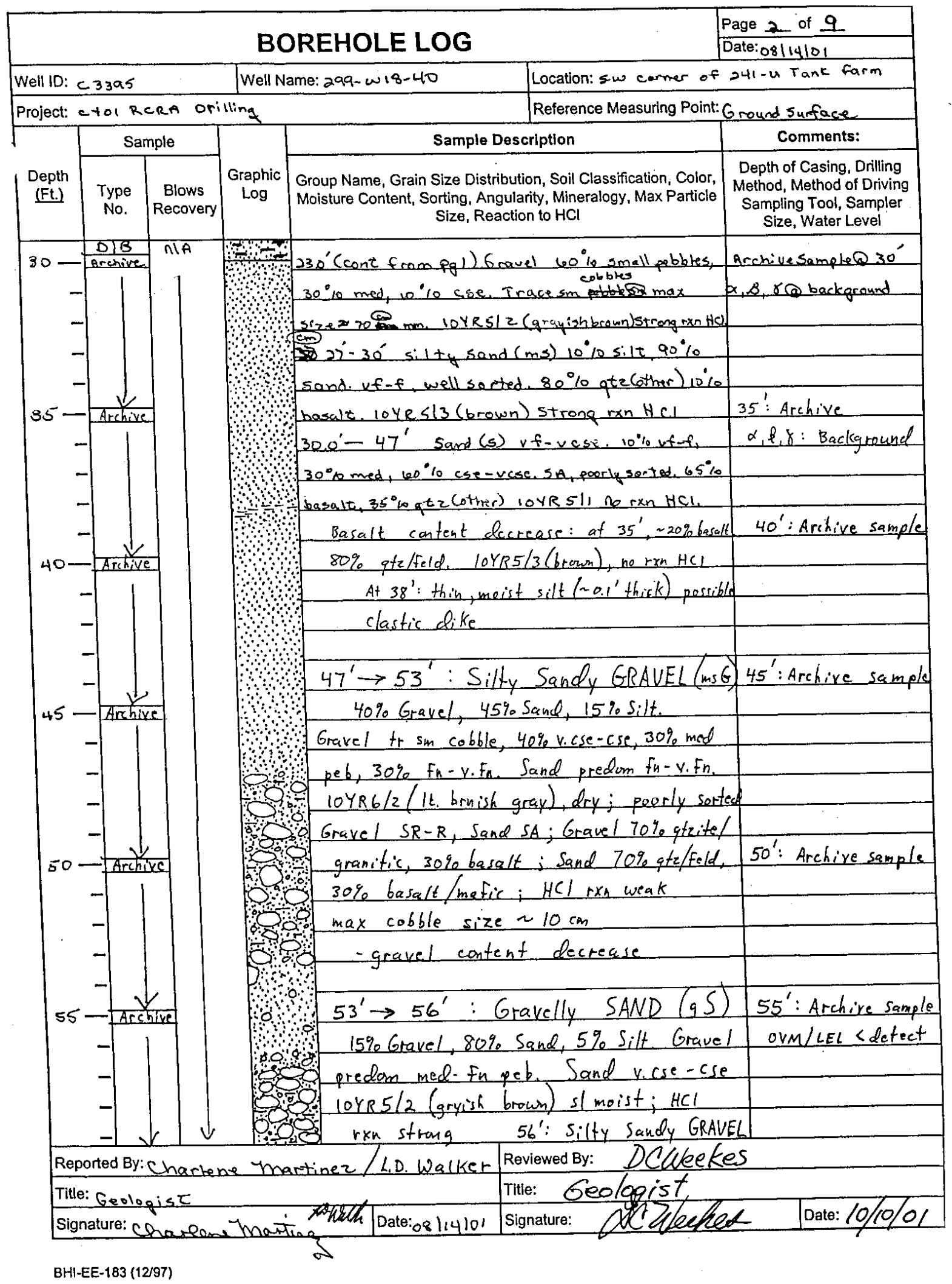




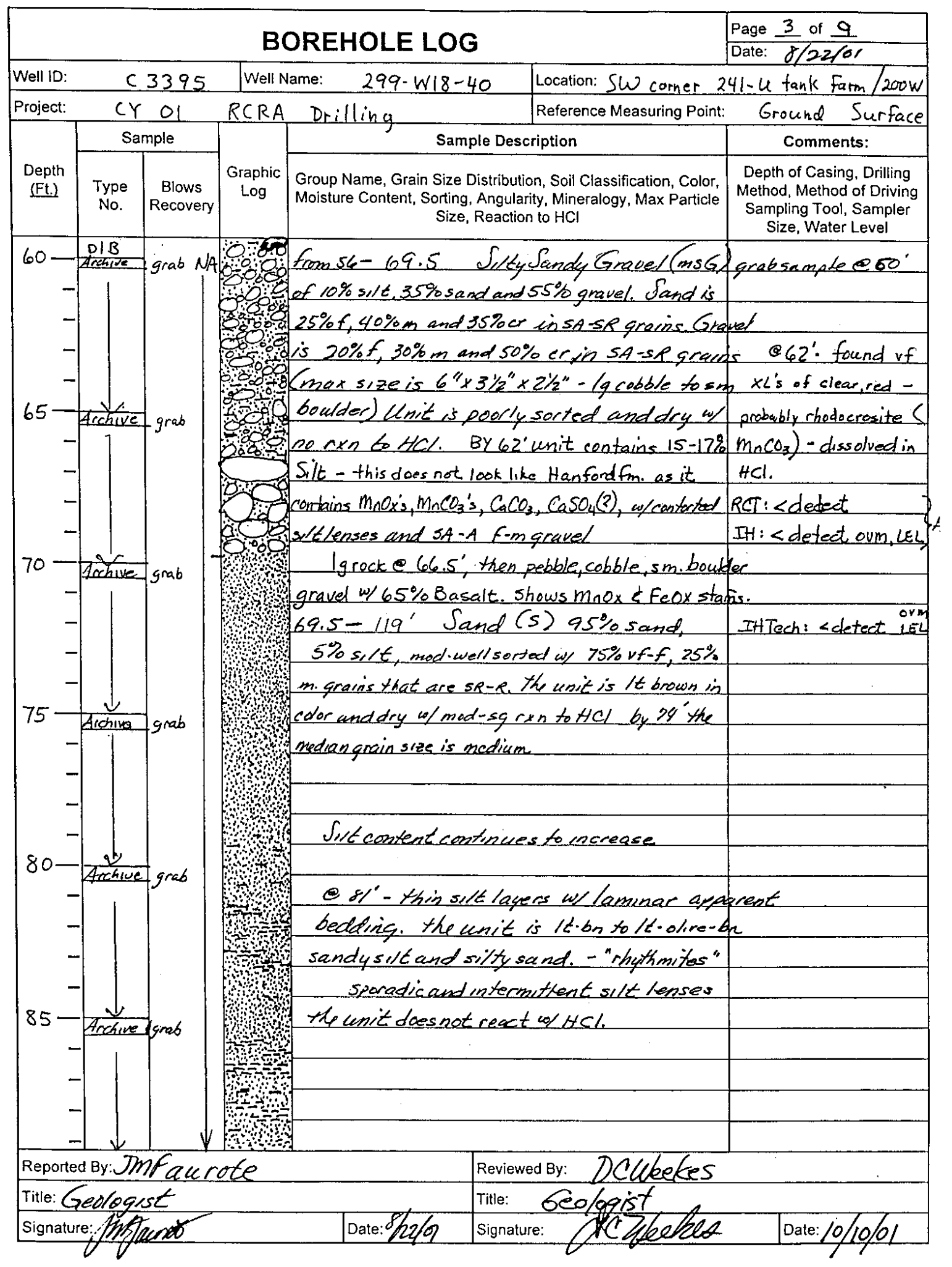

BHI-EE-183 (12/97) 


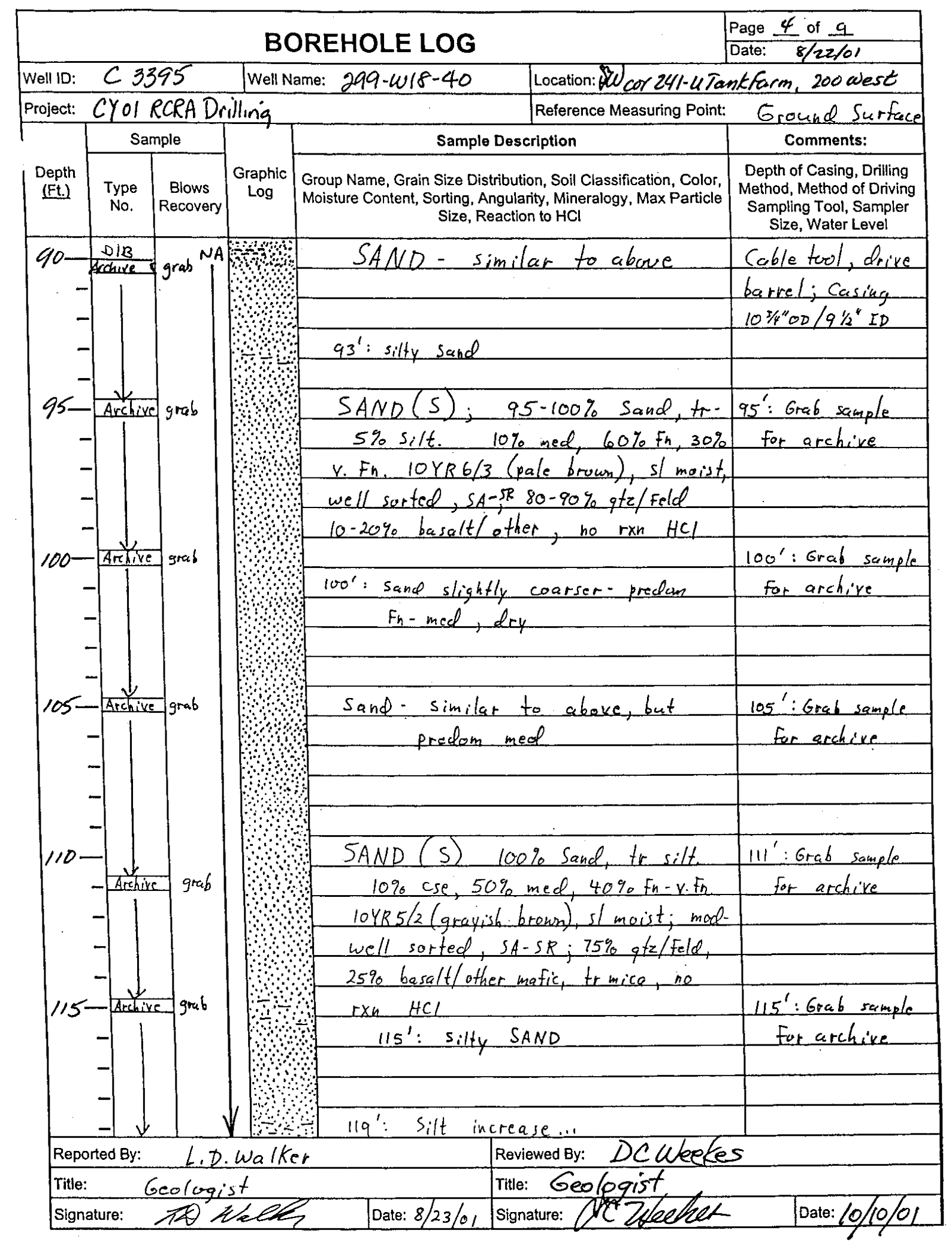

BHI-EE-183 (12/97) 


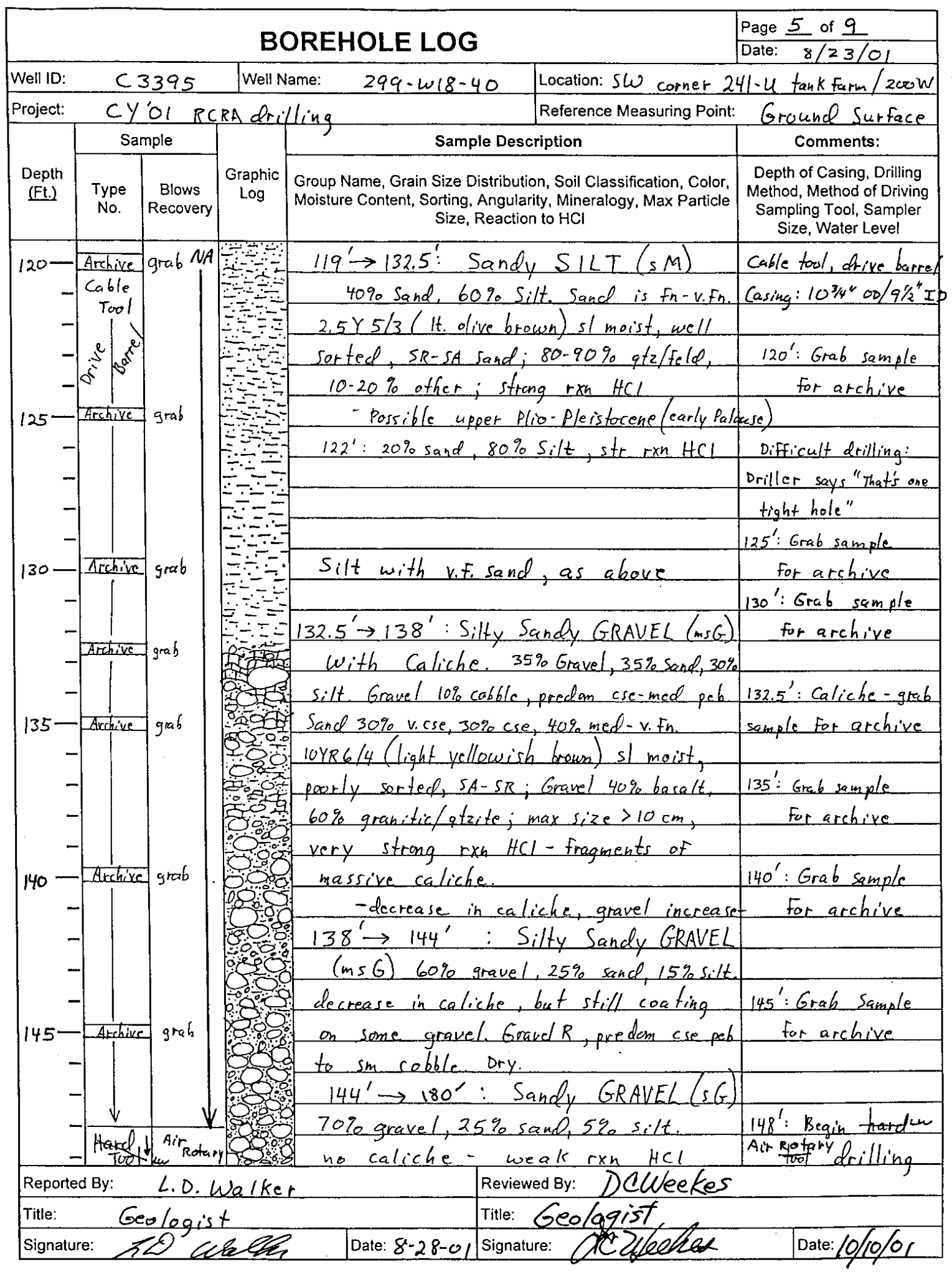

BHI-EE-183 (12/97) 


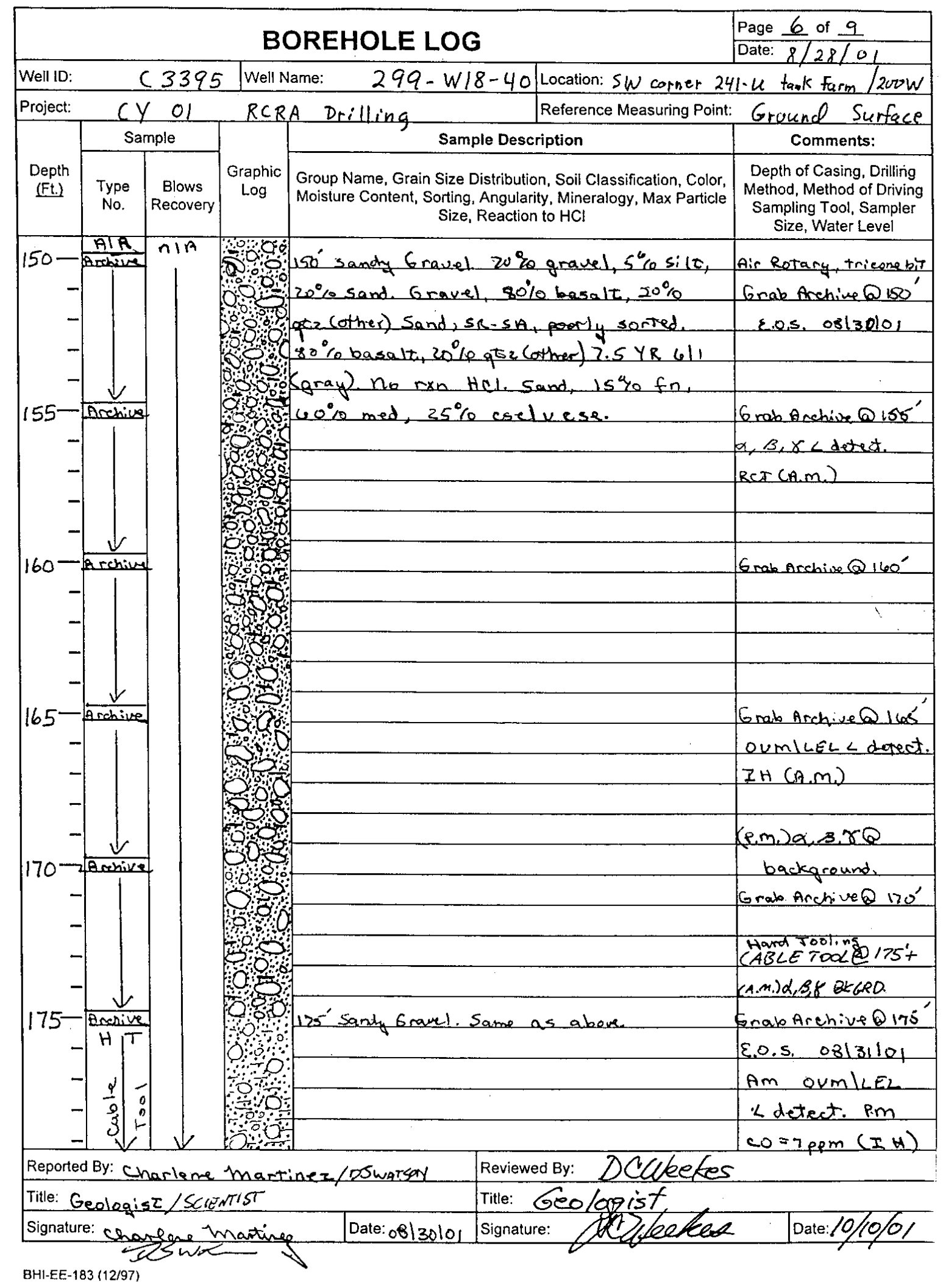




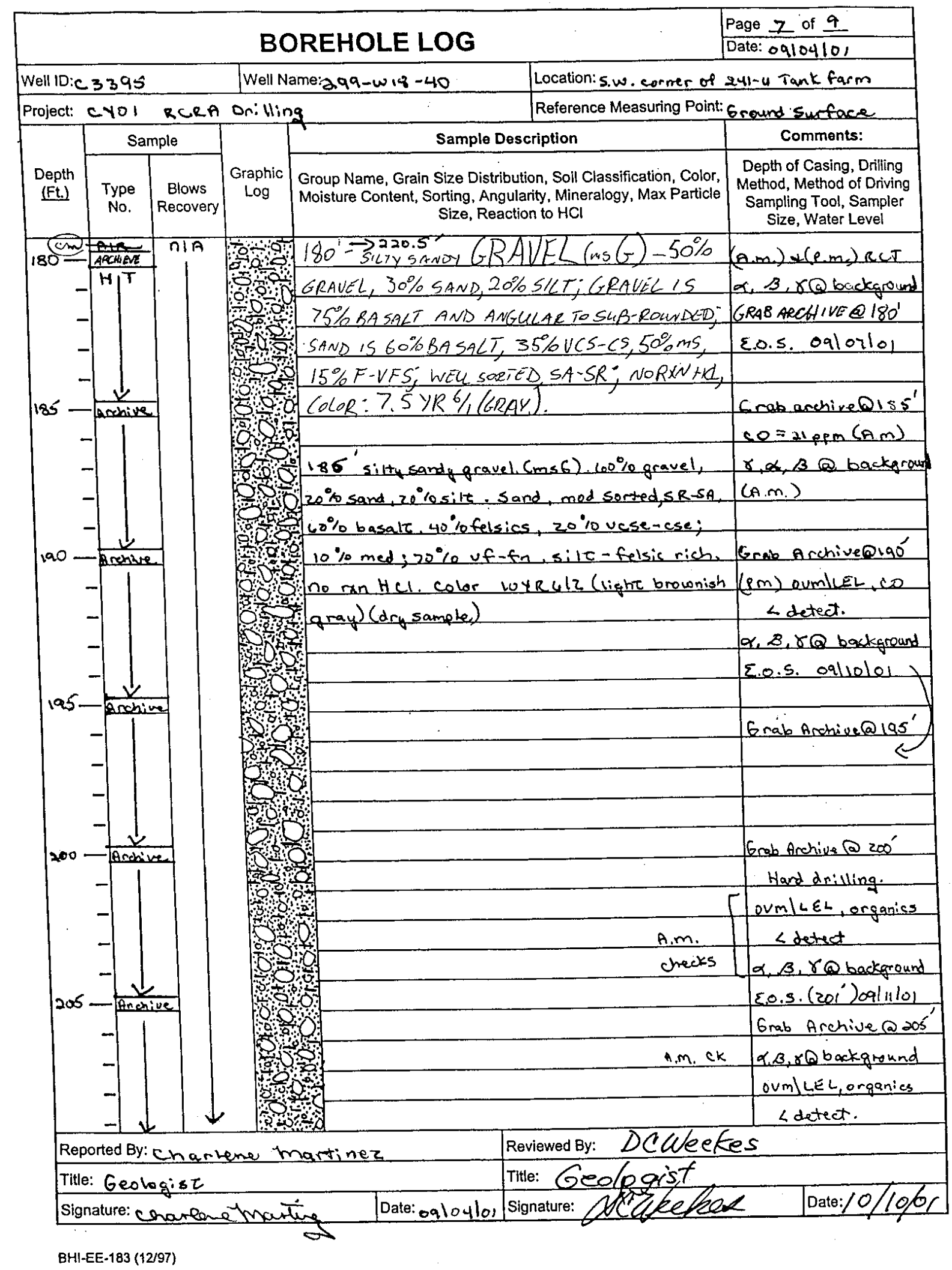




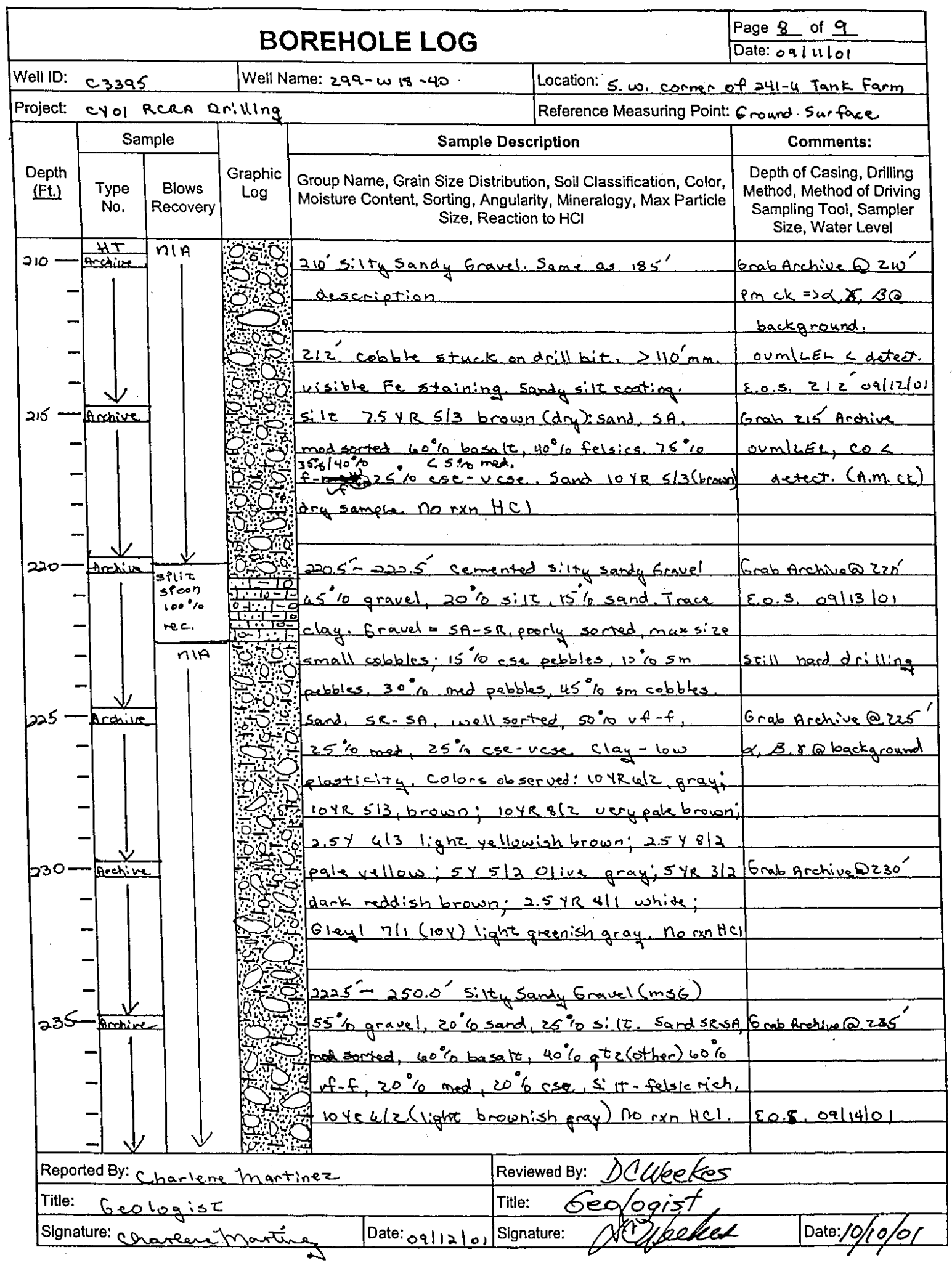

BHI-EE-183 (12/97) 


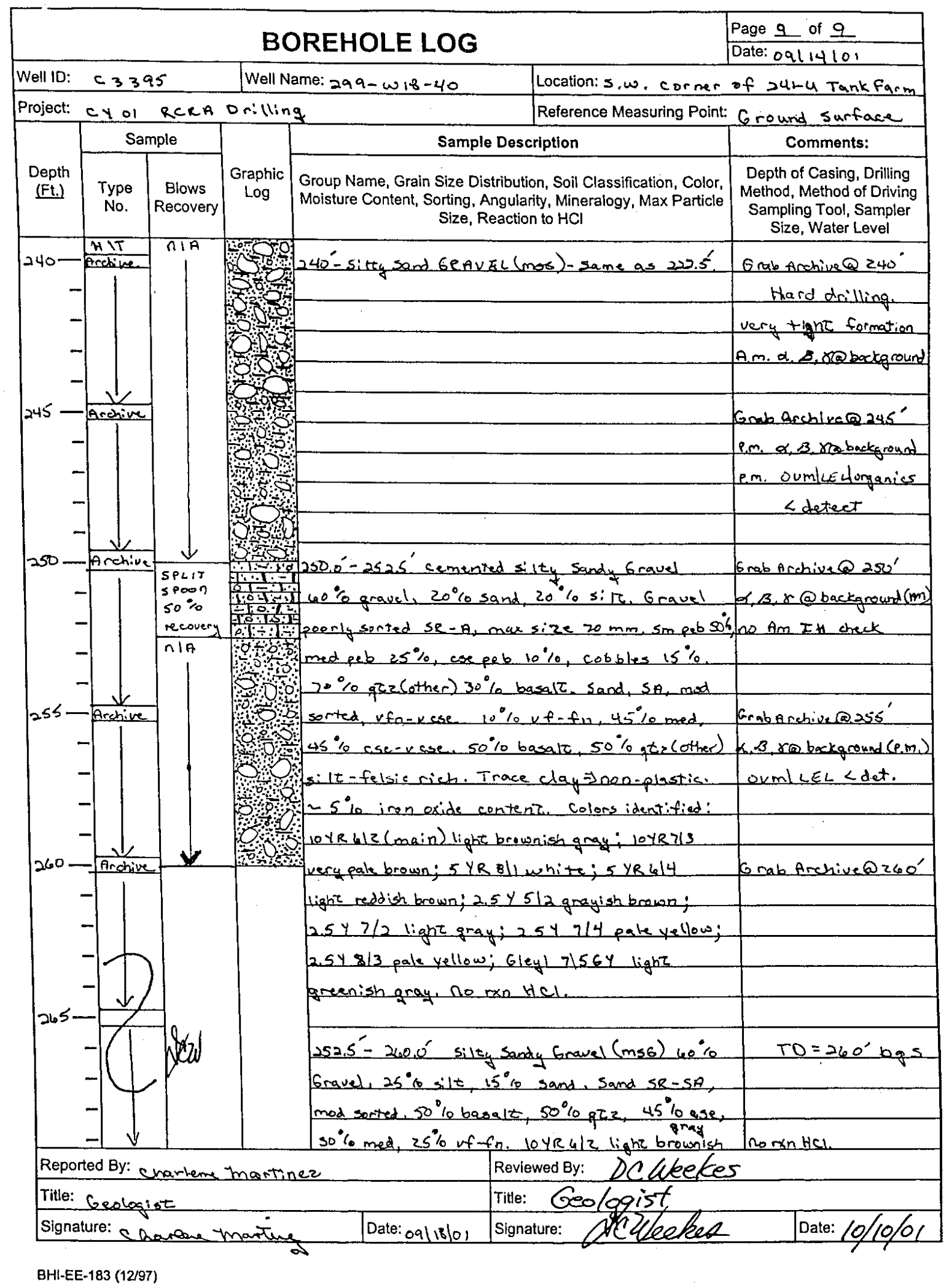




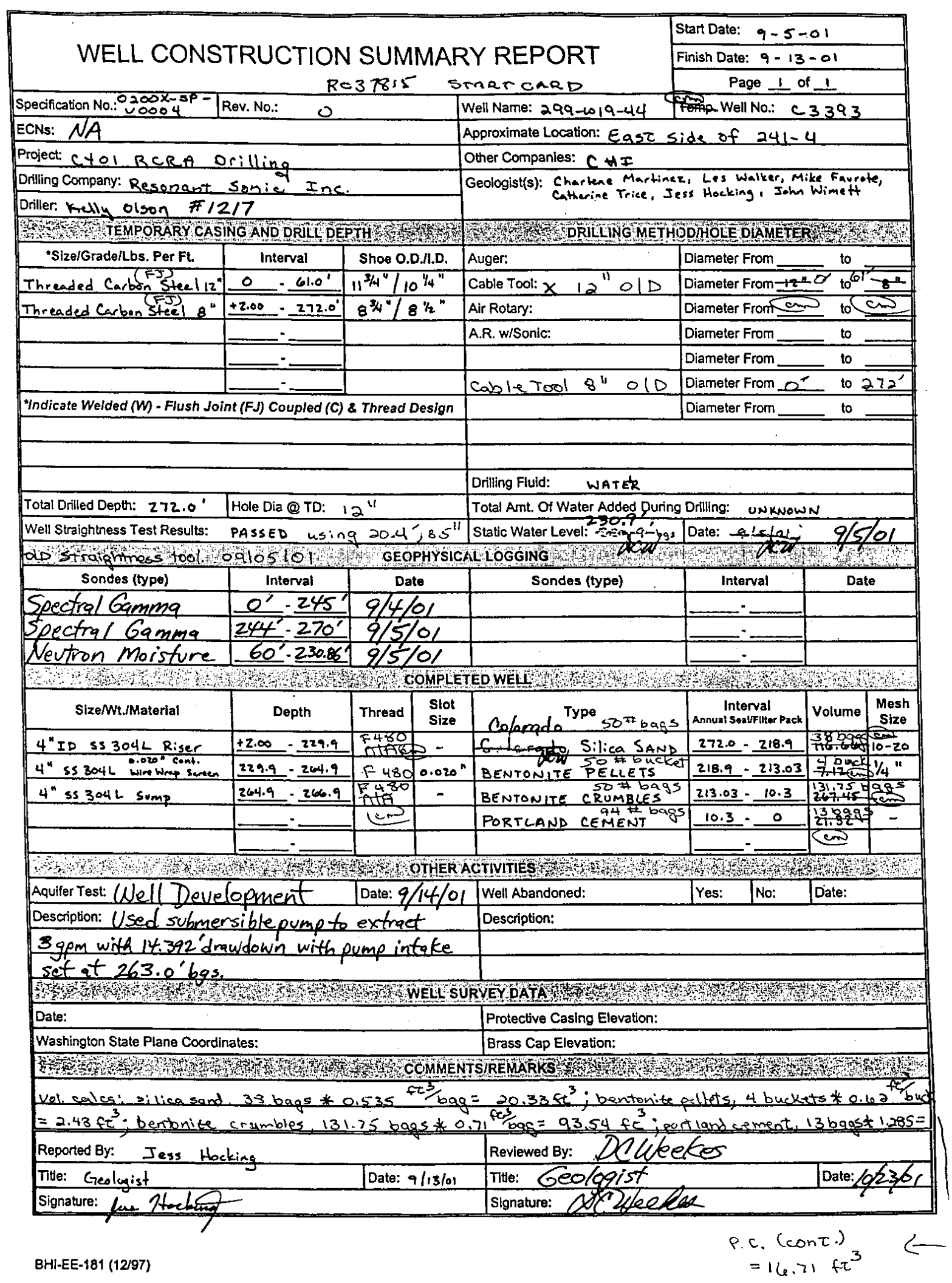




\begin{tabular}{|c|c|c|c|}
\hline \multirow{2}{*}{\multicolumn{4}{|c|}{ WELL SUMMARY SHEET }} \\
\hline & & & Date: $08 \mid 2>101$ \\
\hline \multicolumn{2}{|l|}{ Well ID: C 3393} & \multicolumn{2}{|c|}{ Well Name: $299-\omega_{19} 9-44$} \\
\hline \multicolumn{2}{|c|}{ Location: Easc of $241-4$ Tank Farm } & \multirow{2}{*}{\multicolumn{2}{|c|}{$\begin{array}{l}\text { Project: Cyol RCRA Drilling } \\
\text { Reviewed By: DCleekes }\end{array}$}} \\
\hline Prepared By: C thanimez/Jess Hocking & Date: of 130101 & & \\
\hline \multicolumn{2}{|c|}{ 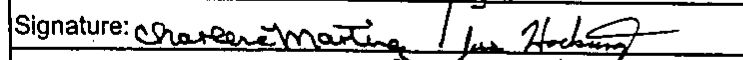 } & \multirow{2}{*}{\multicolumn{2}{|c|}{ Signature: XC Zfeeleses }} \\
\hline \multicolumn{2}{|c|}{ CONSTRUCTIONDATA } & & \\
\hline Description & Diagram & $\begin{array}{c}\text { Depth in } \\
\text { Feet }\end{array}$ & Lithologic Description \\
\hline 6"1D ss 304 Protective casing 3,600 & \multirow{22}{*}{ 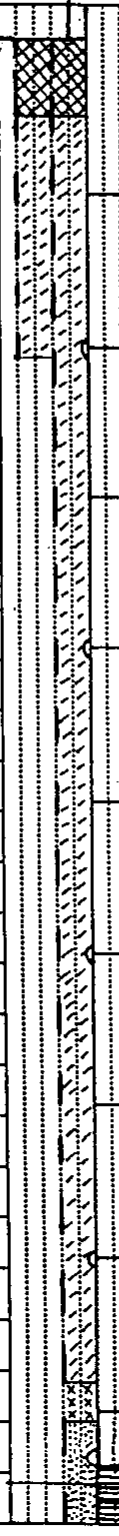 } & \multirow{22}{*}{ 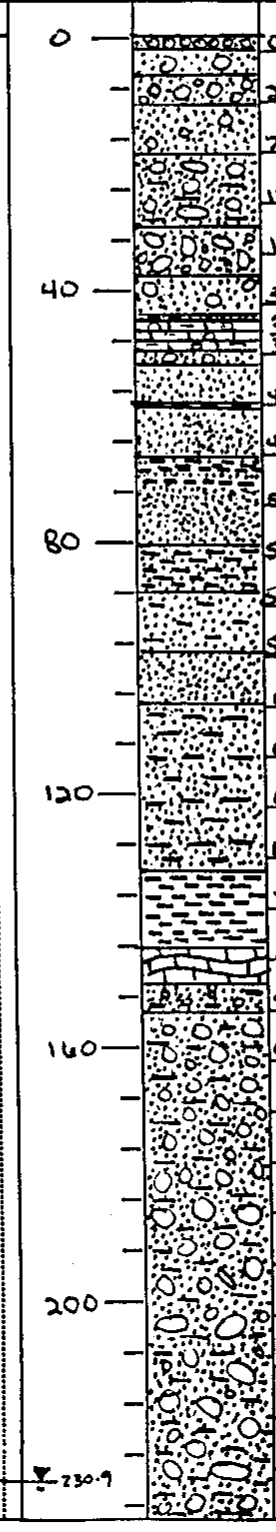 } & \multirow{2}{*}{$\begin{array}{l}0^{\prime}-25^{\prime} \text { sandy gravel }(39) \\
2.5^{\prime}-20^{\prime} \text { gravelly } \operatorname{sand}(0.5)\end{array}$} \\
\hline 4"ID SS 304L RISER & & & \\
\hline$+2.00^{\prime} \rightarrow 229.9^{\prime} \mathrm{bgs.}$ & & & \multirow{6}{*}{ 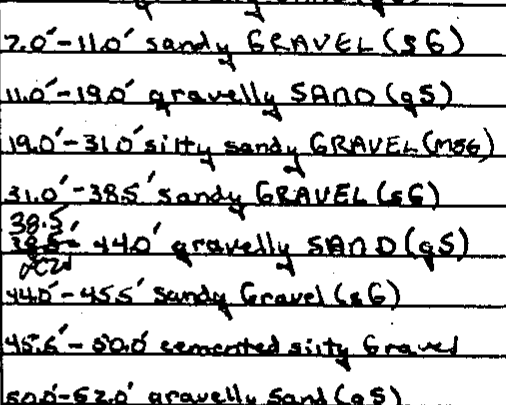 } \\
\hline 4 "I0 ss 304L $0.020 "$ Cont. Wire & & & \\
\hline Wrap Screen. & & & \\
\hline $229.9 \mathrm{bg} \rightarrow 264.9^{\circ} \mathrm{bgs}$ & & & \\
\hline 4"ID S5304L 2ff. SUMP & & & \\
\hline $264.9_{\mathrm{gg}}^{\prime} \rightarrow 266.9_{\mathrm{gg}}^{\prime}$ & & & \\
\hline Colorado Silica Sand 10-20 mest & & & -59s sIIIS \\
\hline $218.9^{\circ} \mathrm{bgs} \rightarrow 272.0^{\circ} \mathrm{bg}$ & & & $592^{\circ}-67.0^{\circ} \operatorname{san} 0(s)$ \\
\hline $1 / 4 "$ Bentonite Pellets & & & \\
\hline $213.03^{\prime} \mathrm{bgs} \rightarrow 218.9^{\prime} \mathrm{bgs}$ & & & $0^{\circ}-690^{\circ} \sin \theta(5)$ \\
\hline Bentonite Crumbles & & & $20.5^{\prime}-81.5^{\prime} \operatorname{so00}(8)$ \\
\hline $10.3^{\prime} \mathrm{bgs} \rightarrow 213.03^{\prime} \mathrm{bgs}$ & & & $815^{\prime}-88.0^{\prime} 50$ \\
\hline Partland Cement Grout & & & \\
\hline $0^{\prime} \rightarrow 10.3^{\prime}$ bgs. & & & $100.0^{\prime}-131.8^{\prime}$ suty sano(ms) \\
\hline & & & $131.8^{\prime}-144.5^{\prime}$ SilT $(m)$ \\
\hline & & & \\
\hline All depths are in fert & & & $155.0^{\prime}-233^{\prime}$ silty sandy GRAver \\
\hline below ground surface & & & \\
\hline ALL TEMP. CASING & & & \\
\hline REMOVED FROM GI & & & \\
\hline
\end{tabular}

BHI-EE-189 (12/97) 


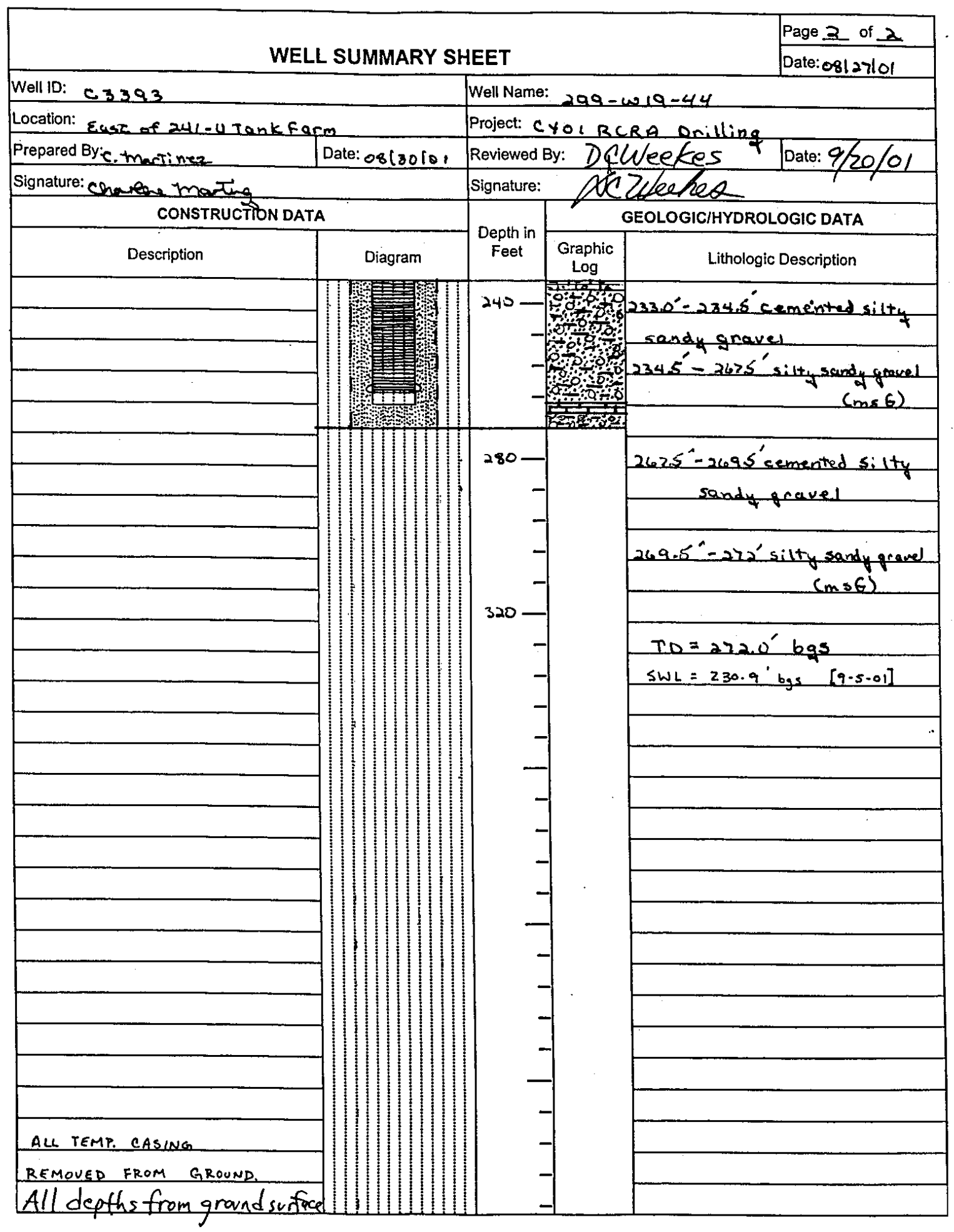

BHI-EE-189 (12/97) 


\begin{tabular}{|c|c|c|c|c|c|c|}
\hline \multicolumn{7}{|c|}{ WELL SURVEY DATA REPORT } \\
\hline \multicolumn{4}{|c|}{ ERC Project: 22192} & \multicolumn{3}{|c|}{$\begin{array}{l}\text { Prepared By: Gary B. Wagner, P.L.S. } \\
\text { Company: Rogers Surveying, Inc. }\end{array}$} \\
\hline \multicolumn{4}{|c|}{ Date Requested $11 / 19 / 01$} & \multicolumn{3}{|c|}{ Requestor: } \\
\hline \multicolumn{4}{|c|}{ Date of Survey: $12 / 05 / 01$} & \multicolumn{3}{|c|}{ Surveyor: Rogers Surveying, Inc. } \\
\hline \multicolumn{4}{|c|}{ ERC Point of Contact: Mr. Robert Bone } & \multicolumn{3}{|c|}{$\begin{array}{l}\text { Survey Co. Point of Contact: } \\
\text { Gary B. Wagner, P.L.S. }\end{array}$} \\
\hline \multirow{4}{*}{\multicolumn{4}{|c|}{$\begin{array}{l}\text { Description of Work: } \\
\text { Civil surveying for eleven groundwater wells } \\
\text { in } 200 \text { W \& } 200 \mathrm{E} \text { Areas. }\end{array}$}} & \multicolumn{3}{|c|}{ Horizontal Datum: NAD83(91) } \\
\hline & & & & \multicolumn{3}{|c|}{ Vertical Datum: NAVD88 } \\
\hline & & & & \multicolumn{3}{|c|}{ Units: Metric } \\
\hline & & & & \multicolumn{3}{|c|}{ Hanford Area Designation: $200 \mathrm{~W}$} \\
\hline \multicolumn{7}{|c|}{ Coordinate System: Washington State Plane Coordinates (South Zone) } \\
\hline \multicolumn{7}{|c|}{$\begin{array}{l}\text { Horizontal Control Monuments: } \\
\text { HSWB-037 \& GPS } 31\end{array}$} \\
\hline \multicolumn{7}{|c|}{$\begin{array}{l}\text { Vertical Control Monuments: } \\
2 W-43 \text { \& HSWB-037 } \\
\end{array}$} \\
\hline Well Name & Well ID & Easting & \multicolumn{2}{|c|}{ Northing } & Elevation & \\
\hline \multirow[t]{3}{*}{$299-W 19.44$} & C3393 & 566896.95 & \multicolumn{2}{|c|}{ 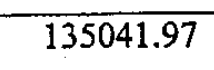 } & & Center of Casing \\
\hline & & & \multirow{2}{*}{\multicolumn{2}{|c|}{135042.26}} & 207.277 & "X" on Rim \\
\hline & & 566896.95 & & & 206.520 & Brass Cap \\
\hline \multicolumn{7}{|l|}{ Notes: } \\
\hline \multicolumn{4}{|c|}{$\begin{array}{l}\text { Surveyor Statement: } \\
\text { 1. Gary B. Wagner, a professional land surveyor } \\
\text { registered in the state of Washington (Registration } \\
\text { No. } 30440 \text { ), hereby certify that this report is based } \\
\text { on a field survey performed in December, } 2001 \\
\text { under my direct supervision and that the data } \\
\text { contained here is true and correct. }\end{array}$} & \multicolumn{3}{|c|}{ Certification Seal } \\
\hline
\end{tabular}

BHI-EE-202 (09/98). 


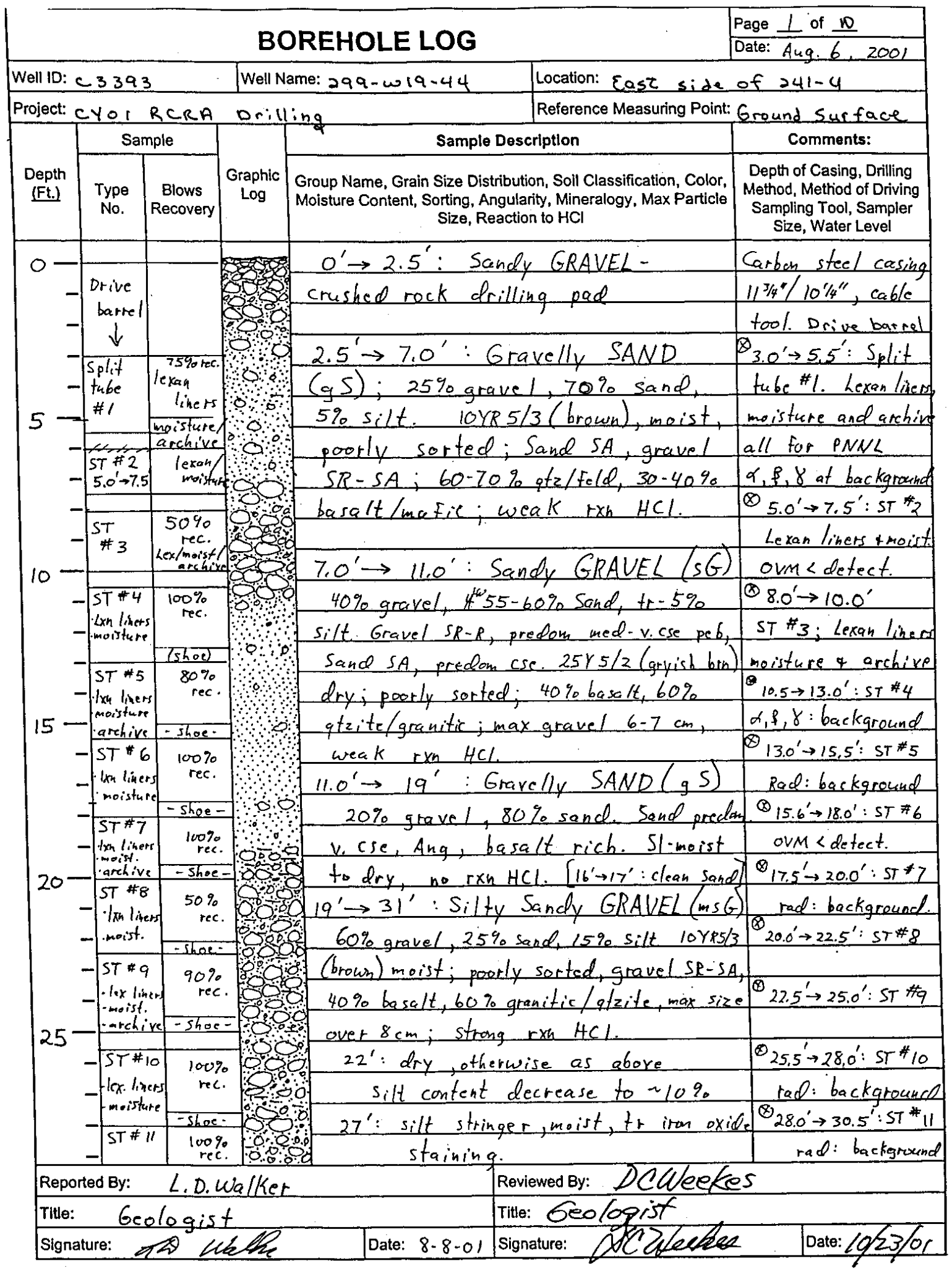

BHI-EE-183 (12/97) 


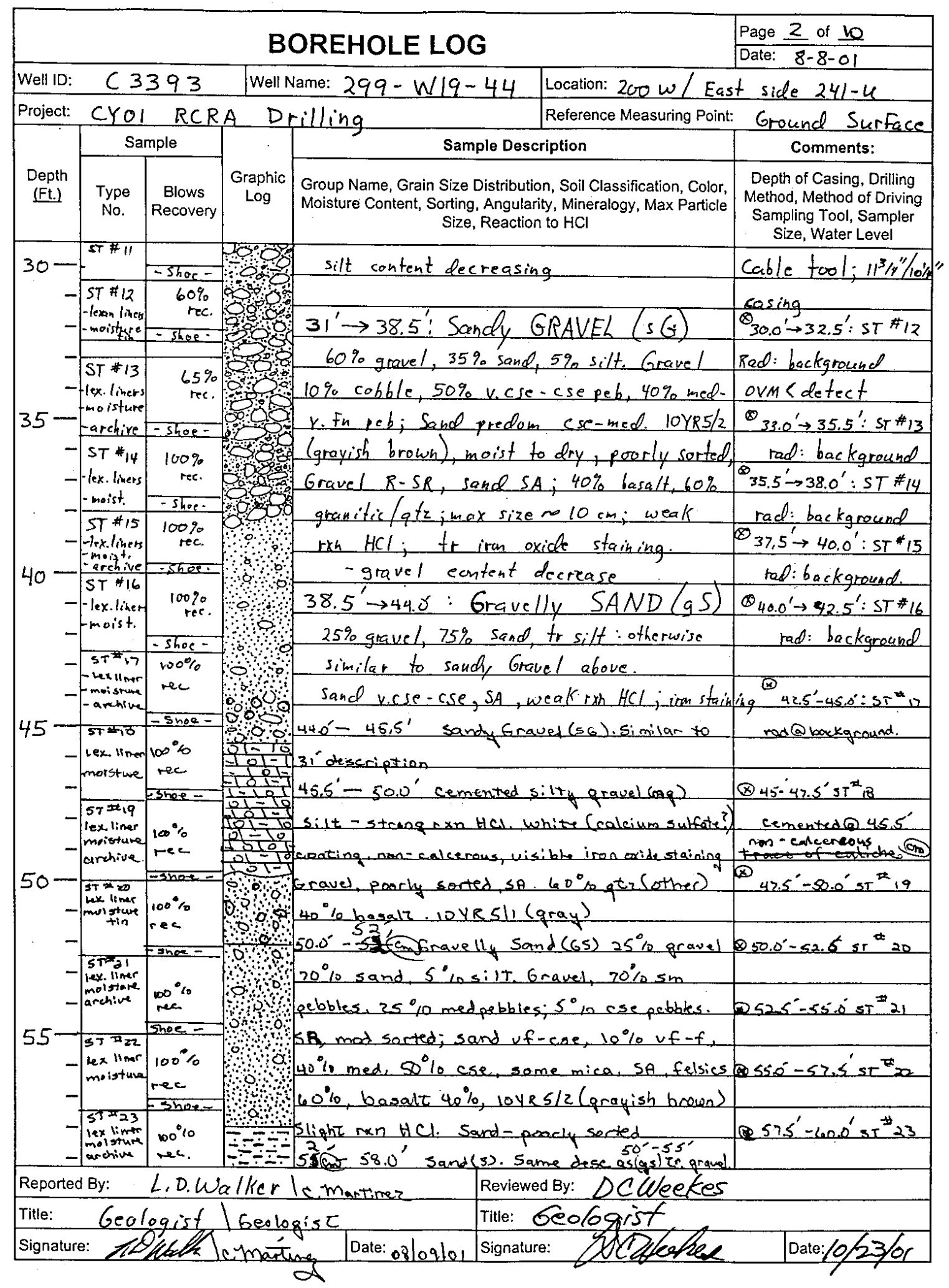




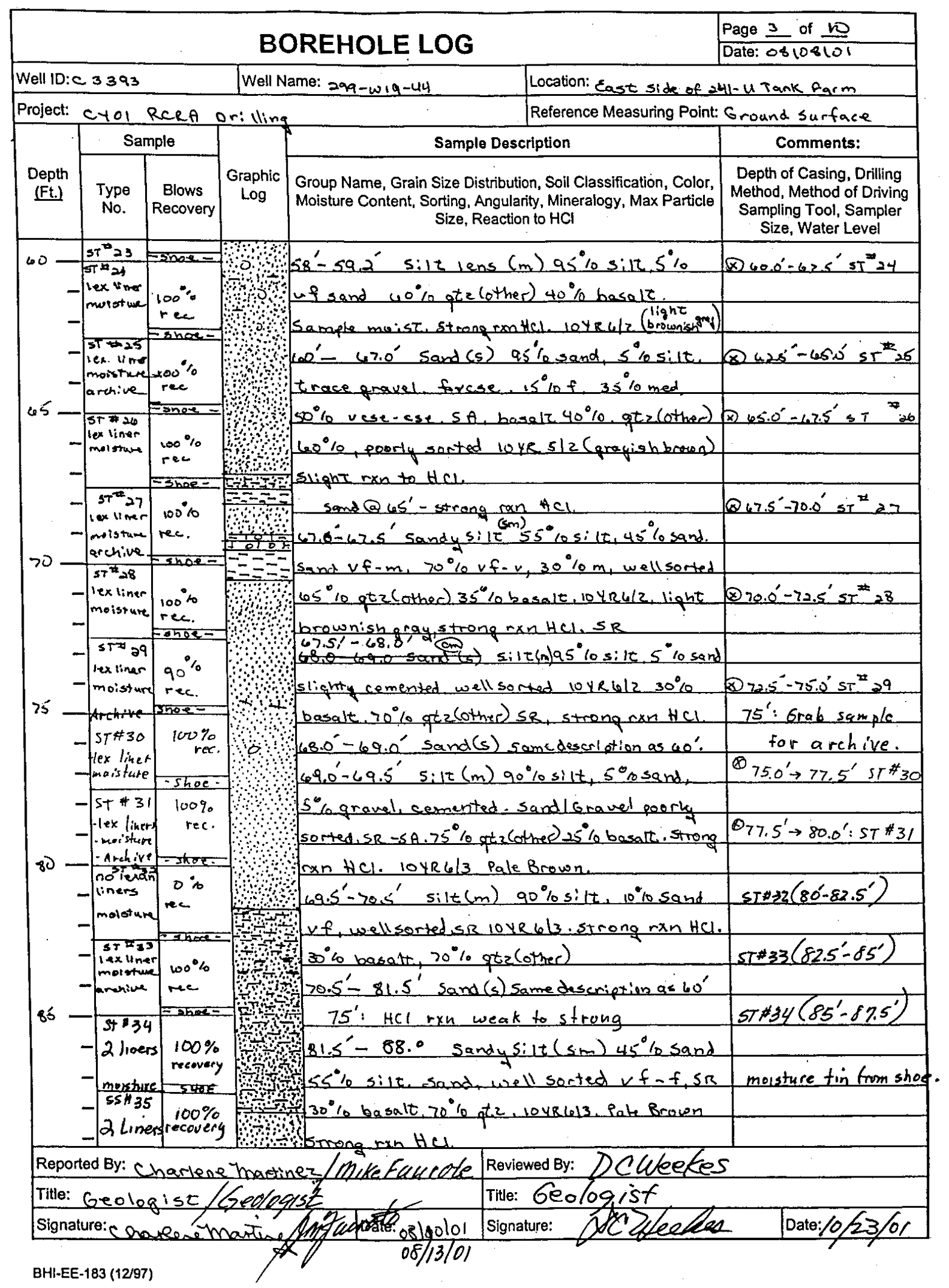




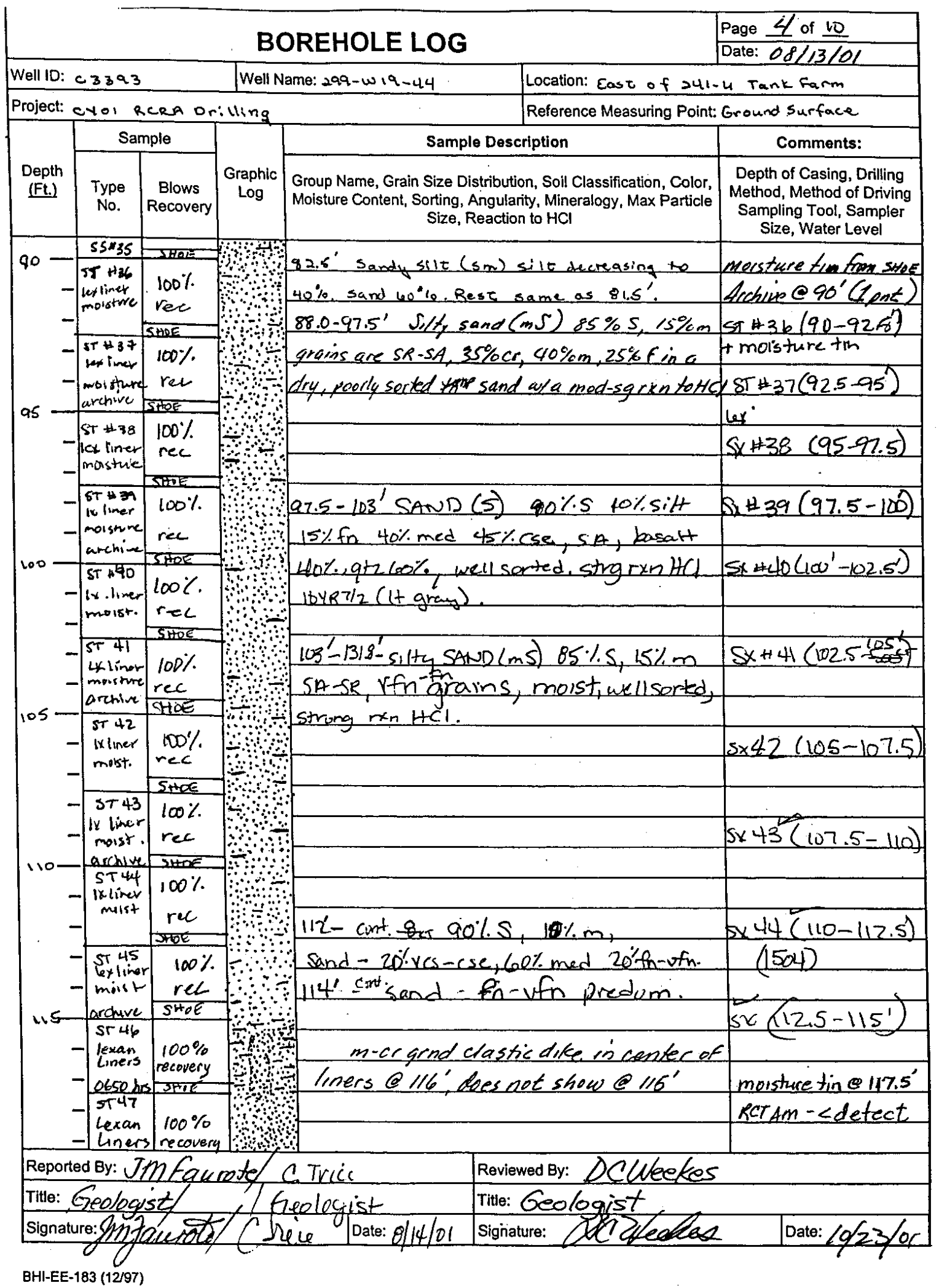




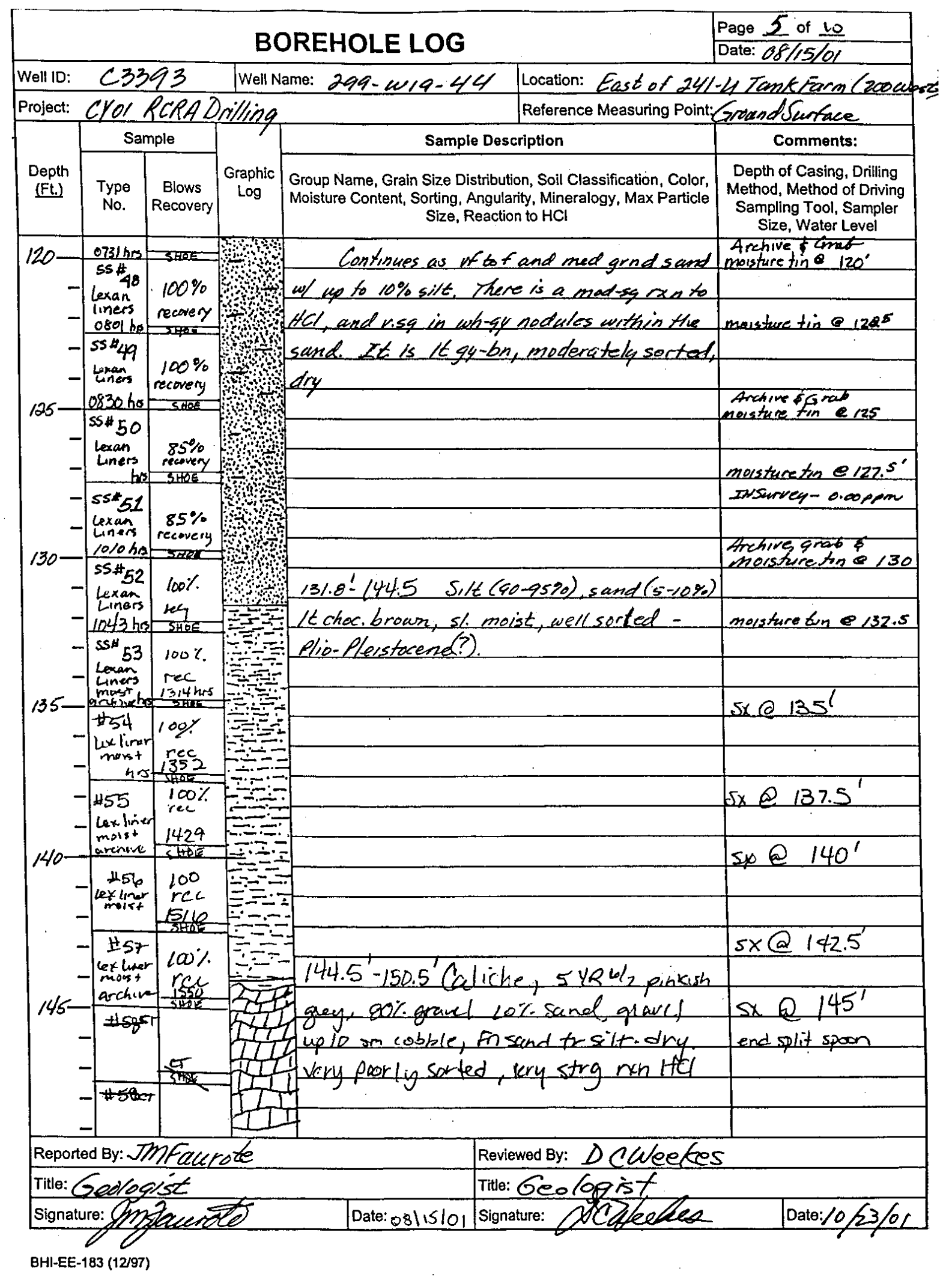




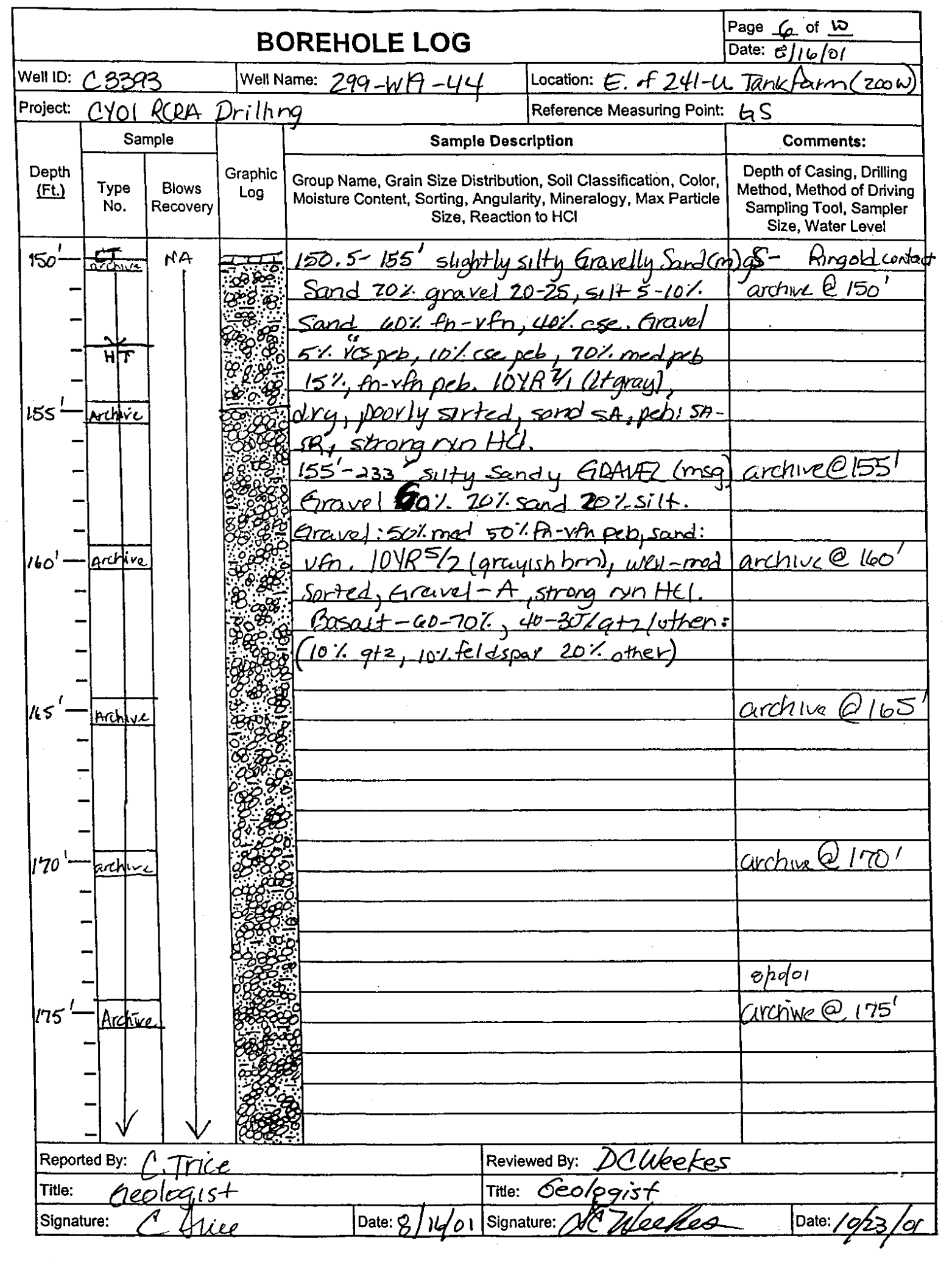

BHI-EE-183 (12/97) 


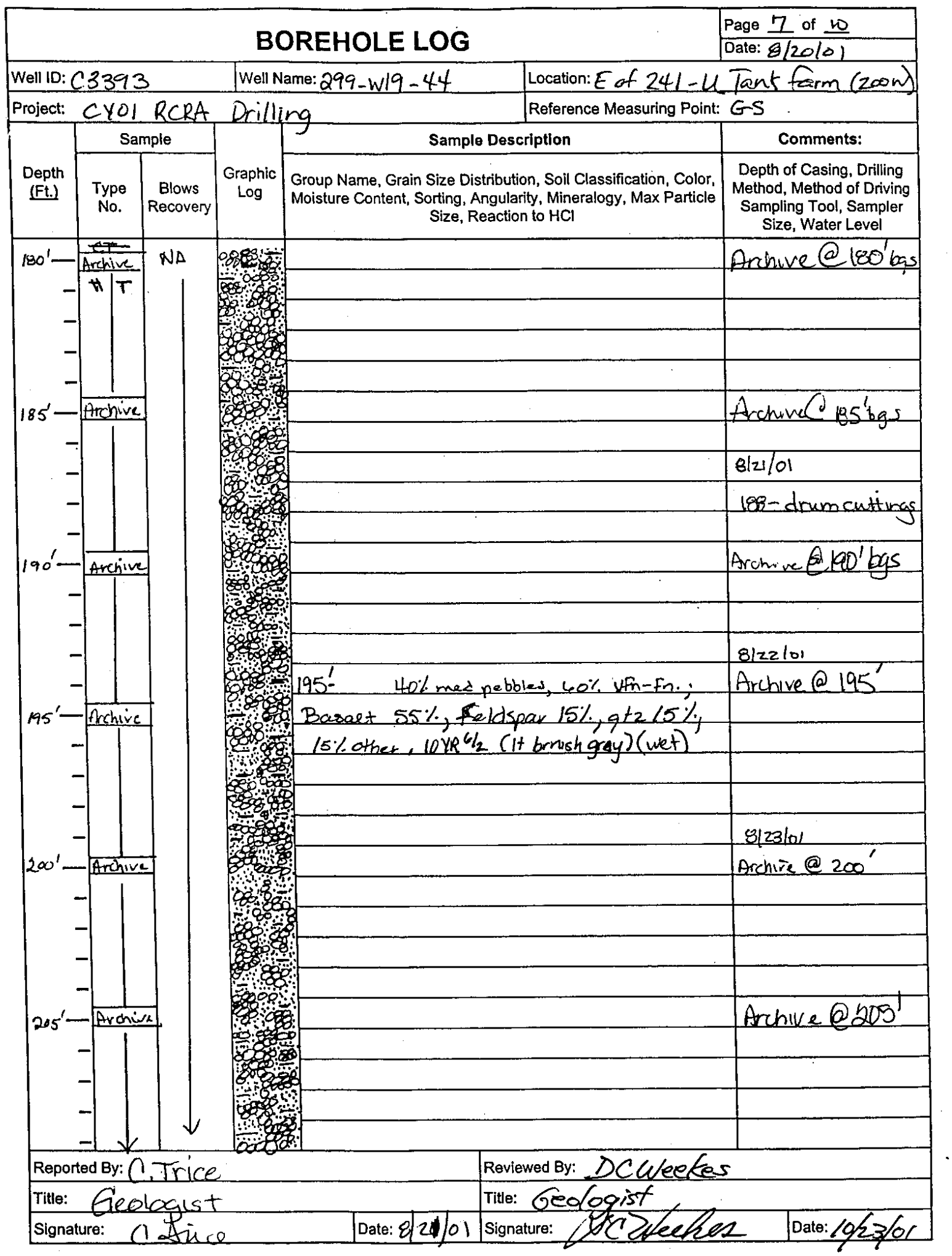

BHI-EE-183 (12/97) 


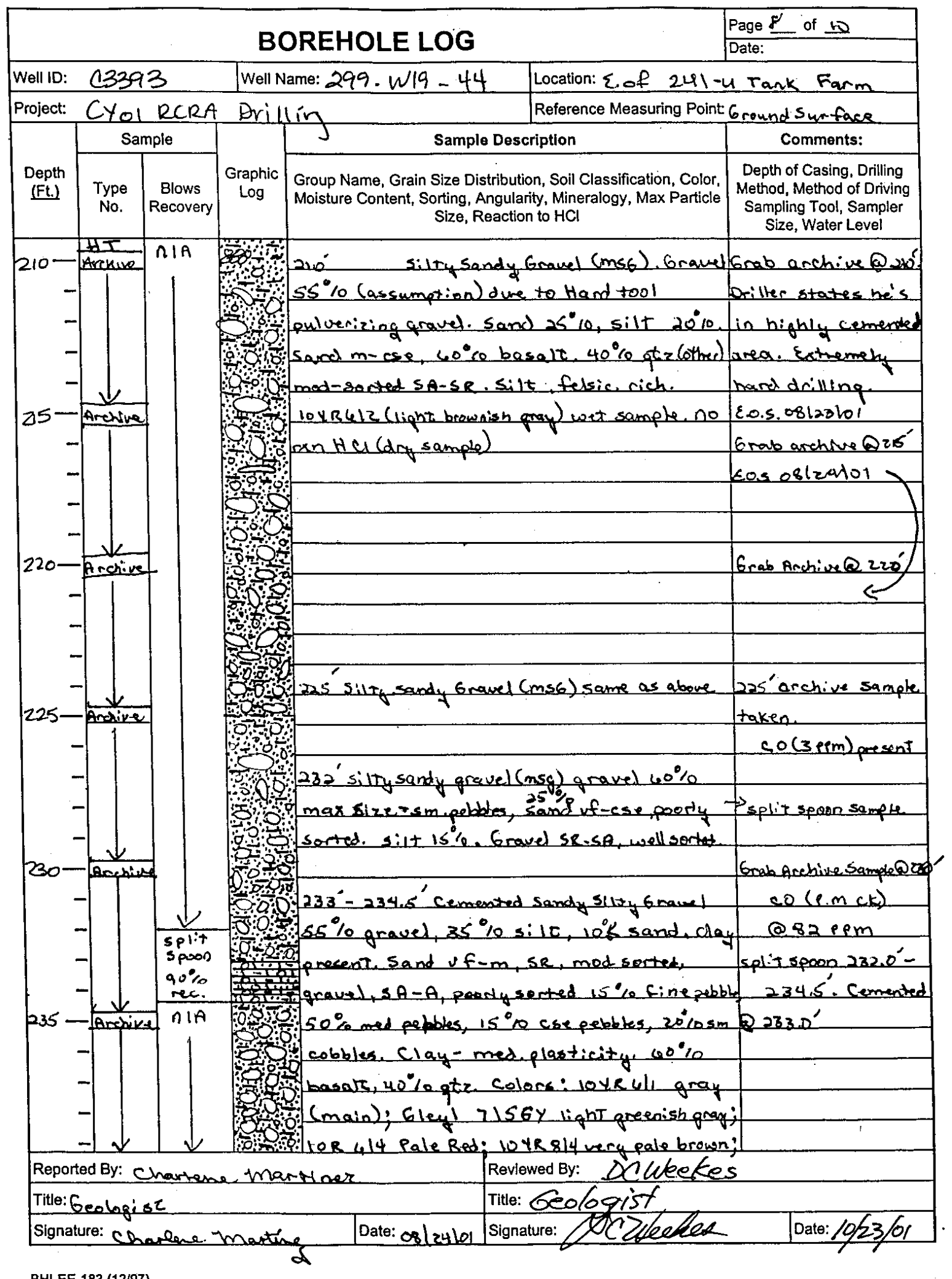




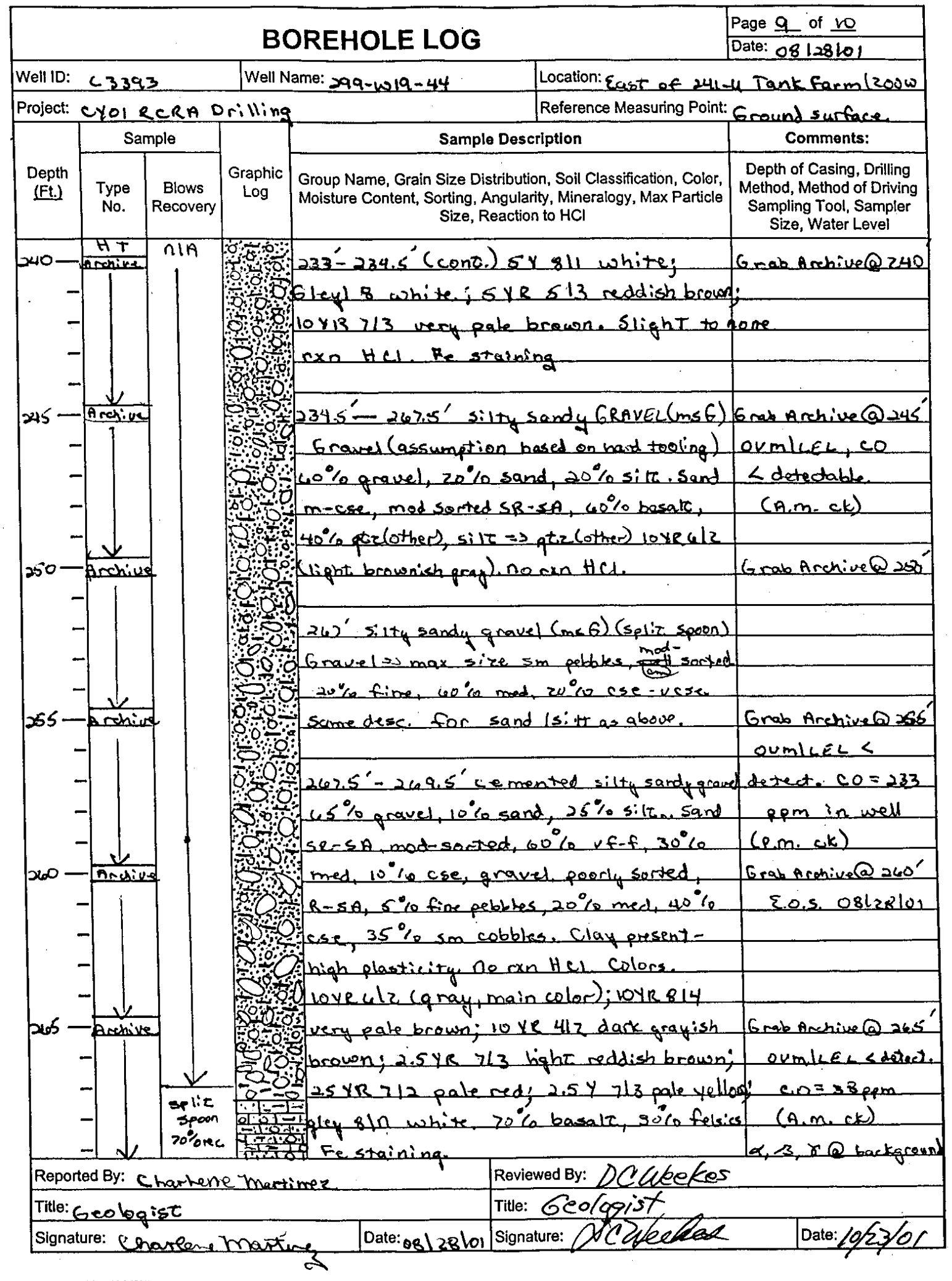

BHI-EE-183 (12/97) 


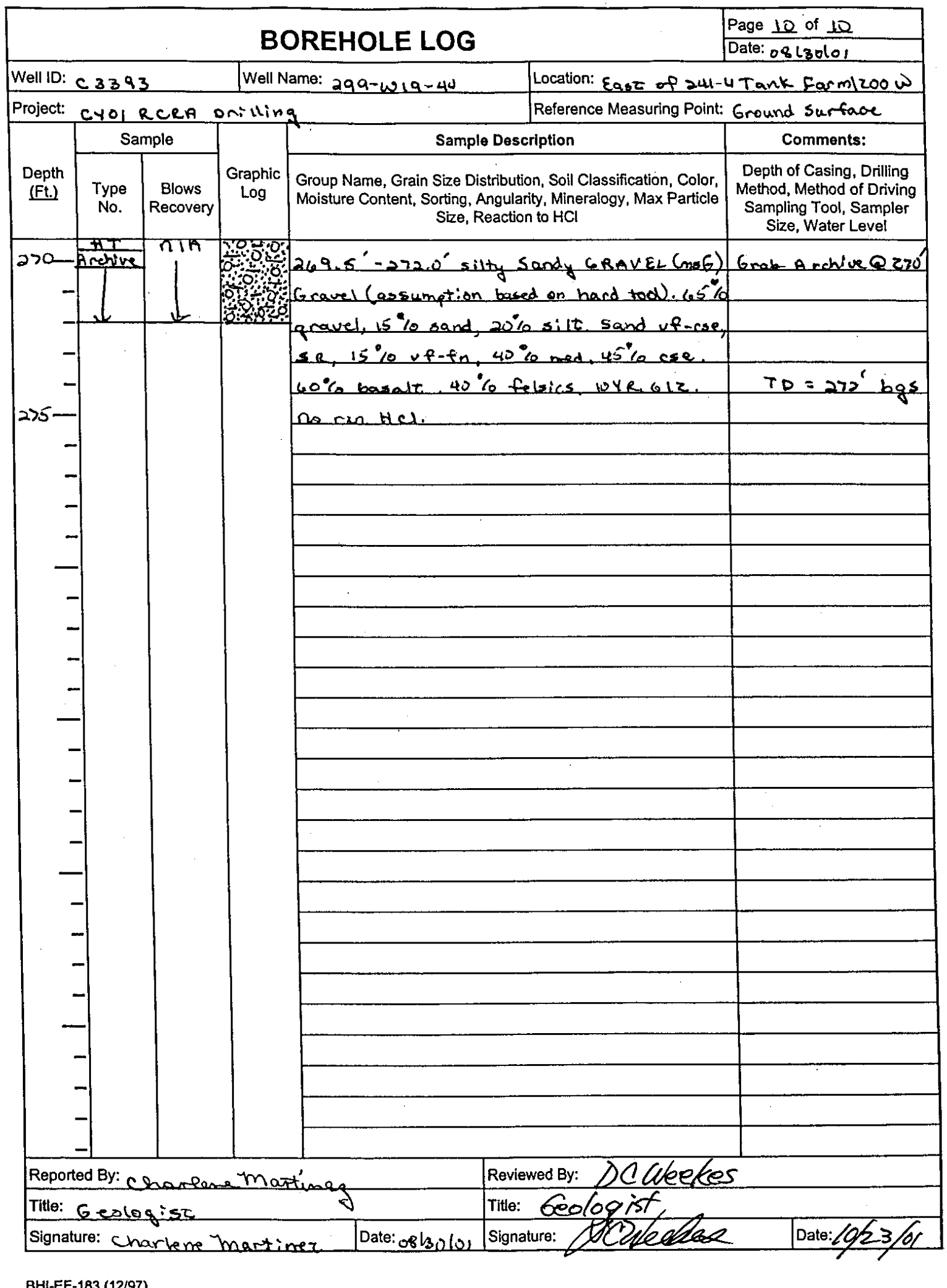

BHI-EE-183 (12/97) 


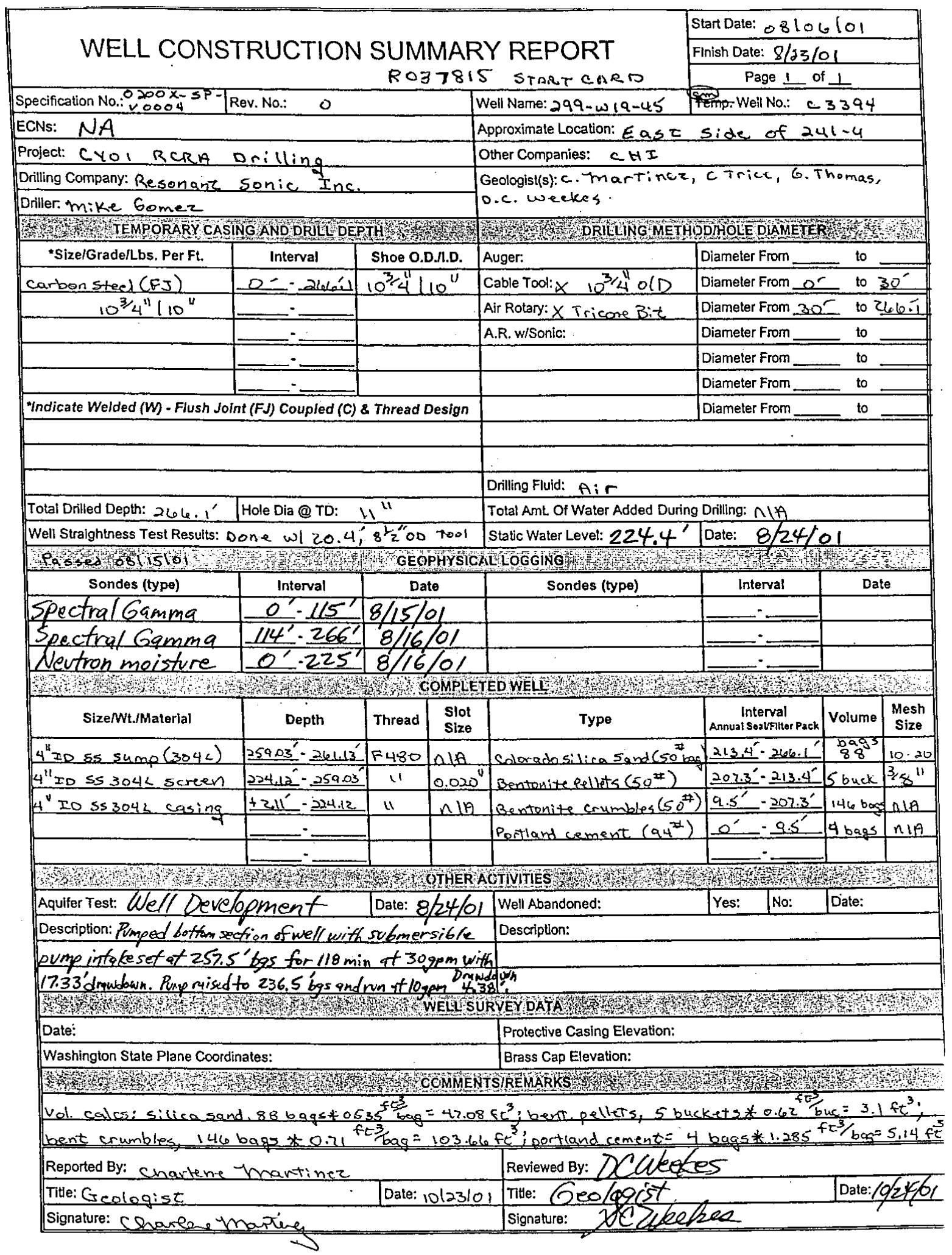

BHI-EE-181 (12/97) 


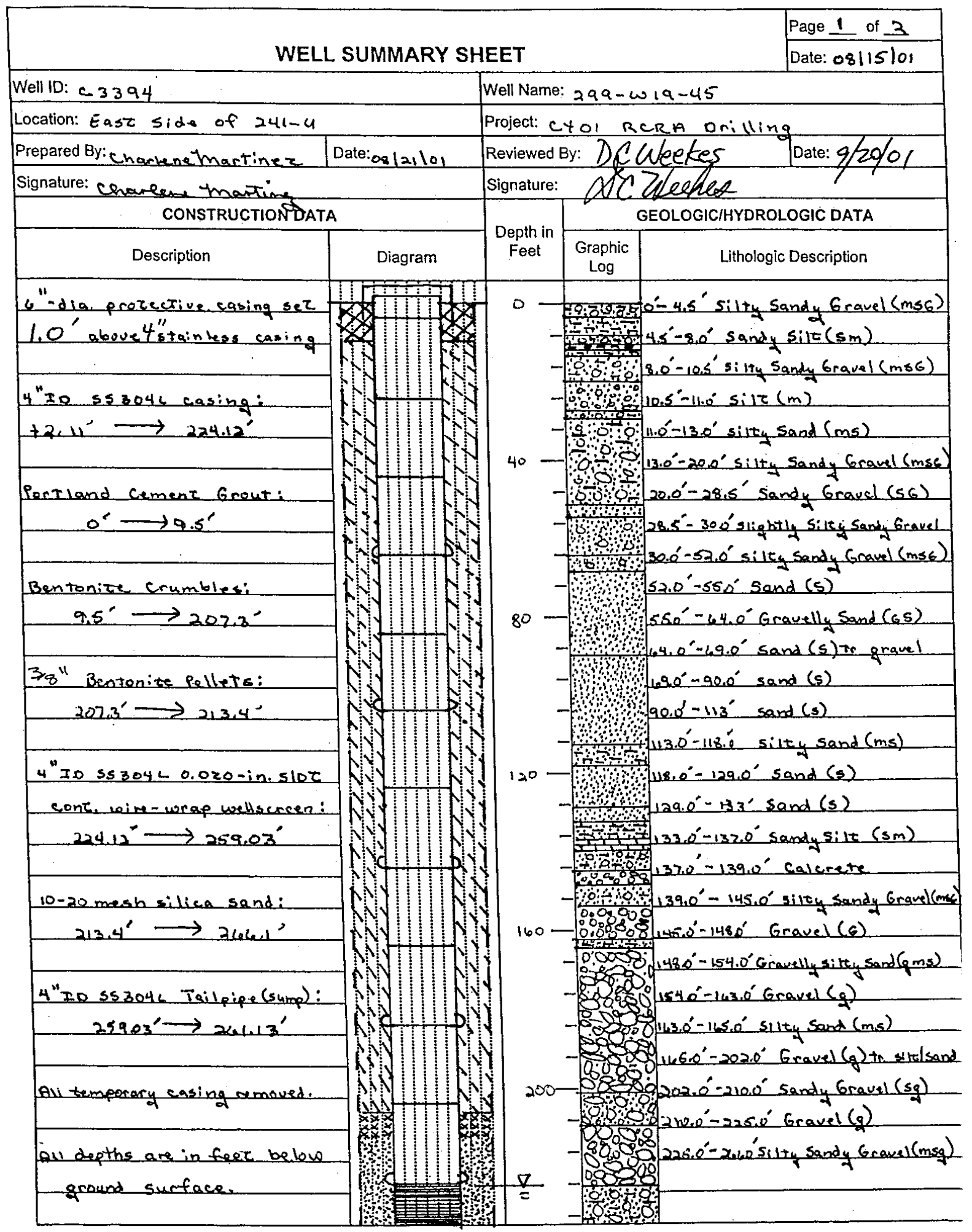

BHI-EE-189 (12/97) 


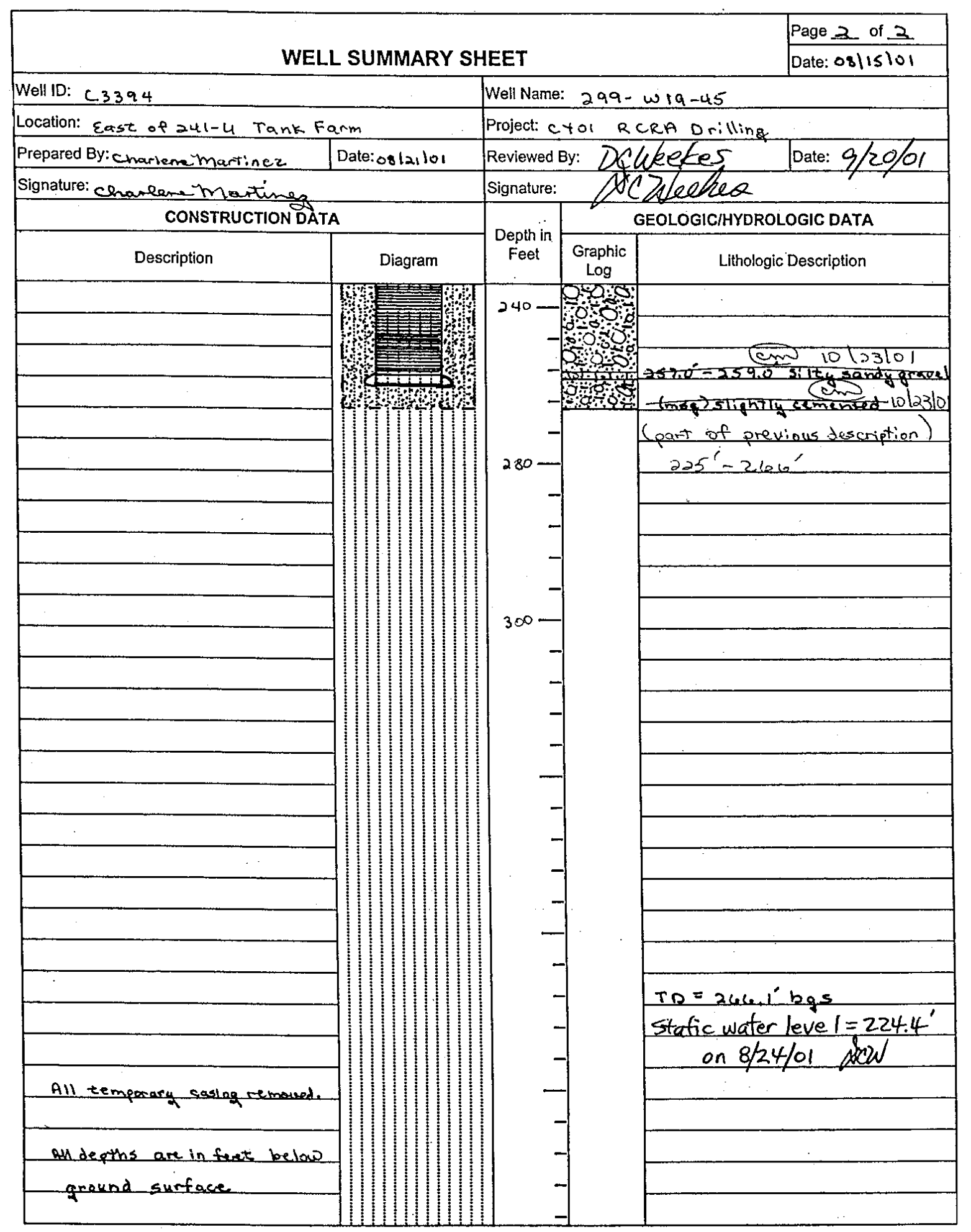

BHI-EE-189 (12/97) 


\begin{tabular}{|c|c|c|c|c|c|c|}
\hline \multicolumn{7}{|c|}{ WELL SURVEY DATA REPORT } \\
\hline \multicolumn{4}{|c|}{ ERC Project: 22192} & \multicolumn{3}{|c|}{$\begin{array}{l}\text { Prepared By: Gary B. Wagner, P.L.S. } \\
\text { Company: Rogers Surveying, Inc. }\end{array}$} \\
\hline \multicolumn{4}{|c|}{ Date Requested $11 / 19 / 01$} & \multicolumn{3}{|c|}{ Requestor: } \\
\hline \multicolumn{4}{|c|}{ Date of Survey: $12 / 05 / 01$} & \multicolumn{3}{|c|}{ Surveyor: Rogers Surveying, Inc. } \\
\hline \multicolumn{4}{|c|}{ ERC Point of Contact: Mr. Robert Bone } & \multicolumn{3}{|c|}{$\begin{array}{l}\text { Survey Co. Point of Contact: } \\
\text { Gary B. Wagner, P.L,S. }\end{array}$} \\
\hline \multirow{4}{*}{\multicolumn{4}{|c|}{$\begin{array}{l}\text { Description of Work: } \\
\text { Civil surveying for eleven groundwater wells } \\
\text { in } 200 \mathrm{~W} \& 200 \mathrm{E} \text { Areas. }\end{array}$}} & \multicolumn{3}{|c|}{ Horizontal Datum: NAD83(91) } \\
\hline & & & & \multicolumn{3}{|c|}{ Vertical Datum: NAVD88 } \\
\hline & & & & \multicolumn{3}{|c|}{ Units: Metric } \\
\hline & & & & \multicolumn{3}{|c|}{ Hanford Area Designation: $200 \bar{W}$} \\
\hline \multicolumn{7}{|c|}{ Coordinate System: Washington State Plane Coordinates (South Zone) } \\
\hline \multicolumn{7}{|c|}{$\begin{array}{l}\text { Horizontal Control Monuments: } \\
\text { HSWB-037 \& GPS } 31\end{array}$} \\
\hline \multicolumn{7}{|c|}{$\begin{array}{l}\text { Vertical Control Monuments: } \\
\text { 2W-43 \& HSWB- } 037\end{array}$} \\
\hline Well Name & Well ID & Easting & \multicolumn{2}{|c|}{ Northing } & Elevation & \\
\hline \multirow[t]{2}{*}{$299-W 19-45$} & $\mathrm{C} 3394$ & 566897.65 & \multicolumn{2}{|c|}{135087.65} & & Center of Casing \\
\hline & & & \multirow{2}{*}{\multicolumn{2}{|c|}{135087.88}} & 206.413 & "X" on Rim \\
\hline & & 566897.64 & & & 205.661 & Brass Cap \\
\hline \multicolumn{7}{|l|}{ Notes: } \\
\hline \multicolumn{4}{|c|}{$\begin{array}{l}\text { Surveyor Statement: } \\
\text { 1, Gary B. Wagner, a professional land surveyor } \\
\text { registered in the state of Washington (Registration } \\
\text { No. } 30440 \text { ), hereby certify that this report is based } \\
\text { on a field survey performed in December, } 2001 \\
\text { under my direct supervision and that the data } \\
\text { contained here is itrue and correct. }\end{array}$} & \multicolumn{3}{|c|}{ Certification Seal } \\
\hline
\end{tabular}

BHI-EE-202 (09/98) 


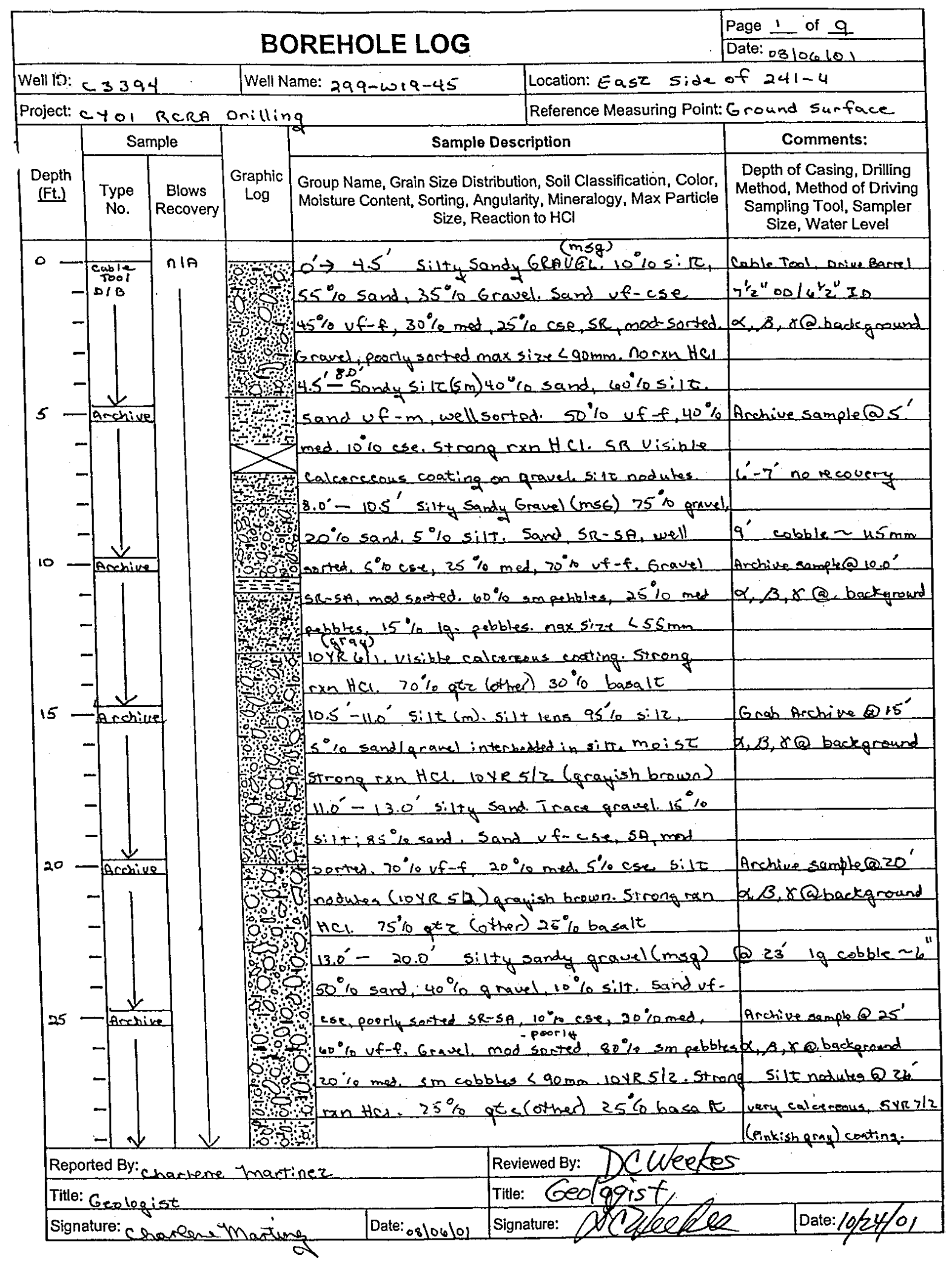

BHI-EE-183 (12/97) 


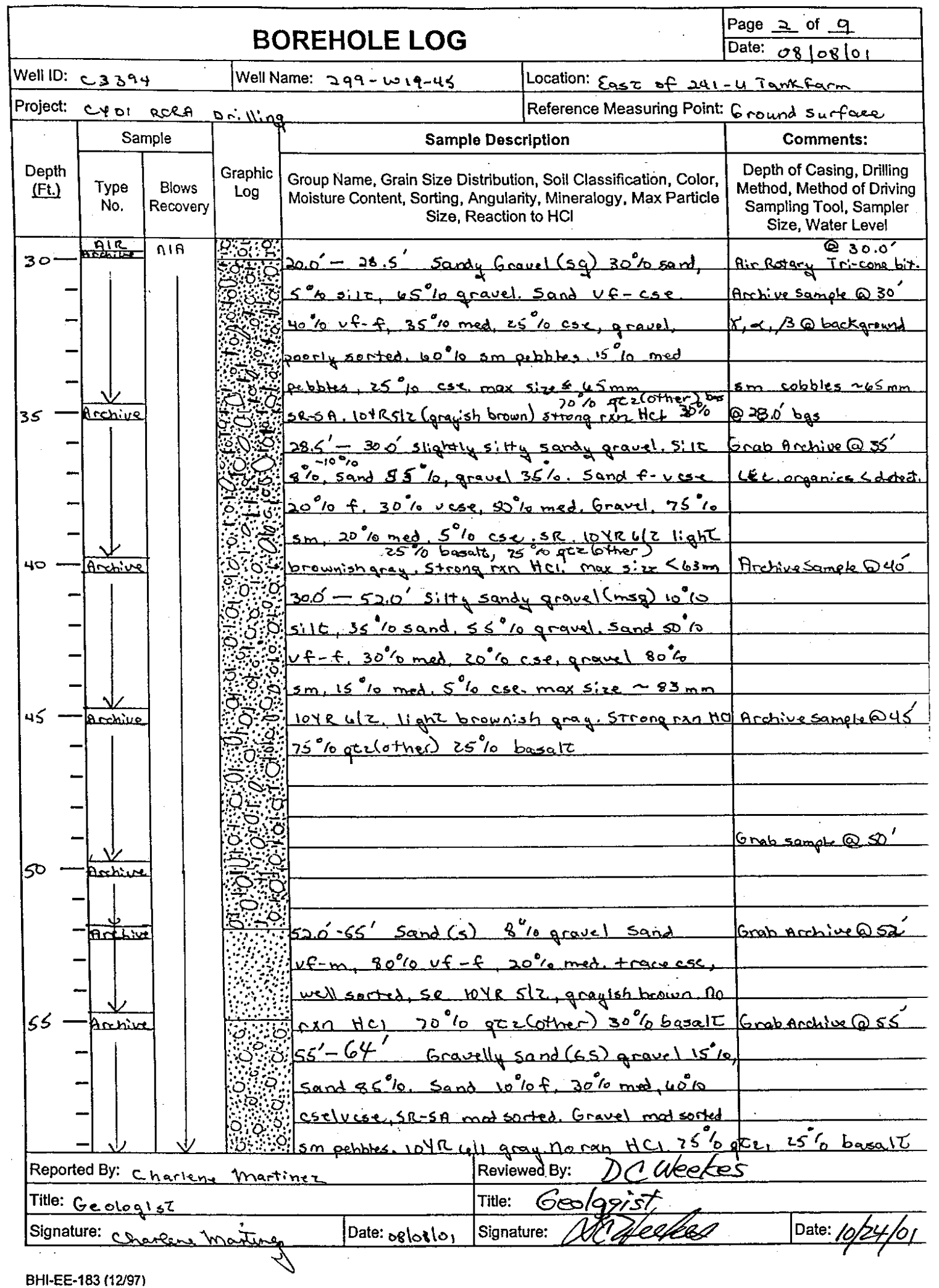




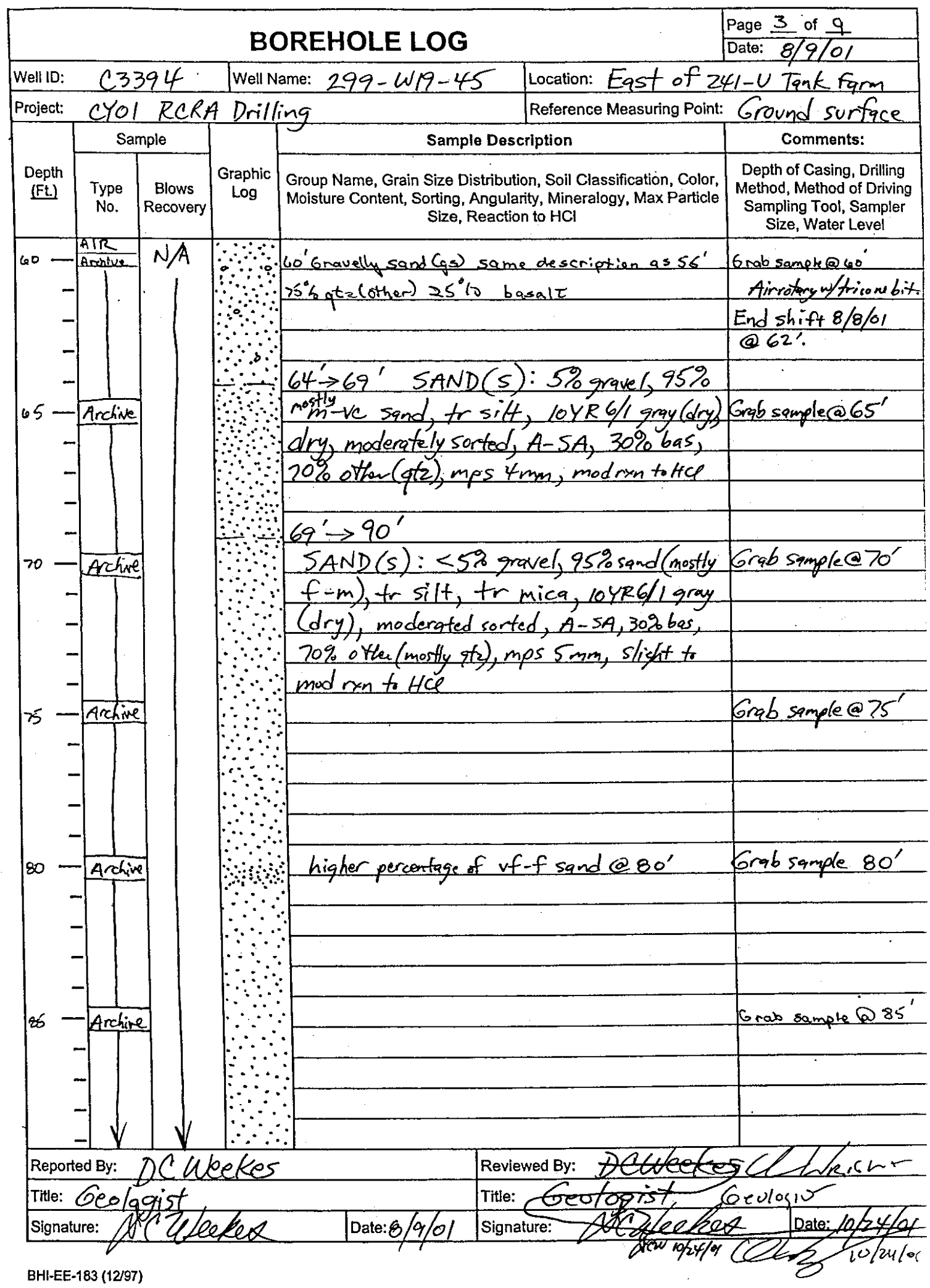




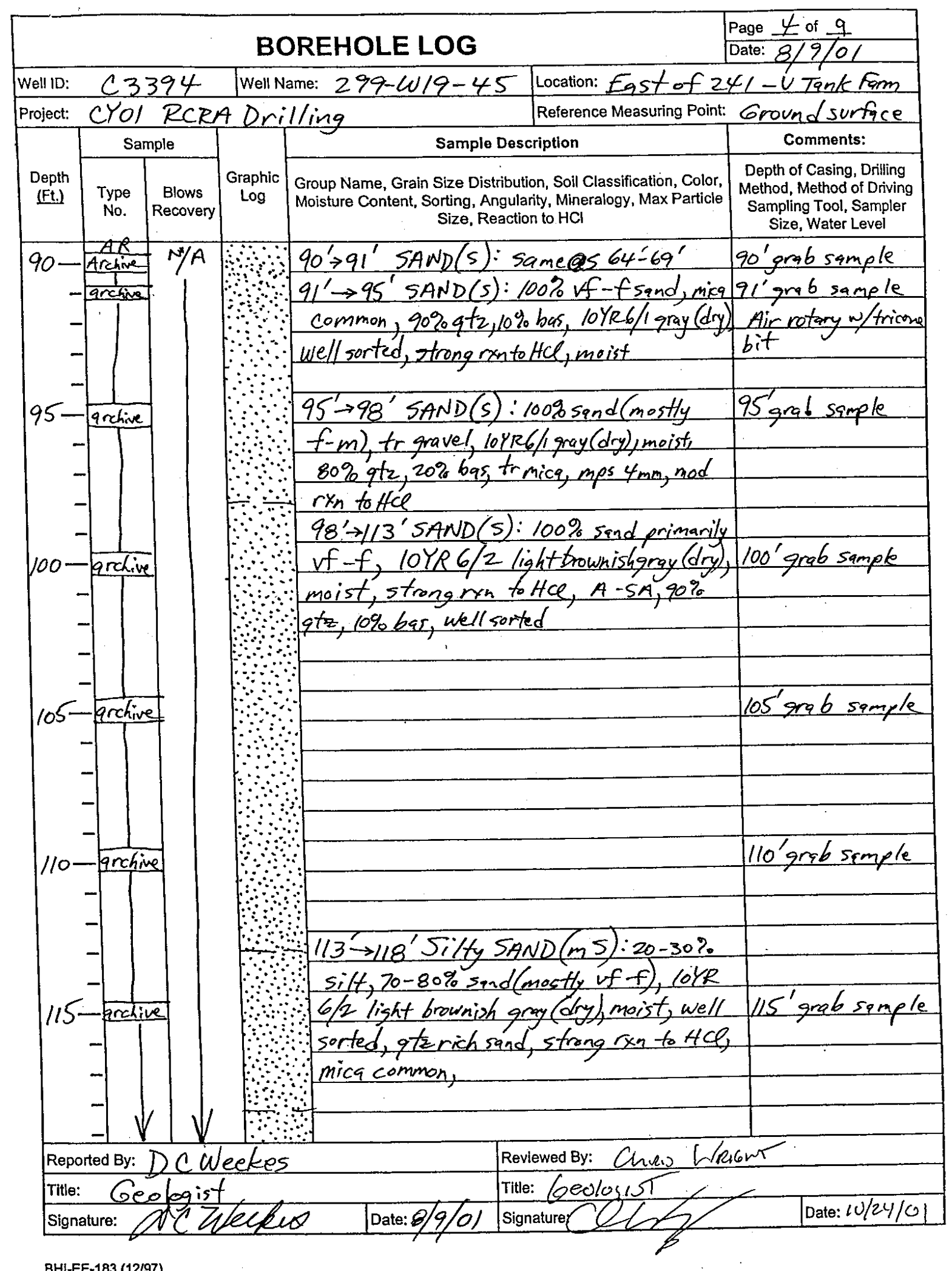




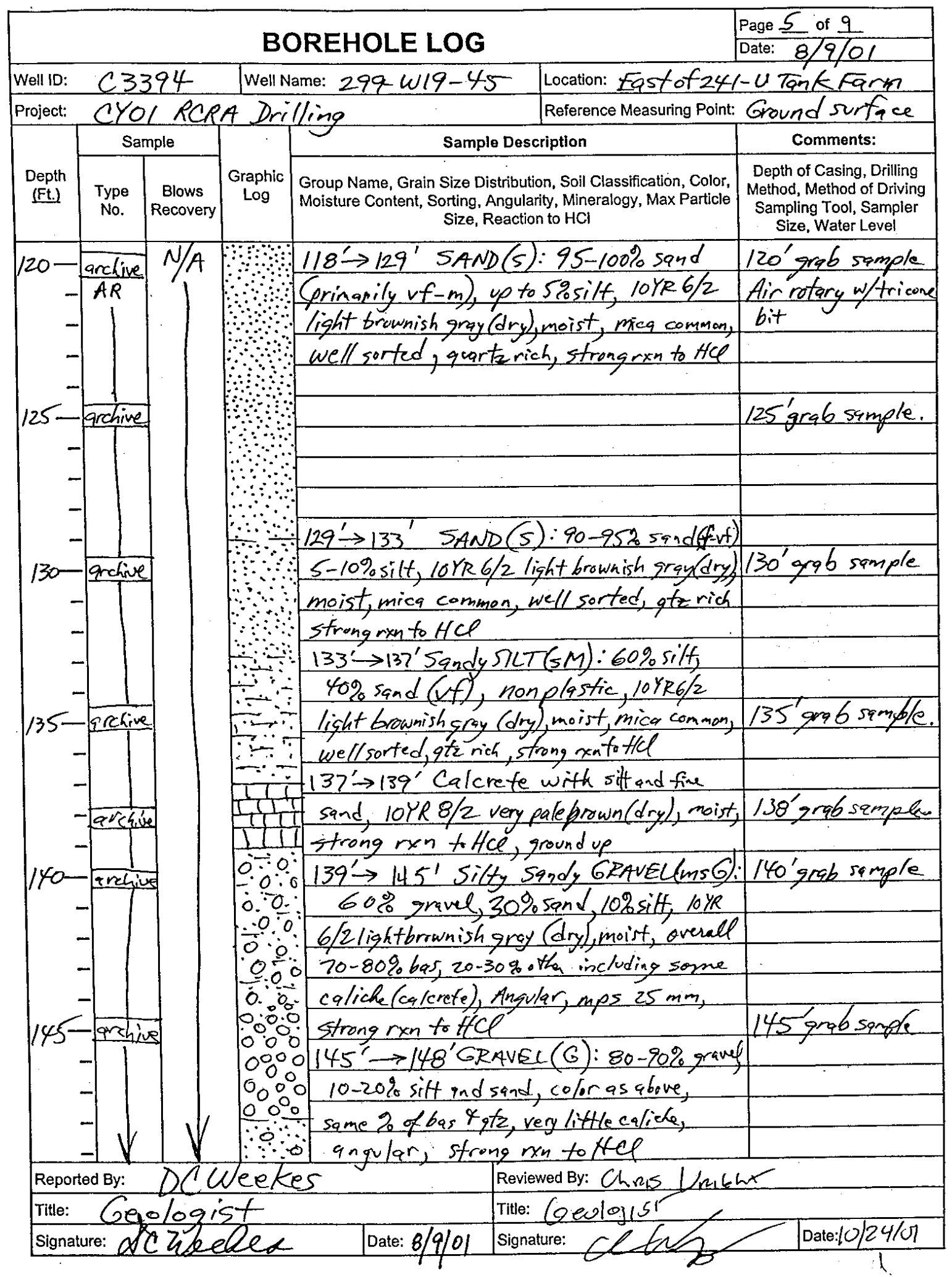

BHI-EE-183 (12/97) 


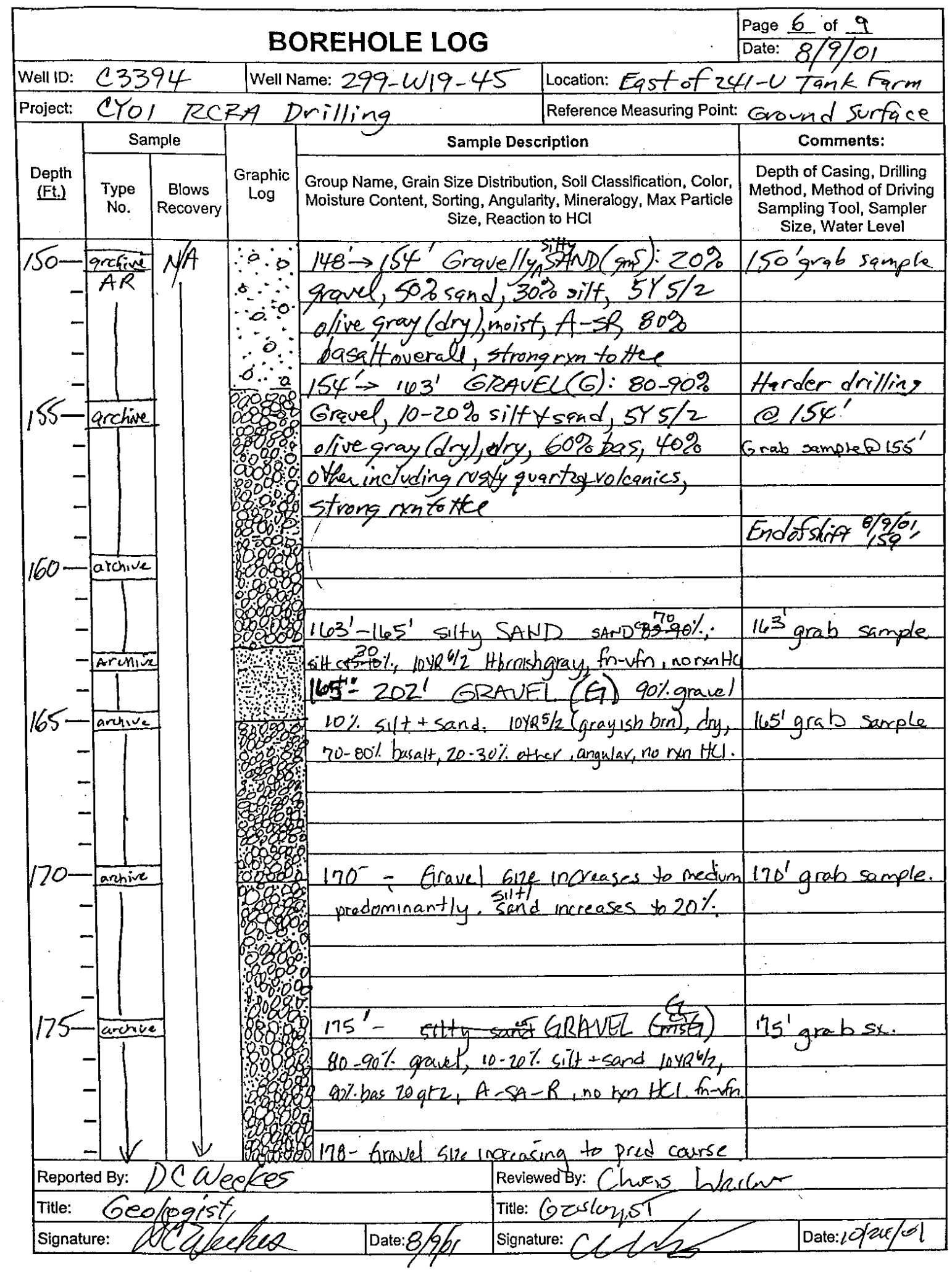

BHI-EE-183 (12/97) 


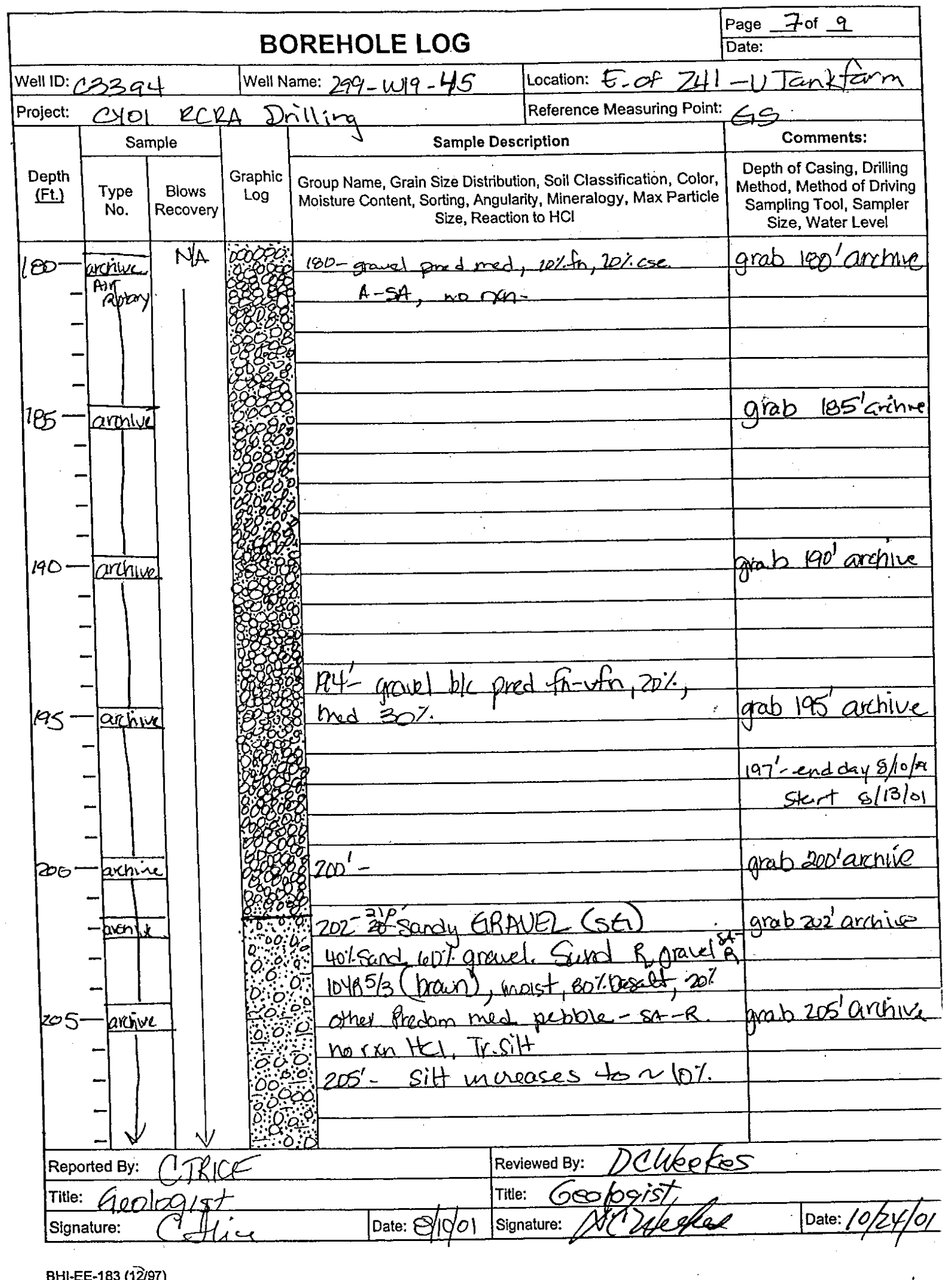




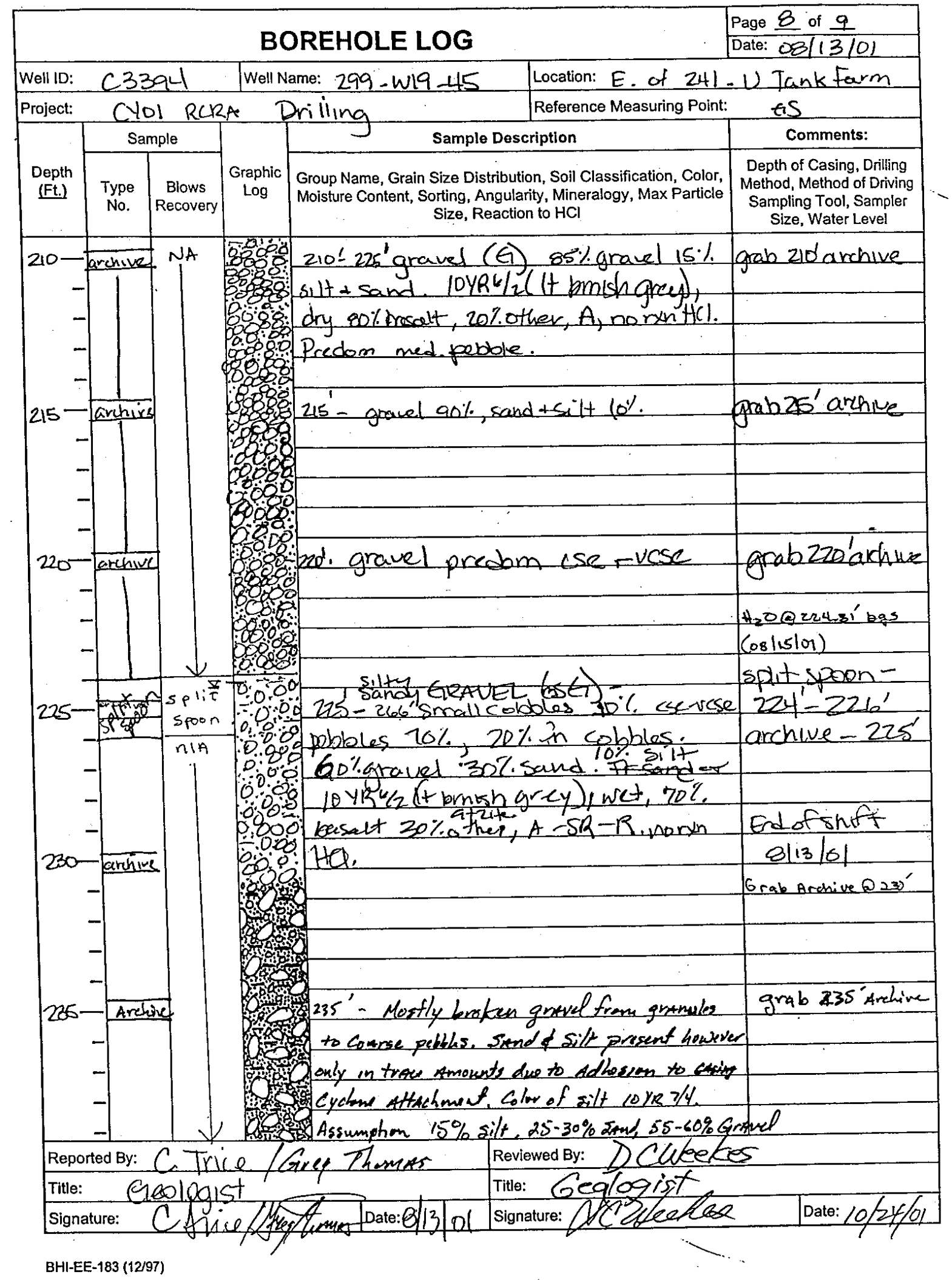




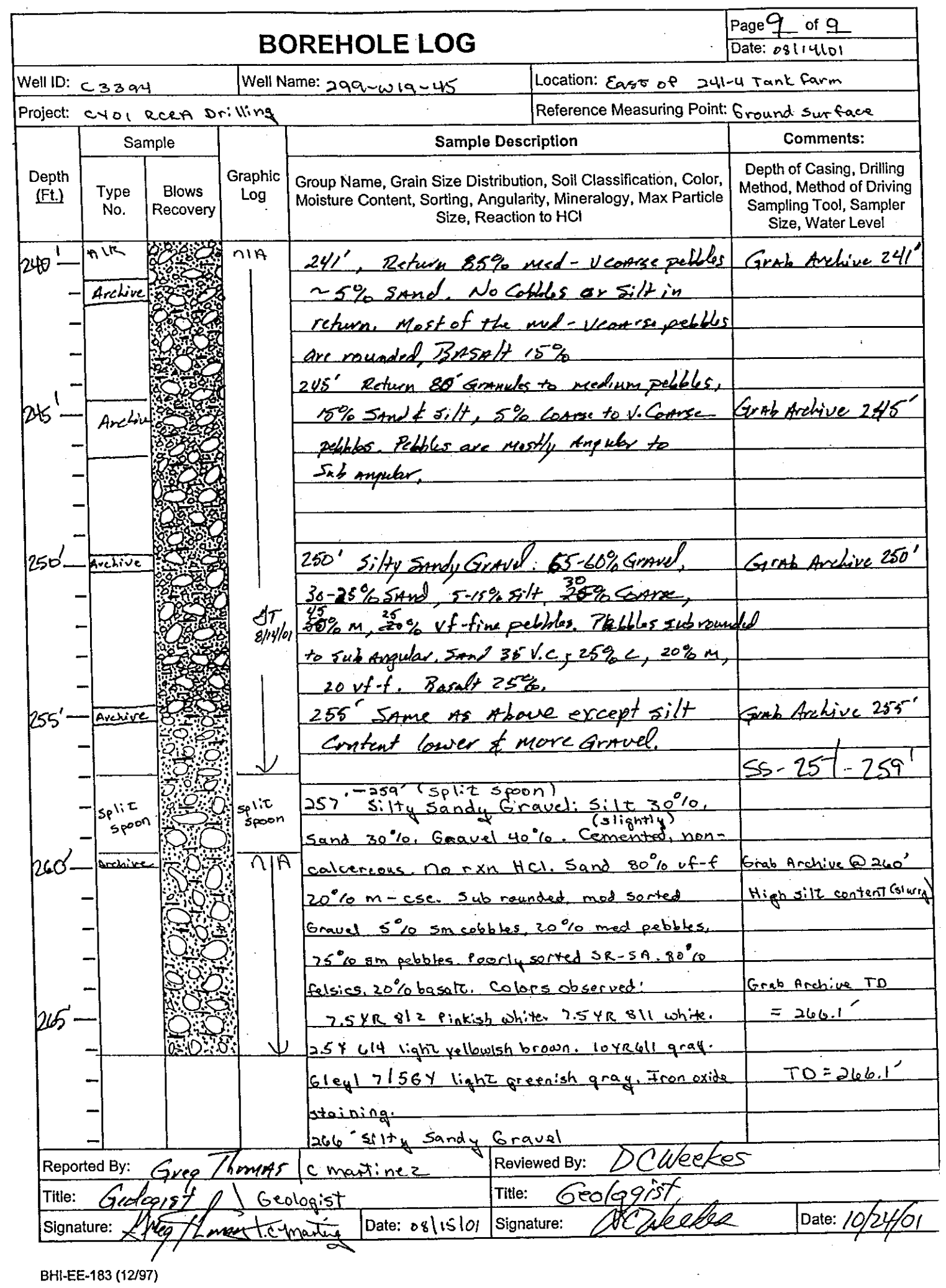




\section{Appendix B}

Physical Properties Data 


\section{Appendix B}

\section{Physical Properties Data}

This appendix includes the results of testing for particle size distribution on split spoon samples from the wells 299-W18-40, 299-W19-44, and 299-W19-45. The analyses were done by CH2M HILL Hanford, Inc. using standard sieve techniques. 


\begin{tabular}{|c|c|c|c|c|c|c|}
\hline \multicolumn{6}{|c|}{ CH2w Hill Hanford, Inc. } & \\
\hline WELL NAME & $299-W 18-40$ & DEPTH & $220.0^{\prime}-222.5^{\prime}$ & SAMPLE\# & W18-40-220.0 & WELL ID\# \\
\hline \multirow[t]{2}{*}{ TESTED BY } & J.MWimett & CONTACT & Dave Weekes & PHONE & $372-8430$ & DATE $\quad 10 / 16 / 2001$ \\
\hline & & & & \multicolumn{3}{|c|}{$9601 x^{20}$} \\
\hline $\begin{array}{l}\text { SAMPLE } \\
\text { WT }(g)\end{array}$ & $\begin{array}{c}\text { SIEVE } \\
\text { SIZE IN. }\end{array}$ & $\begin{array}{l}\text { CUMULATIVE } \\
\text { WEIGHT(g) }\end{array}$ & $\begin{array}{l}\% \text { WEIGHT } \\
\text { RETAINED }\end{array}$ & $\begin{array}{c}\% \\
\text { PASSING } \\
\end{array}$ & $\begin{array}{c}\text { Grain Size } \\
(\mathrm{mm})\end{array}$ & COMMENTS \\
\hline 980.90 & $2^{\prime \prime}$ & 0.0 & 0.0 & 100.0 & 50.80 & \\
\hline & $1.5^{\prime \prime}$ & 0.0 & 0.0 & 100.0 & 38.10 & \\
\hline & $3 / 4^{11}$ & 150.2 & 15.3 & 84.7 & 19.05 & \\
\hline & $3 / 8 "$ & 239.9 & 24.5 & 75.5 & 9.42 & \\
\hline & \#4 & 328.2 & 33.5 & 66.5 & 4.70 & \\
\hline & $\# 10$ & 469.7 & 47.9 & 52.1 & 1.98 & \\
\hline & $\# 20$ & 588.5 & 60.0 & 40.0 & 0.83 & \\
\hline & $\# 40$ & 677.5 & 69.1 & 30.9 & 0.42 & \\
\hline & $\# 60$ & 767.6 & 78.3 & 21.7 & 0.25 & \\
\hline & $\# 100$ & 822.4 & 83.8 & 16.2 & 0.150 & \\
\hline & $\# 200$ & 869.6 & 88.7 & 11.3 & 0.074 & \\
\hline
\end{tabular}

Sieve Analysis Data for Sample W18-40-220.0

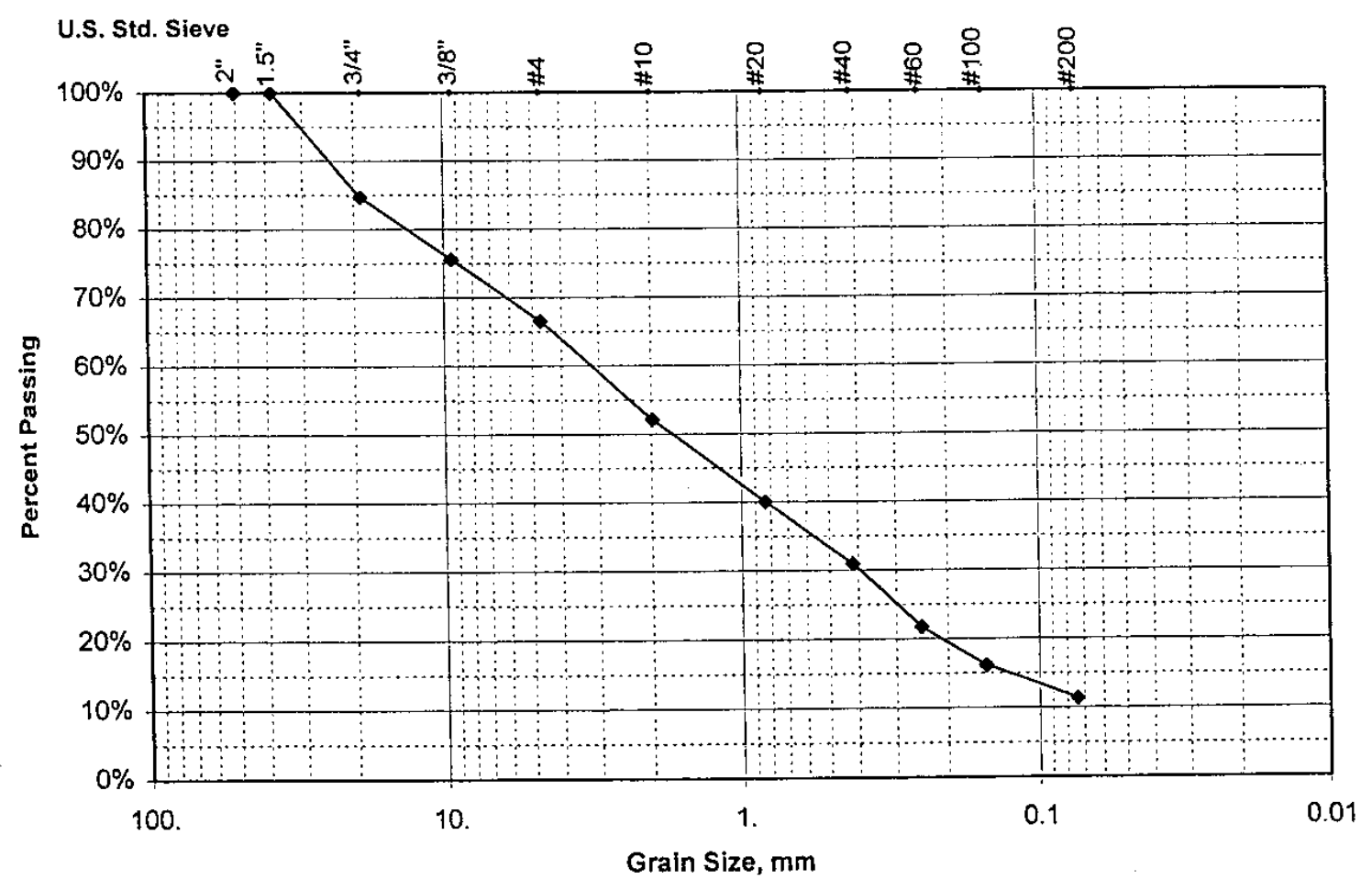

Comments: Silty Sandy Gravel

All data are accuratgly and completely recorded. Checked By: DC Zibeher /DCuleeker Date: $10 / 19 / 01$ 


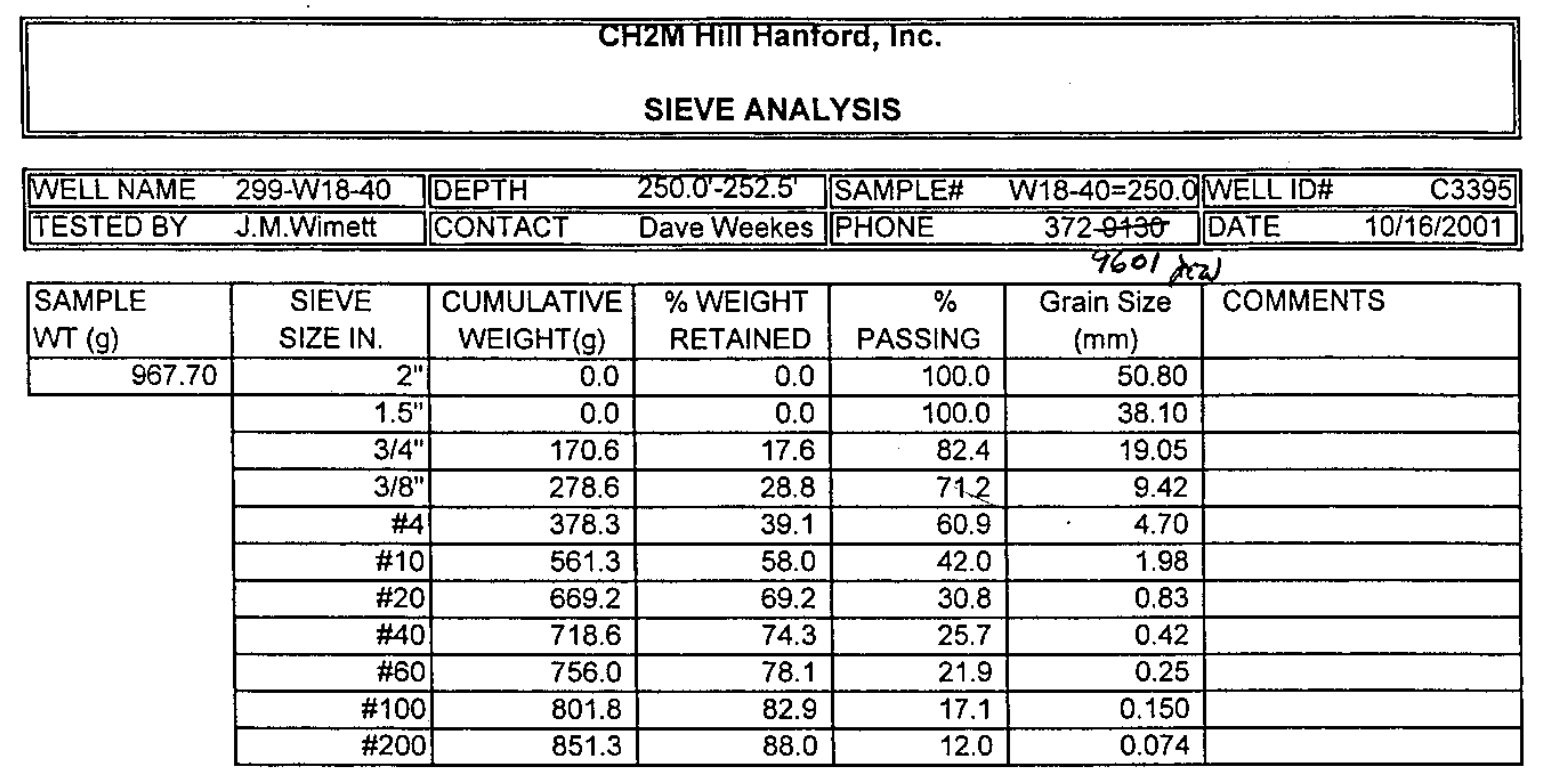

\section{Sieve Analysis Data for Sample W18 $-40=250.0$}

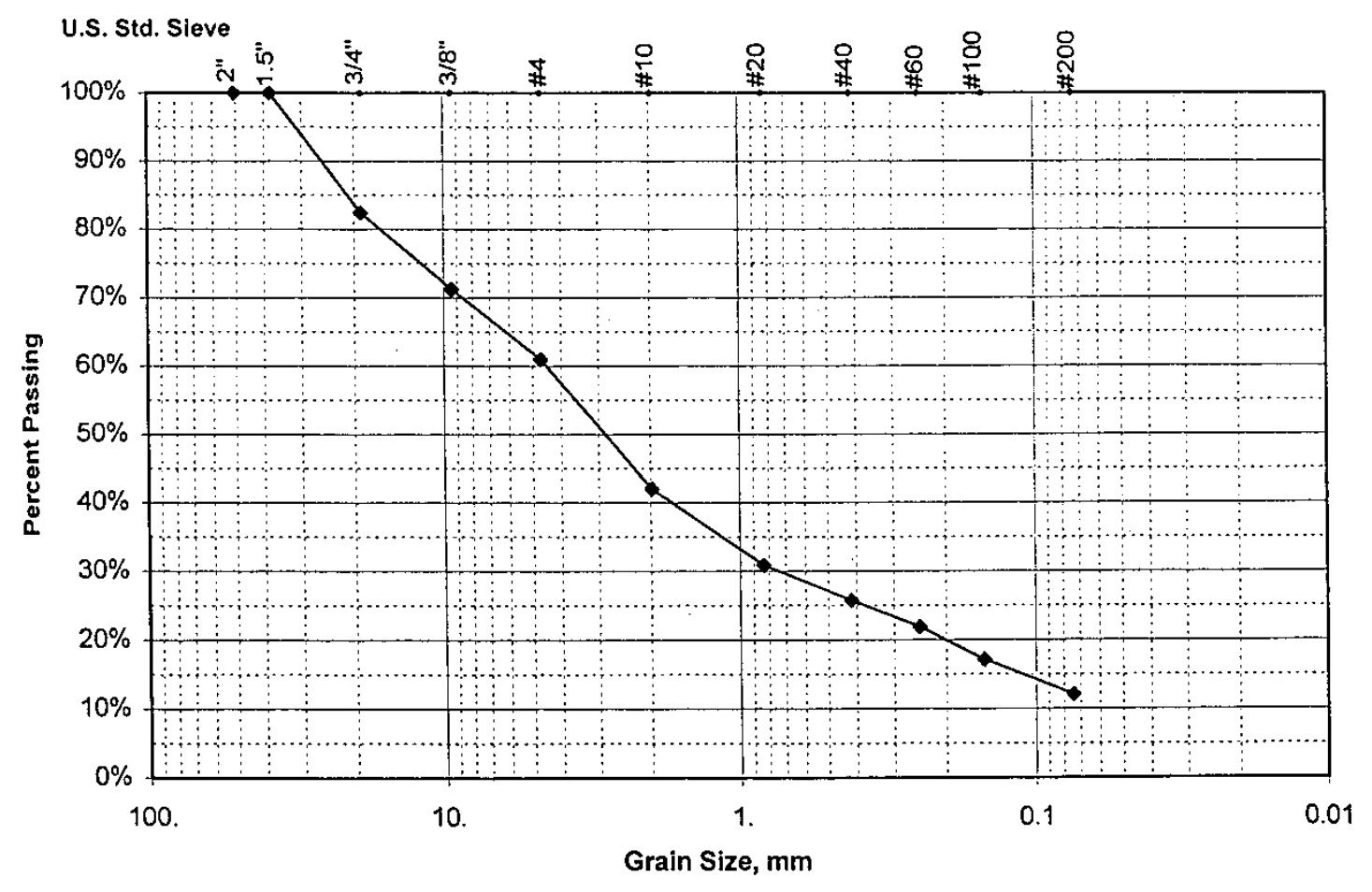

Comments: Silty Sandy Gravel

All data are accurately and completely recorded.

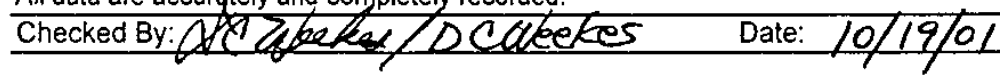




\begin{tabular}{|c|c|c|c|c|c|c|}
\hline & & & $\begin{array}{l}\text { T2M Hill Hanf } \\
\text { SIEVE ANAL }\end{array}$ & $\begin{array}{l}\text { ord, Inc. } \\
\text { YSIS }\end{array}$ & & \\
\hline WELL NAME & 299-W19-44 & $\overline{\text { DEPTH }}$ & $232.0^{\prime}-234.5^{\top}$ & SAMPLE\# & W19-44-232.0 & WELL ID\# \\
\hline TESTED BY & J.M.Wimett & CONTACT & Dave Weekes & PHONE & $372-0430$ & DATE $\quad 09 / 06 / 2001$ \\
\hline & & & & & 760 & $\frac{1}{W}$ \\
\hline $\begin{array}{l}\text { SAMPLE } \\
W T(g)\end{array}$ & $\begin{array}{l}\text { SIEVE } \\
\text { SIZE IN. }\end{array}$ & $\begin{array}{l}\text { CUMULATIVE } \\
\text { WEIGHT(g) }\end{array}$ & $\begin{array}{l}\text { \% WEIGHT } \\
\text { RETAINED }\end{array}$ & $\begin{array}{c}\% \\
\text { PASSING }\end{array}$ & $\begin{array}{c}\text { Grain Size } \\
(\mathrm{mm})\end{array}$ & COMMENTS \\
\hline 950.60 & $2^{\prime \prime}$ & 0.0 & 0.0 & 100.0 & 50.80 & \\
\hline & $1.5^{\prime \prime}$ & 0.0 & 0.0 & 100.0 & 38.10 & \\
\hline & $3 / 4^{11}$ & 135.0 & 14.2 & 85.8 & 19.05 & \\
\hline & $3 / 8^{\prime \prime}$ & 261.4 & 27.5 & 72.5 & 9.42 & \\
\hline & $\# 4$ & 329.4 & 34.7 & 65.3 & 4.70 & \\
\hline & $\# 10$ & 486.0 & $\overline{51.1}$ & 48.9 & 1.98 & \\
\hline & $\# 20$ & 622.6 & 65.5 & 34.5 & 0.83 & \\
\hline & $\# 40$ & 680.0 & 71.5 & 28.5 & 0.42 & \\
\hline & $\# 60$ & 729.9 & 76.8 & 23.2 & 0.25 & \\
\hline & $\# 100$ & 785.2 & 82.6 & 17.4 & 0.150 & \\
\hline & $\# 200$ & 834.6 & 87.8 & 12.2 & 0.074 & \\
\hline
\end{tabular}

Sieve Analysis Data for Sample W19-44-232.0

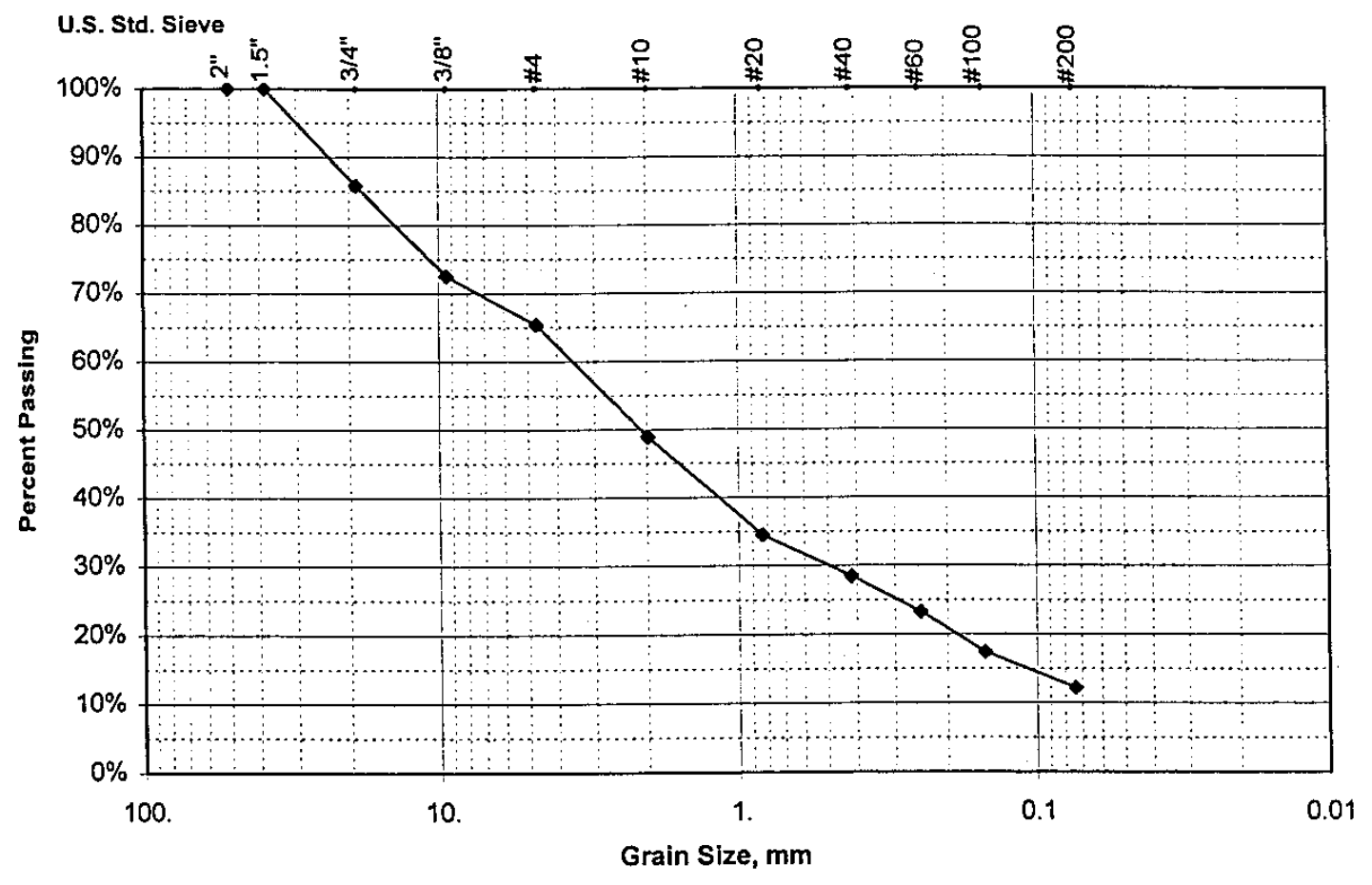

Comments: Silty Sandy Gravel

All data are accurfely and completely recorded.

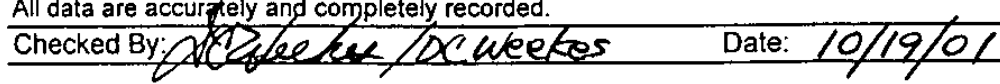




\begin{tabular}{|c|c|c|c|c|c|c|}
\hline \multicolumn{7}{|c|}{ CH2M Hili Hantord, Inc. } \\
\hline \multirow{2}{*}{$\begin{array}{l}\text { WELL NAME } \\
\text { TESTED BY } \\
\end{array}$} & 299-W19-44 & DEPTH & $267.0-269.5$ & SAMPLE\# & $\bar{W}+9-44-232.0$ & $\overline{\text { WELL ID\# }}$ \\
\hline & J.M.Wimett & CONTACT & Dave Weekes & PHONE & $372-0430$ & $09 / 06 / 2001$ \\
\hline & & & & \multicolumn{3}{|c|}{$9601 \mathrm{kol}$} \\
\hline $\begin{array}{l}\text { SAMPLE } \\
W T(g)\end{array}$ & $\begin{array}{c}\text { SIEVE } \\
\text { SIZE IN. }\end{array}$ & $\begin{array}{l}\text { CUMULATIVE } \\
\text { WEIGHT(g) }\end{array}$ & $\begin{array}{l}\text { \% WEIGHT } \\
\text { RETAINED }\end{array}$ & $\begin{array}{c}\% \\
\text { PASSING }\end{array}$ & $\begin{array}{c}\text { Grain Size } \\
(\mathrm{mm})\end{array}$ & COMMENTS \\
\hline 981.70 & $2^{\prime \prime}$ & 0.0 & 0.0 & 100.0 & 50.80 & \\
\hline & $1.5^{\prime \prime}$ & 0.0 & 0.0 & 100.0 & 38.10 & \\
\hline & $3 / 4^{\prime \prime}$ & 110.2 & 11.2 & 88.8 & 19.05 & \\
\hline & $3 / 8^{114}$ & 252.7 & 25.7 & 74.3 & 9.42 & \\
\hline & $\# 4$ & 328.7 & 33.5 & 66.5 & 4.70 & \\
\hline & $\# 10$ & 441.3 & 45.0 & 55.0 & 1.98 & \\
\hline & $\# 20$ & 550.1 & 56.0 & 44.0 & 0.83 & \\
\hline & $\# 40$ & 634.8 & 64.7 & 35.3 & 0.42 & \\
\hline & $\# 60$ & 731.5 & 74.5 & 25.5 & 0.25 & \\
\hline & $\# 100$ & 820.5 & 83.6 & 16.4 & 0.150 & \\
\hline & $\# 200$ & 870.9 & 88.7 & 11.3 & 0.074 & \\
\hline
\end{tabular}

\section{Sieve Analysis Data for Sample W19-44-232.0}

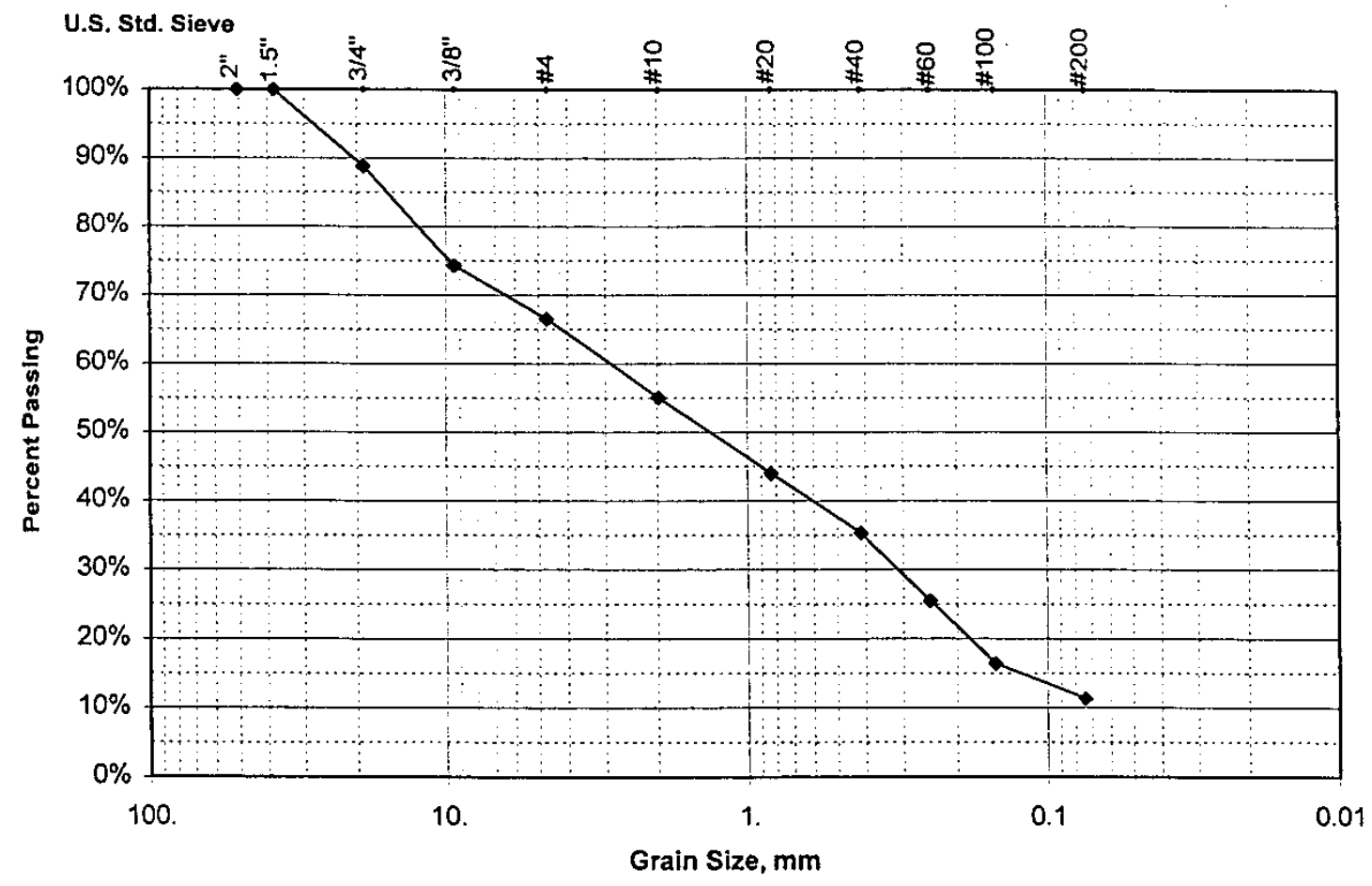

Comments: Silty Sandy Gravel

All data are accurately and completely recorded. 


\begin{tabular}{|c|c|c|c|c|c|c|}
\hline \multicolumn{7}{|c|}{$\begin{array}{c}\text { CH2M Hill Hanford, Inc. } \\
\text { SIEVE ANALYSIS }\end{array}$} \\
\hline \multirow{3}{*}{$\begin{array}{l}\text { WELL NAME } \\
\text { TESTED BY }\end{array}$} & $299-W 19-45$ & DEPTH & $224.0^{\prime}-226.0^{\prime}$ & SAMPLE\# & W19-45-224.0 & WELL ID\# \\
\hline & J.M.Wimett & $\overline{\mathrm{CONTACT}}$ & Dave Weekes & PHONE & $372-9430$ & $09 / 13 / 2001$ \\
\hline & & & & \multicolumn{3}{|c|}{$4601 \mathrm{~kg}$} \\
\hline $\begin{array}{l}\text { SAMPLE } \\
\text { WT (g) }\end{array}$ & $\begin{array}{c}\text { SIEVE } \\
\text { SIZE IN. }\end{array}$ & $\begin{array}{l}\text { CUMULATIVE } \\
\text { WEIGHT(g) }\end{array}$ & $\begin{array}{l}\text { \% WEIGHT } \\
\text { RETAINED }\end{array}$ & $\begin{array}{c}\% \\
\text { PASSING }\end{array}$ & $\begin{array}{c}\text { Grain Size } \\
\text { (mm) }\end{array}$ & COMMENTS \\
\hline \multirow[t]{11}{*}{980.00} & $2^{\prime \prime}$ & 0.0 & 0.0 & 100.0 & 50.80 & \\
\hline & $1.5^{\prime \prime}$ & 0.0 & 0.0 & 100.0 & 38.10 & \\
\hline & $3 / 4^{\prime \prime}$ & 397.3 & $\overline{40.5}$ & 59.5 & 19.05 & \\
\hline & $3 / 8^{\prime \prime}$ & 674.3 & 68.8 & 31.2 & 9.42 & \\
\hline & $\# 4$ & 766.8 & 78.2 & 21.8 & 4.70 & \\
\hline & $\# 10$ & 816.0 & 83.3 & 16.7 & 1.98 & \\
\hline & $\# 20$ & 842.3 & 85.9 & 14.1 & 0.83 & \\
\hline & $\# 40$ & 858.9 & 87.6 & 12.4 & 0.42 & \\
\hline & $\# 60$ & 892.9 & 91.1 & 8.9 & 0.25 & \\
\hline & $\# 100$ & 927.6 & 94.7 & 5.3 & 0.150 & \\
\hline & $\# 200$ & 947.9 & 96.7 & 3.3 & 0.074 & \\
\hline
\end{tabular}

Sieve Analysis Data for Sample W19-45-224.0

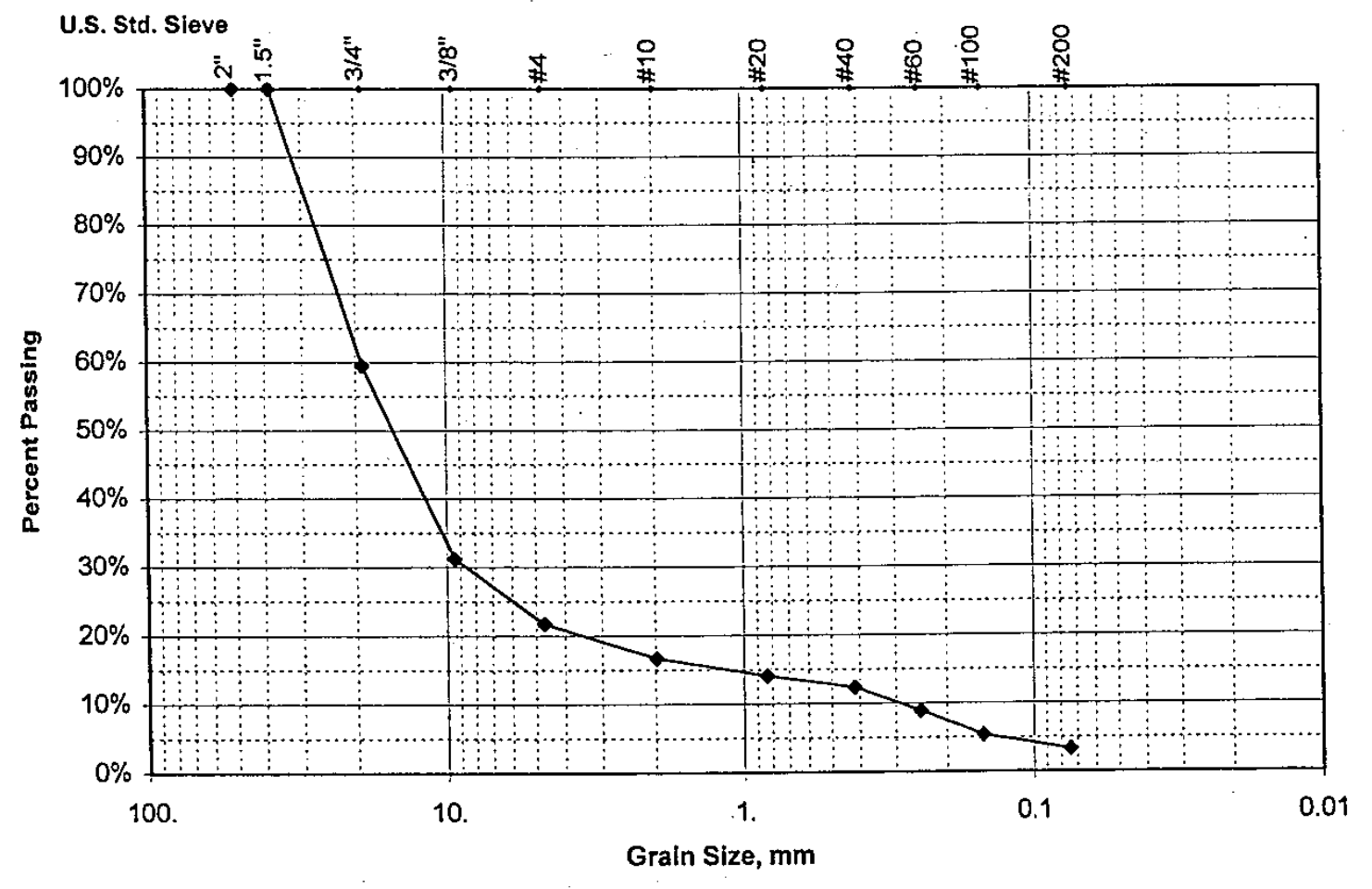

Comments: Sandy Gravel

All data are accurately and,completely, recorded.

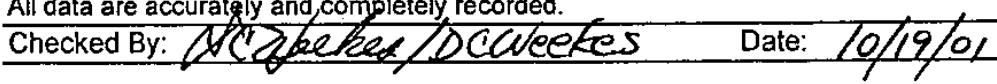

B.6 


\begin{tabular}{|c|c|c|c|c|c|c|}
\hline & & & \multicolumn{2}{|c|}{ SIEVE ANALYSIS } & & \\
\hline WELL NAME & $299-W 19-45$ & DEPTH & $258.0^{\circ}-259.0^{\circ}$ & SAMPLE\# & W19-45-258.0] & WELL ID\# \\
\hline TESTED BY & J.M.Wimett & CONTACT & Dave Weekes & PHONE & $372-9130$ & $09 / 13 / 2001$ \\
\hline $\begin{array}{l}\text { SAMPLE } \\
\text { WT }(g)\end{array}$ & $\begin{array}{c}\text { SIEVE } \\
\text { SIZE IN. }\end{array}$ & $\begin{array}{l}\text { CUMULATIVE } \\
\text { WEIGHT(g) }\end{array}$ & $\begin{array}{l}\text { \% WEIGHT } \\
\text { RETAINED }\end{array}$ & $\begin{array}{c}\% \\
\text { PASSING } \\
\end{array}$ & $\begin{array}{c}\text { Grain Size } \\
(\mathrm{mm})\end{array}$ & COMMENTS \\
\hline 976.90 & 21 & 0.0 & 0.0 & 100.0 & 50.80 & \\
\hline & $1.5^{\prime \prime}$ & 93.7 & 9.6 & 90.4 & 38.10 & \\
\hline & $3 / 4^{\prime \prime}$ & 272.3 & 27.9 & 72.1 & 19.05 & \\
\hline & $3 / 8^{\prime \prime}$ & 453.9 & 46.5 & 53.5 & 9.42 & \\
\hline & $\# 4$ & 550.8 & 56.4 & 43.6 & 4.70 & \\
\hline & $\# 10$ & 644.2 & 65.9 & 34.1 & 1.98 & \\
\hline & $\# 20$ & 693.5 & 71.0 & 29.0 & 0.83 & \\
\hline & $\# 40$ & 723.3 & 74.0 & 26.0 & 0.42 & \\
\hline & $\# 60$ & 777.3 & 79.6 & 20.4 & 0.25 & \\
\hline & $\# 100$ & 835.2 & 85.5 & 14.5 & 0.150 & \\
\hline & $\# 200$ & 875.0 & 89.6 & 10.4 & 0.074 & \\
\hline
\end{tabular}

Sieve Analysis Data for Sample W19-45-258.0

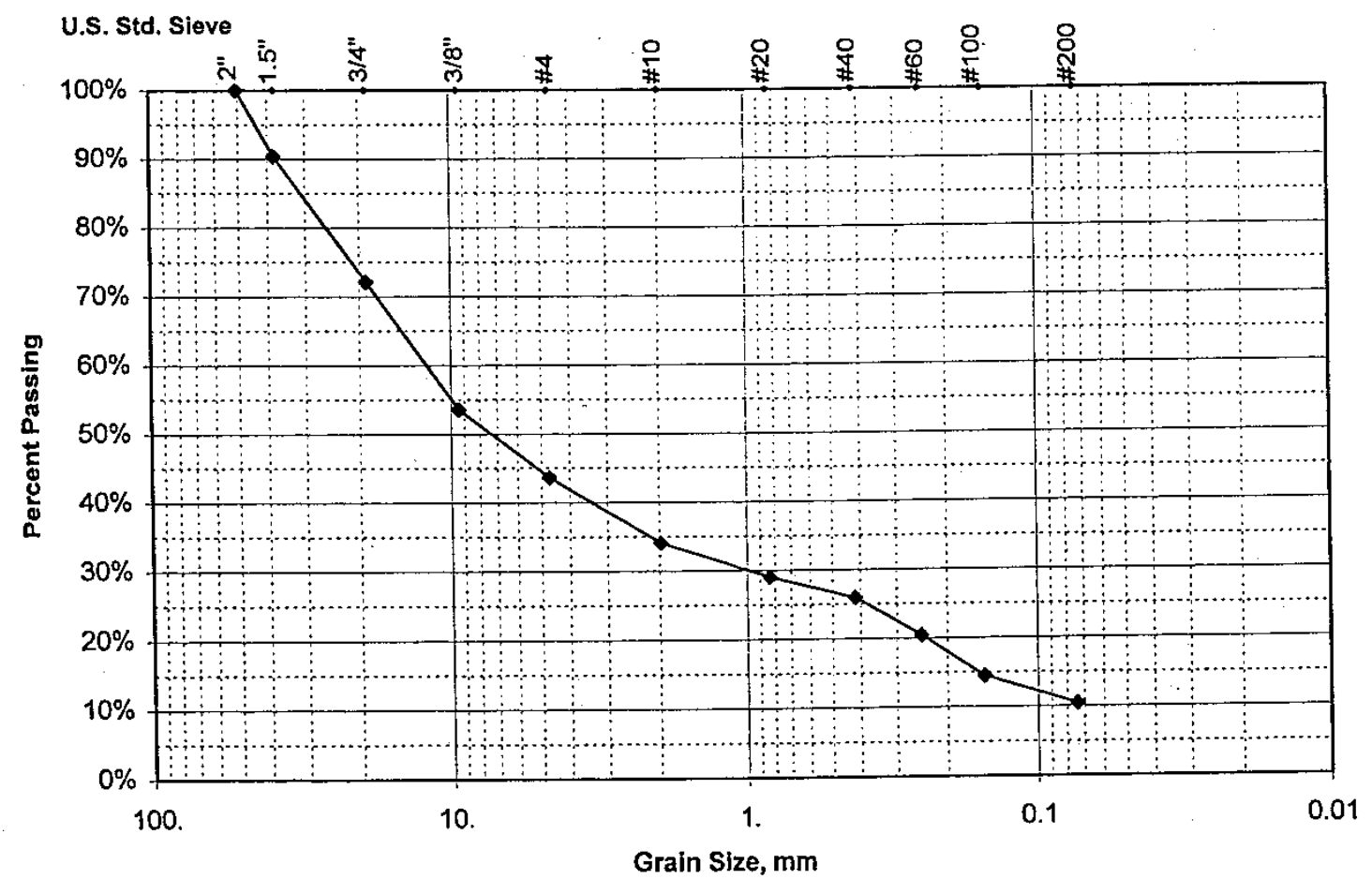

Comments: Silty Sandy Gravel

All data are accurately and completely recorded.

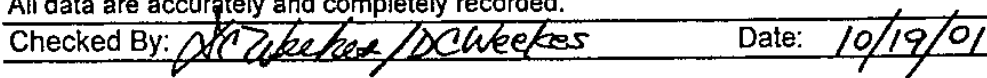

B. 7 


\section{Appendix C}

\section{Borehole Geophysical Logs}




\section{Appendix C}

\section{Borehole Geophysical Logs}

This appendix contains the borehole geophysical logs obtained from boreholes 299-W18-40, 299-W19-44, and 299-W19-45. The logs were run and analyzed by MACTEC-ERS. The Log Data Reports are included with the logs.

C. 1 


\section{9-W18-40(C3395)}

\section{Log Data Report}

\section{Borehole Information:}

\begin{tabular}{|cc|c|c|c|c|}
\hline Borehole: & 299-W18-40 (C3395) & \multicolumn{3}{c|}{ Site: } & \multicolumn{2}{c|}{ South of U Tank Farm } \\
\hline Coordinates (Plant) & GWL (ft) $:$ & 228 & GWL Date: & $9 / 19 / 01$ \\
\cline { 2 - 6 } North & East & Drill Date & ToC'Elevation & Total Depth (ft) & Type \\
Unknown & Unknown & Sept. 2001 & Unknown & 260 & Cable Tool \\
\hline
\end{tabular}

\section{Casing Information:}

\begin{tabular}{|lcccccc|}
\hline Casing Type & Stickup (ft) & $\begin{array}{c}\text { Outer } \\
\text { Diameter } \\
\text { (in.) }\end{array}$ & $\begin{array}{c}\text { Inside } \\
\text { Diameter } \\
\text { (in.) }\end{array}$ & $\begin{array}{c}\text { Thickness } \\
\text { (in.) }\end{array}$ & $\begin{array}{c}\text { Top } \\
\text { (ft) }\end{array}$ & $\begin{array}{c}\text { Bottom } \\
\text { (ft) }\end{array}$ \\
\hline Steel & 2.7 & $107 / 8$ & $93 / 8$ & $11 / 16$ & 2.7 & 258 \\
\hline
\end{tabular}

\section{Borehole Notes:}

The BHI site geologist reported the GWL as an approximate depth. The logging engineer measured the pipe stickup at the borehole using a steel tape. Calipers were used to measure casing OD and thickness only; the casing ID is calculated.

\section{Logging Equipment Information:}

\begin{tabular}{|c|c|c|c|}
\hline Logging System: & Gamma 2A & & Type: SGLS (35\%) \\
\hline Calibration Date: & $09 / 00$ & Calibration Reference: & GJO-2001-246-JAR \\
\hline & & Logging Procedure: & MAC-HGLP 1.6.5 \\
\hline Logging System: & Gamma 2E & & Type: NMLS \\
\hline Calibration Date: & $05 / 01$ & Calibration Reference: & GJO-2001-247-TAR \\
\hline & & Logging Procedure: & MAC-HGLP 1.6.5 \\
\hline
\end{tabular}

\section{Spectral Gamma Logging System (SGLS) Log Run Information:}

\begin{tabular}{|l|c|c|c|c|}
\hline Log Run & $\mathbf{1}$ & $\mathbf{2}$ & $\mathbf{3}$ & $\mathbf{4}$ \\
\hline Date & $09 / 20 / 01$ & $09 / 20 / 01$ & $09 / 21 / 01$ & \\
\hline Logging Engineer & Spatz & Spatz & Spatz & \\
\hline Start Depth (ft) & 0 & 120.0 & 244.0 & \\
\hline Finish Depth (ft) & 121.0 & 260.0 & 218.0 & \\
\hline Count Time (sec) & 200 & 200 & 200 & \\
\hline Live/Real & $\mathrm{R}$ & $\mathrm{R}$ & $\mathrm{R}$ & \\
\hline Shield (Y/N) & $\mathrm{N} / \mathrm{A}$ & $\mathrm{N} / \mathrm{A}$ & $\mathrm{N} / \mathrm{A}$ & \\
\hline MSA Interval (ft) & 1.0 & 1.0 & 1.0 & \\
\hline ft/min & $\mathrm{N} / \mathrm{A}$ & $\mathrm{N} / \mathrm{A}$ & $\mathrm{N} / \mathrm{A}$ & \\
\hline Pre-Verification & $\mathrm{B} 0056 \mathrm{CAB}$ & $\mathrm{B} 0056 \mathrm{CAB}$ & $\mathrm{B} 0056 \mathrm{CAB}$ & \\
\hline Start File & $\mathrm{B} 0056000$ & $\mathrm{~B} 0056122$ & $\mathrm{~B} 0056263$ & \\
\hline Finish File & $\mathrm{B} 0056121$ & $\mathrm{~B} 0056262$ & $\mathrm{~B} 0056289$ & \\
\hline Post-Verification & $\mathrm{B} 0056 \mathrm{CAA}$ & $\mathrm{B} 0056 \mathrm{CAA}$ & $\mathrm{B} 0056 \mathrm{CAA}$ & \\
\hline Depth Return Error (ft) & +0.4 & $\mathrm{~N} / \mathrm{A}$ & -0.25 & \\
\hline Comments & & & & \\
\hline
\end{tabular}


Neutron Moisture Logging System (NMLS) Log Run Information:

\begin{tabular}{|c|c|c|c|c|}
\hline Log Run & 1 & 2 & 3 & 4 \\
\hline Date & $09 / 19 / 01$ & $09 / 20 / 01$ & & \\
\hline Logging Engineer & Spatz & Spatz & & \\
\hline Start Depth $(\mathrm{ft})$ & 0 & 198.0 & & \\
\hline Finish Depth $(\mathrm{ft})$ & 231.0 & 175.0 & & \\
\hline Count Time (sec) & 15 & 15 & & \\
\hline Live/Real & $\mathrm{L}$ & $\mathrm{L}$ & & \\
\hline Shield (Y/N) & N/A & $\mathrm{N} / \mathrm{A}$ & & \\
\hline MSA Interval (ft) & 0.25 & 0.25 & & \\
\hline $\mathrm{ft} / \mathrm{min}$ & $\mathbb{N} / \mathrm{A}$ & $\mathrm{N} / \mathrm{A}$ & & \\
\hline Pre-Verification & C0017CAB & $\mathrm{C} 0017 \mathrm{CAB}$ & & \\
\hline Start File & 00017000 & CoR17000 & & \\
\hline Finish File & C0017928 & CoR17092 & & \\
\hline Post-Verification & COR17CAA & COR17CAA & & \\
\hline Depth Return Error (ft) & $\mathrm{N} / \mathrm{A}$ & 0 & & \\
\hline Comments & $\begin{array}{l}\text { Water detected } \\
\text { below } 231.0 \mathrm{ft} \text {. }\end{array}$ & Repeat interval. & & \\
\hline
\end{tabular}

\section{Logging Operation Notes:}

Zero reference is the top of ground surface, and SGLS log depths are relative to ground level.

A longer count time ( $200 \mathrm{sec}$ ) was required with the SGLS because of the relatively thick casing. The borehole was logged in the drill pipe before completion as a groundwater monitoring well. In order to obtain reliable spectra while minimizing overall logging time, the depth interval was increased from $0.5 \mathrm{ft}$ to $1.0 \mathrm{ft}$.

Fine gain adjustments were made after files B0056013 (13.0 ft), B0056040 (40.0 ft), B0056059 (59.0 ft), and $\mathrm{B} 0056076$ (76.0 ft) during logging run 1.

Log run 1 was terminated to refill the sonde with liquid nitrogen and to grease the PTO driveline.

Two spectra, files B0056261 and B0056262, may be from the sonde sitting on the bottom of the borehole in thick watery mud and may not represent true depth intervals.

During logging, the sonde is centralized in the borehole for both the SGLS and NMLS,

\section{Analysis Notes:}

\section{\begin{tabular}{|l|l|l|l|l|l|} 
Analyst: & Sobczyk & Date: & $09 / 25 / 01$ & Reference: & MAC-VZCP 1.7.9 Rev. 2
\end{tabular}}

Pre-run and post-run verification spectra for the SGLS were evaluated. The pre-survey verification spectrum (file B00056CAB) was within the control limits. However, the peak intensity for the $609-\mathrm{keV}$ photopeak was below the lower warning limits for this pre-run verification spectrum. The post-survey verification spectrum for the logging (file B00056CAA) was below the lower control limits for all three of the peak intensities. On the basis of the acceptance criteria for the Gamma $2 \mathrm{~A}$ system, both the pre- and post-verification spectra did not fulfill the acceptance criteria. Examinations of spectra indicate that the detector appears to have functioned normally during the log run, and the log data are provisionally accepted, subject to further review and analysis. 
Individual spectra were processed in batch mode using APTEC SUPERVISOR to identify individual energy peaks and determine count rates. Concentrations were calculated with EXCEL. Corrections were applied for a casing thickness of $11 / 16 \mathrm{in}$. from the ground surface to $260 \mathrm{ft}$. A correction for water in the borehole was applied below $230 \mathrm{ft}$, and this depth was determined from the neutron-mois ture log. Dead time corrections were not necessary. The rerun of the SGLS showed good repeatability.

Pre-run and post-run verification spectra for the NMLS were evaluated. The pre-survey verification spectrum (file C0017CAB) recorded 723 gross cps while the post-survey verification spectrum (file CoR17CAA) recorded 747 gross cps.

Moisture calibration models at Hanford for 10-in. holes with 11/16-in. casing have not been established. Thus, the neutron log was not processed to estimate volumetric moisture content because the relatively large borehole diameter and casing thickness are beyond the range of conditions for which the tool was calibrated. Neutron data are presented as gross counts. In general, an increase in neutron count is indicative of an increase in moisture content, but a quantitative calculation of volumetric moisture cannot be made at this time. The rerun of the neutron-moisture tool showed good repeatability.

\section{Log Plot Notes:}

Separate $\log$ plots are provided for gross gamma, naturally occurring radionuclides $\left({ }^{40} \mathrm{~K},{ }^{232} \mathrm{Th},{ }^{238} \mathrm{U}\right.$, and associated decay progeny), and man-made radionuclides. For each radionuclide, the energy value of the spectral peak used for quantification is indicated. Unless otherwise noted, all radionuclides are plotted in picocuries per gram $(\mathrm{pCi} / \mathrm{g})$. The open circles indicate the minimum detectable level (MDL) for each radionuclide. Error bars on each plot represent error associated with counting statistics only and do not include errors associated with the inverse efficiency function, dead time correction, or casing and water corrections. These errors are discussed in the calibration report. A combination plot is also included to facilitate correlation. A gross neutron log of neutron counts is also shown on the combination plot.

\section{Results and Interpretations:}

${ }^{137} \mathrm{Cs}$ was the only man-made radionuclide detected. ${ }^{137} \mathrm{Cs}$ activity was detected at two points near the ground surface. The measured ${ }^{137} \mathrm{Cs}$ activity was about $0.2 \mathrm{pCi} / \mathrm{g}$ at both the ground surface and at a log depth of $3 \mathrm{ft}$.

The changes in gross gamma counts depend primarily upon changes in ${ }^{40} \mathrm{~K}$ activities. The increase in gross gamma counts from about $75 \mathrm{cps}$ to about $115 \mathrm{cps}$ at a $\log$ depth of $69 \mathrm{ft}$ corresponds with an increase in apparent ${ }^{40} \mathrm{~K}$ activity from about 10 to $15 \mathrm{pCi} / \mathrm{g}$. This increase in total gamma is interpreted as the Hanford $\mathrm{H} 2$. The increase in ${ }^{232} \mathrm{Th}$ activity from about 0.8 to $1.0 \mathrm{pCi} / \mathrm{g}$ and the increase in gross gamma counts from 110 to $125 \mathrm{cps}$ at $116 \mathrm{ft}$ probably represent the top of the Early Palouse Soil. On the basis of low K-40 activities, the carbonate-rich paleosols of the Pliocene-Pleistocene are interpreted as being between 133 and $137 \mathrm{ft}$. The caliche layer with characteristically high uranium content (greater than $2.0 \mathrm{pCi} / \mathrm{g}$ ) is present between 133 and $135 \mathrm{ft}$. The top of the Ringold is picked at $138 \mathrm{ft}$.

The neutron moisture tool's depressed response in this hole is due at least in part to the low-activity source, short source-to-detector spacing, and large borehole diameter. The highest neutron counts occurred in the groundwater as expected. The higher neutron counts that occurred in the 115- to $137-\mathrm{ft}$ interval correspond with the Plio-Pleistocene as interpreted from the SGLS data.

\footnotetext{
${ }^{1}$ GWL - groundwater level

${ }^{2}$ TOC - top of casing

${ }^{3} \mathrm{~N} / \mathrm{A}$ - not applicable
} 


\section{9-W18-40 (C3395) Man-Made Radionuclide Concentrations}

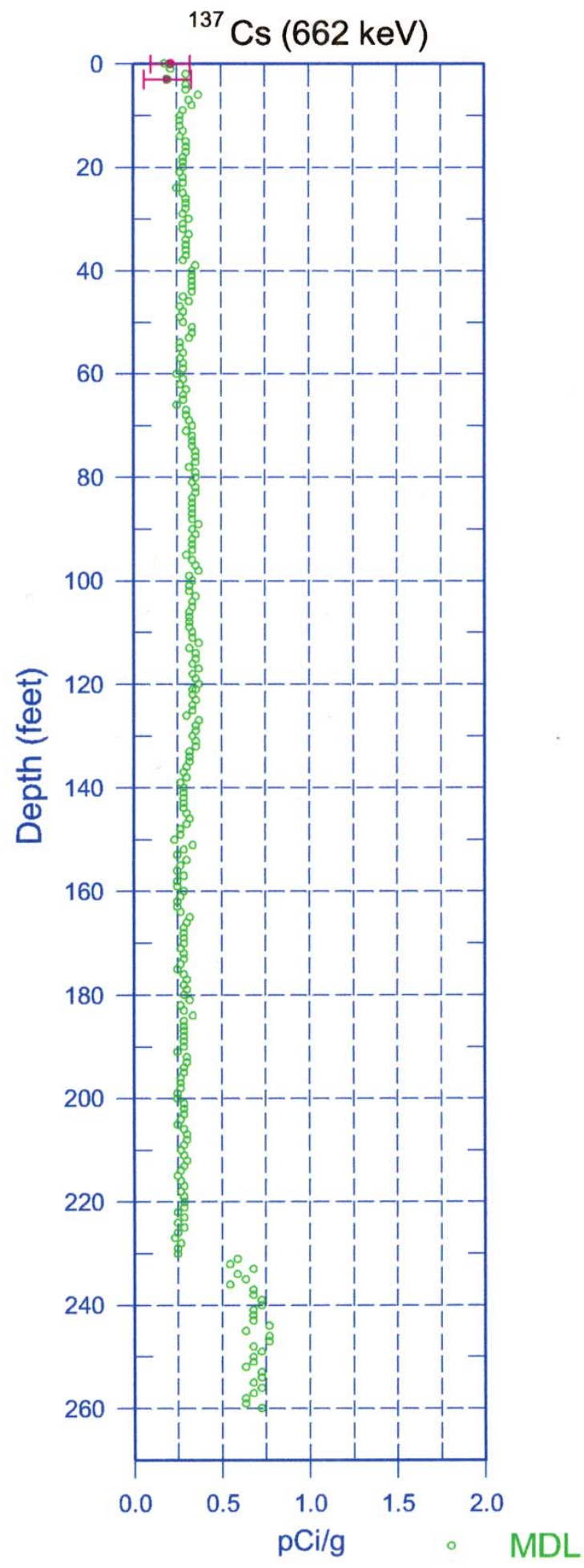

C.5 


\section{9-W18-40 (C3395) Natural Gamma Logs}
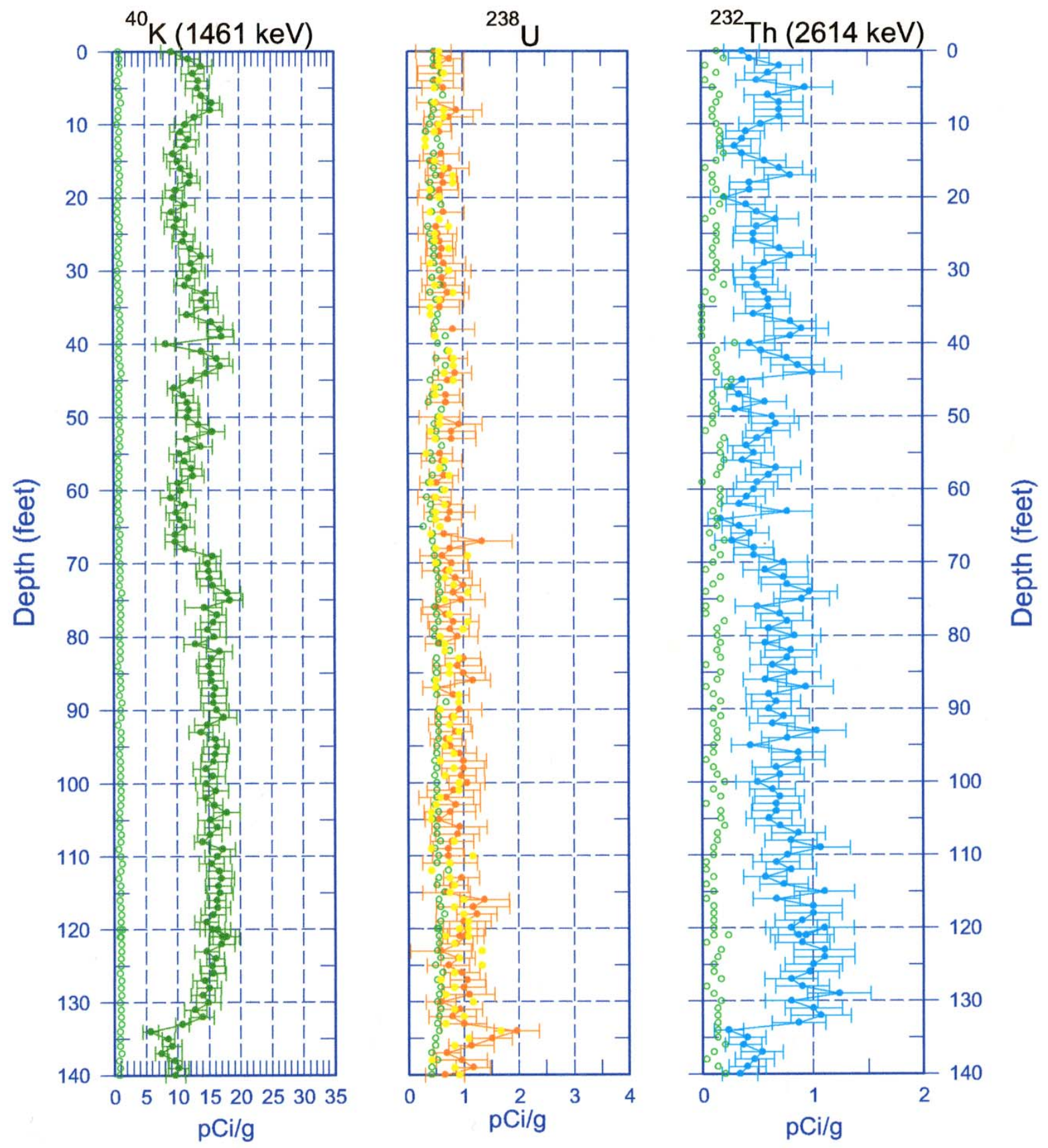

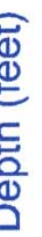

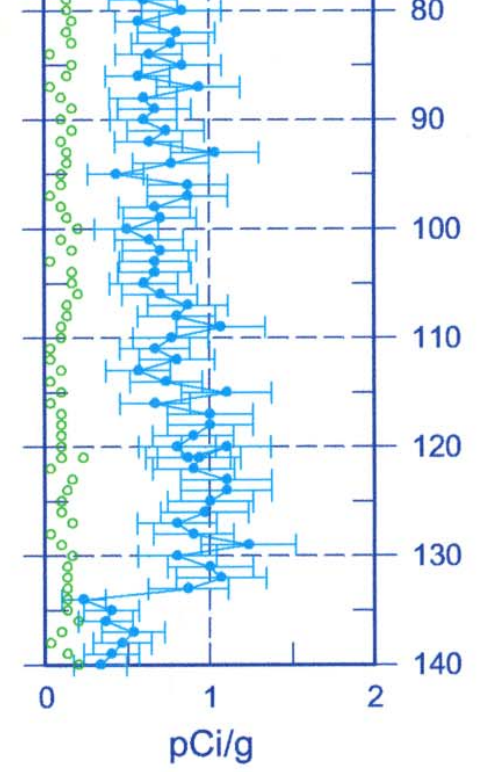

$$
\begin{array}{ll}
\because & 609 \mathrm{keV} \\
-\quad \mathrm{MDL} \\
-1764 \mathrm{keV}
\end{array}
$$

MDL

C. 6 


\section{9-W18-40 (C3395) Natural Gamma Logs}
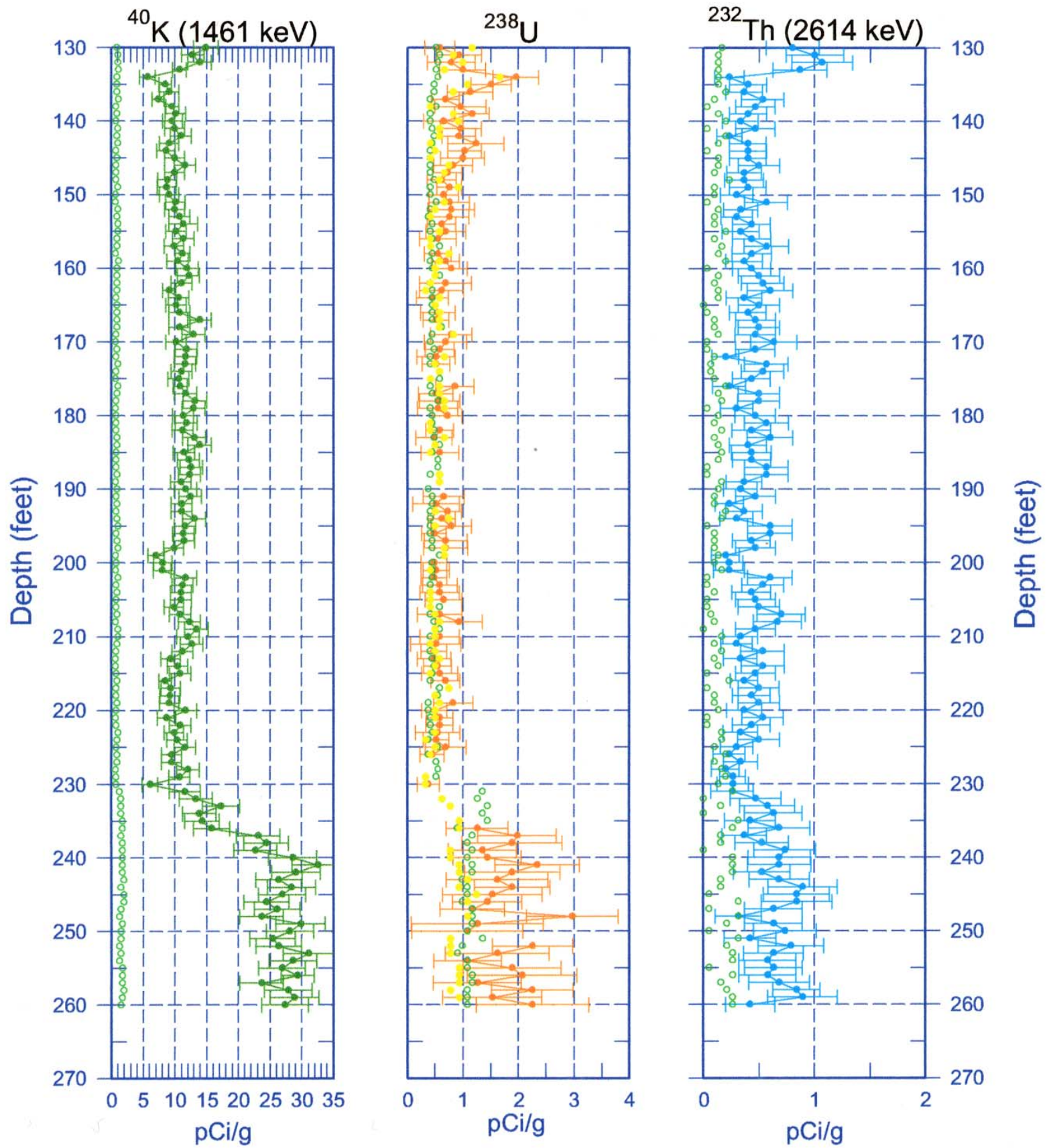

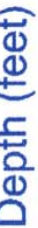
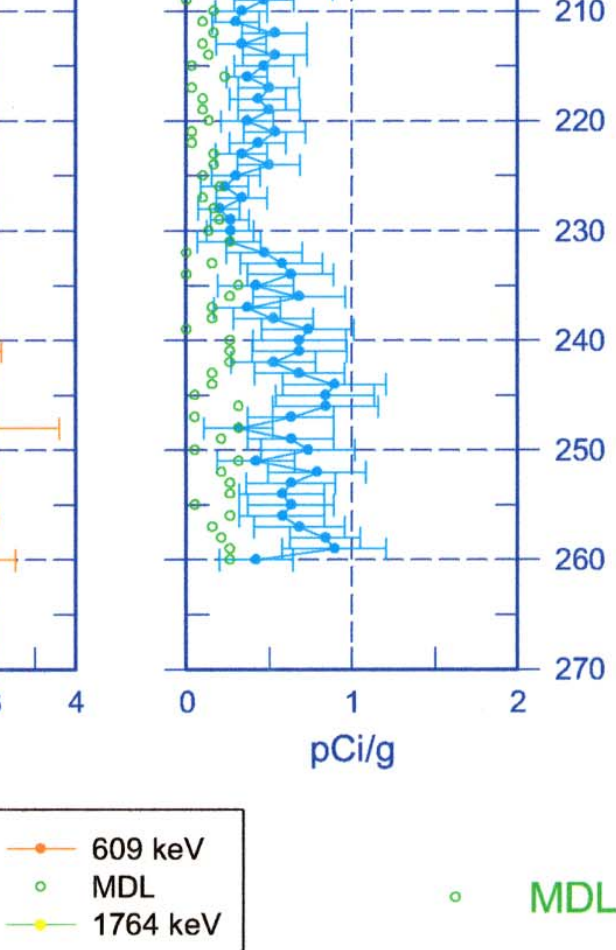

MDL

C.7 


\section{9-W40-18 (C3395) Combination Plot}

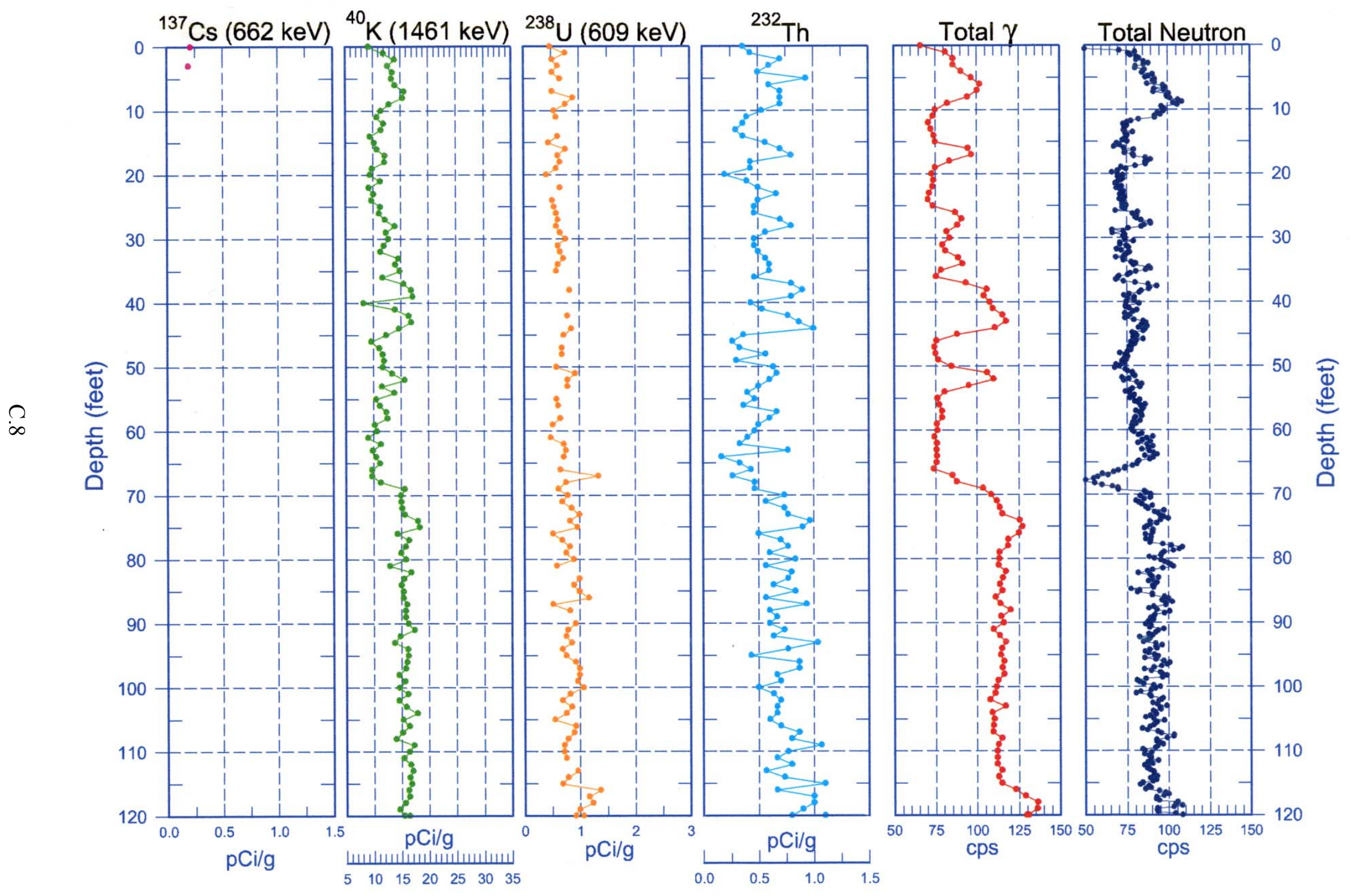




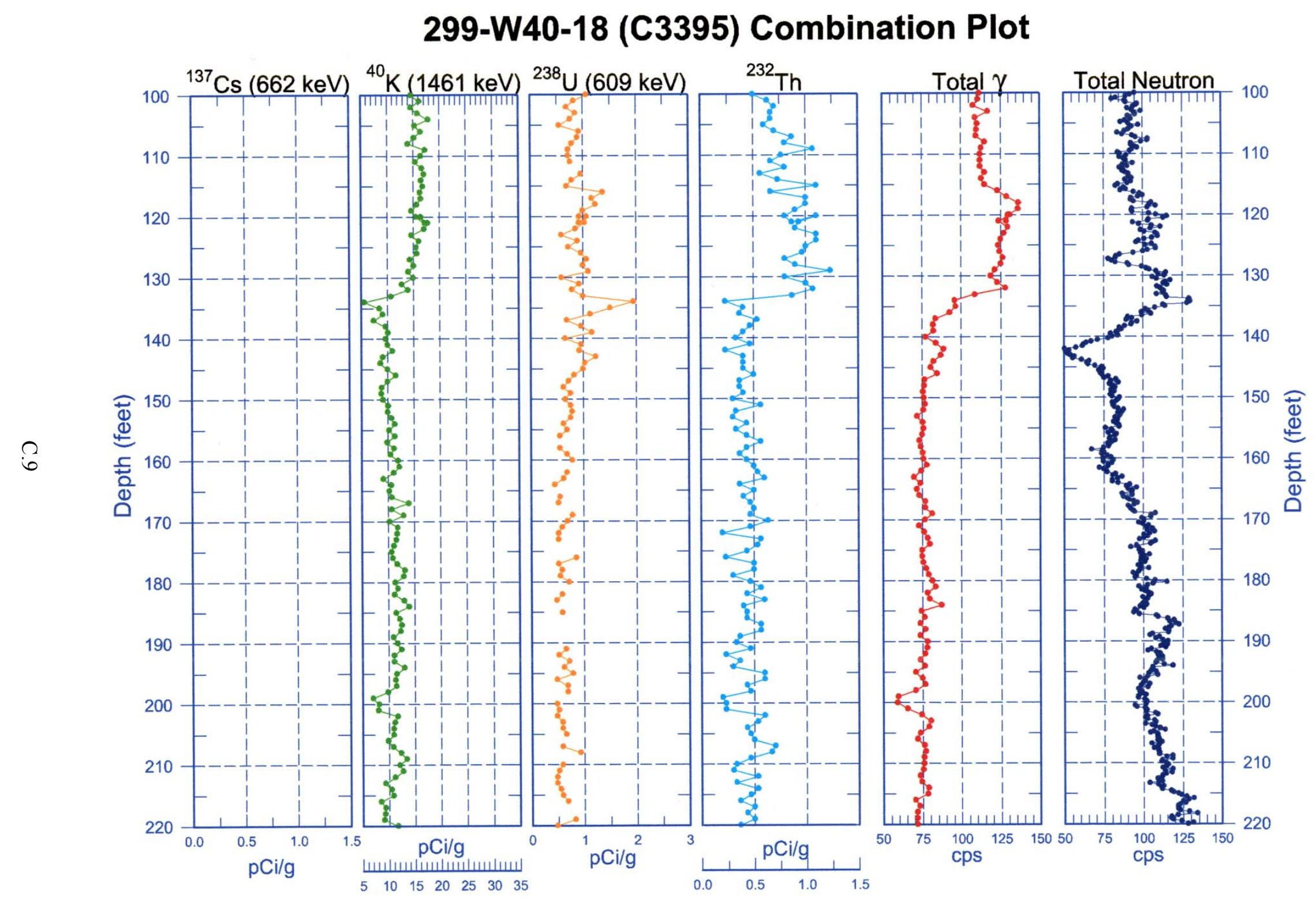


299-W40-18 (C3395) Combination Plot

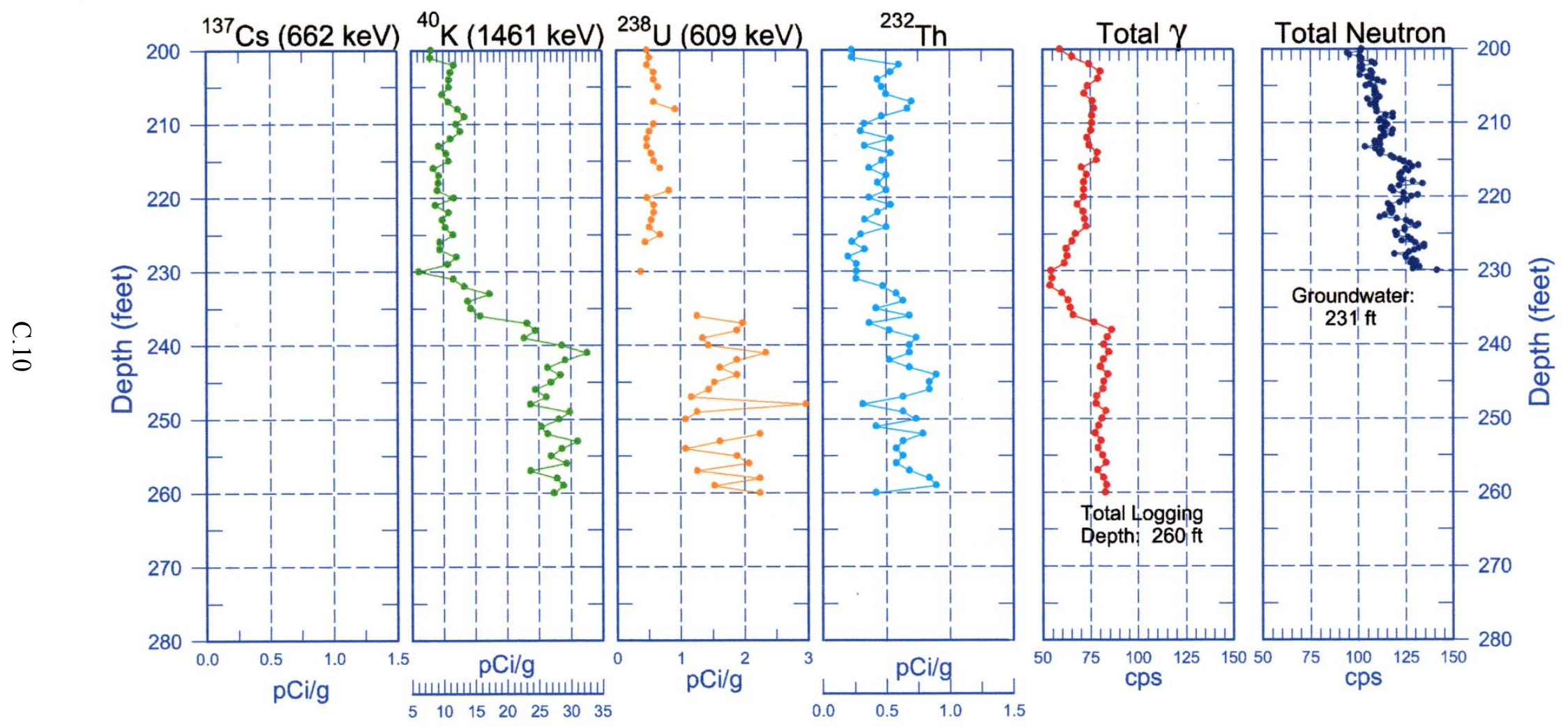




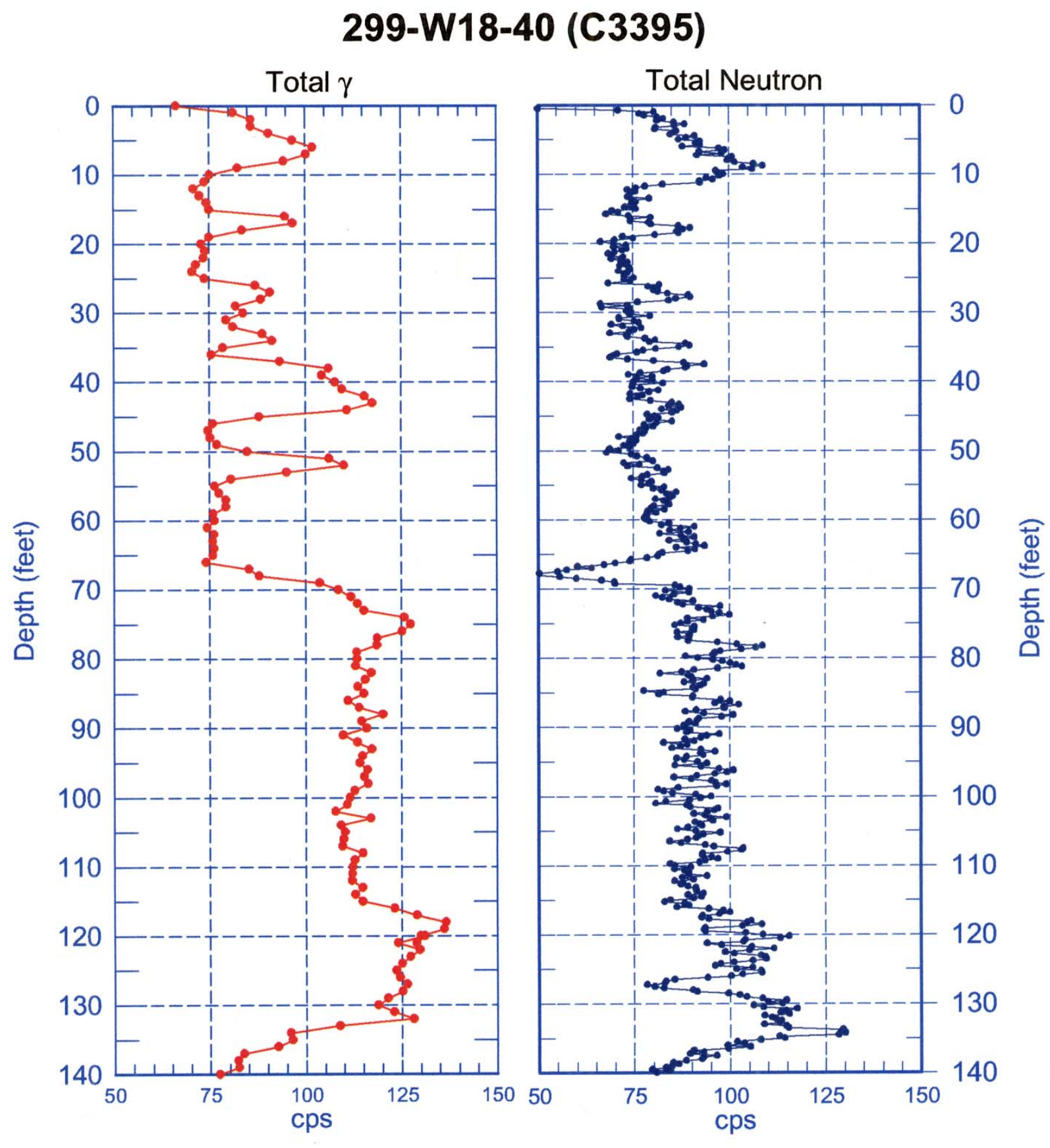

C. 11 


\section{9-W18-40 (C3395)}
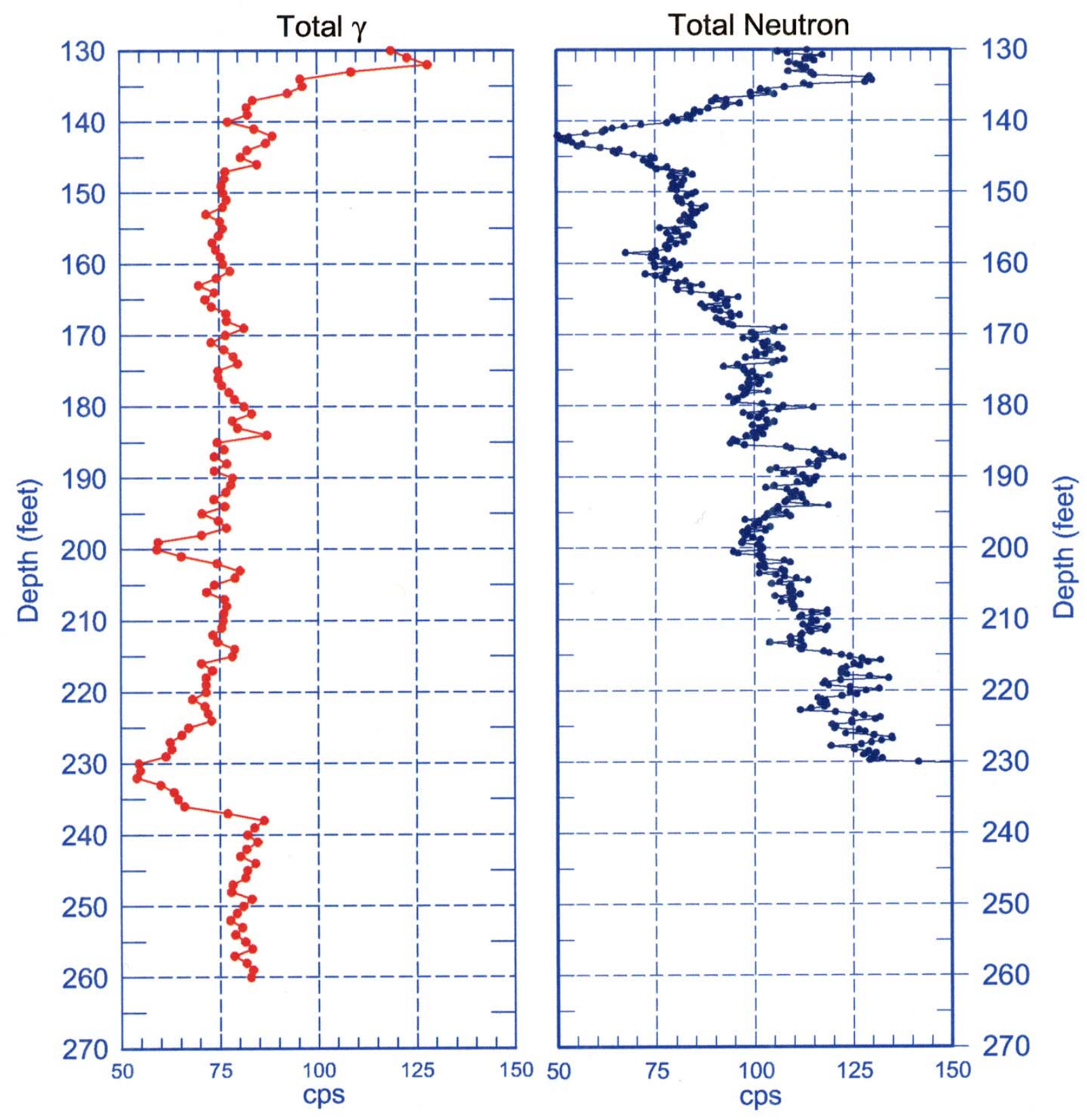

C.12 


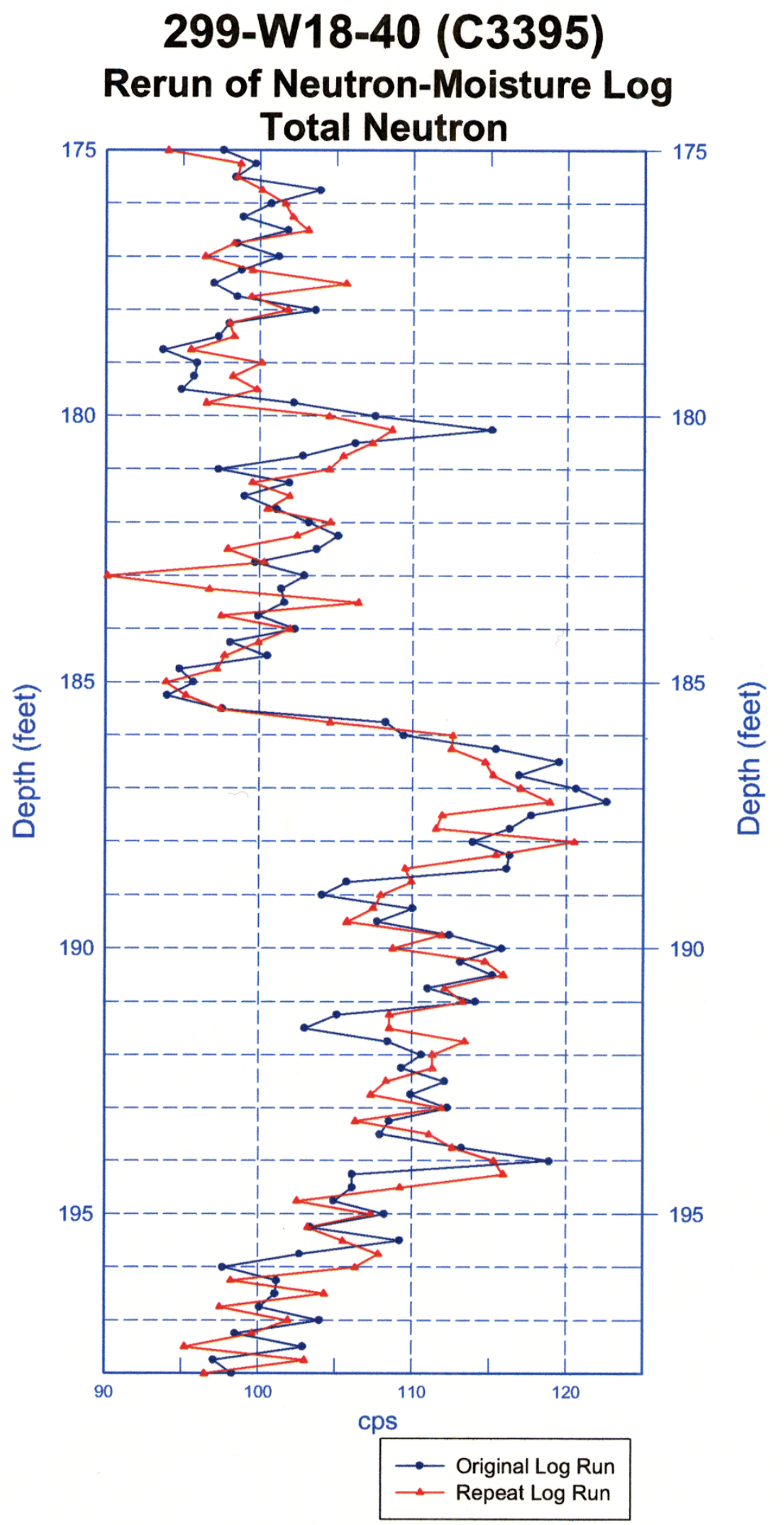

C. 13 


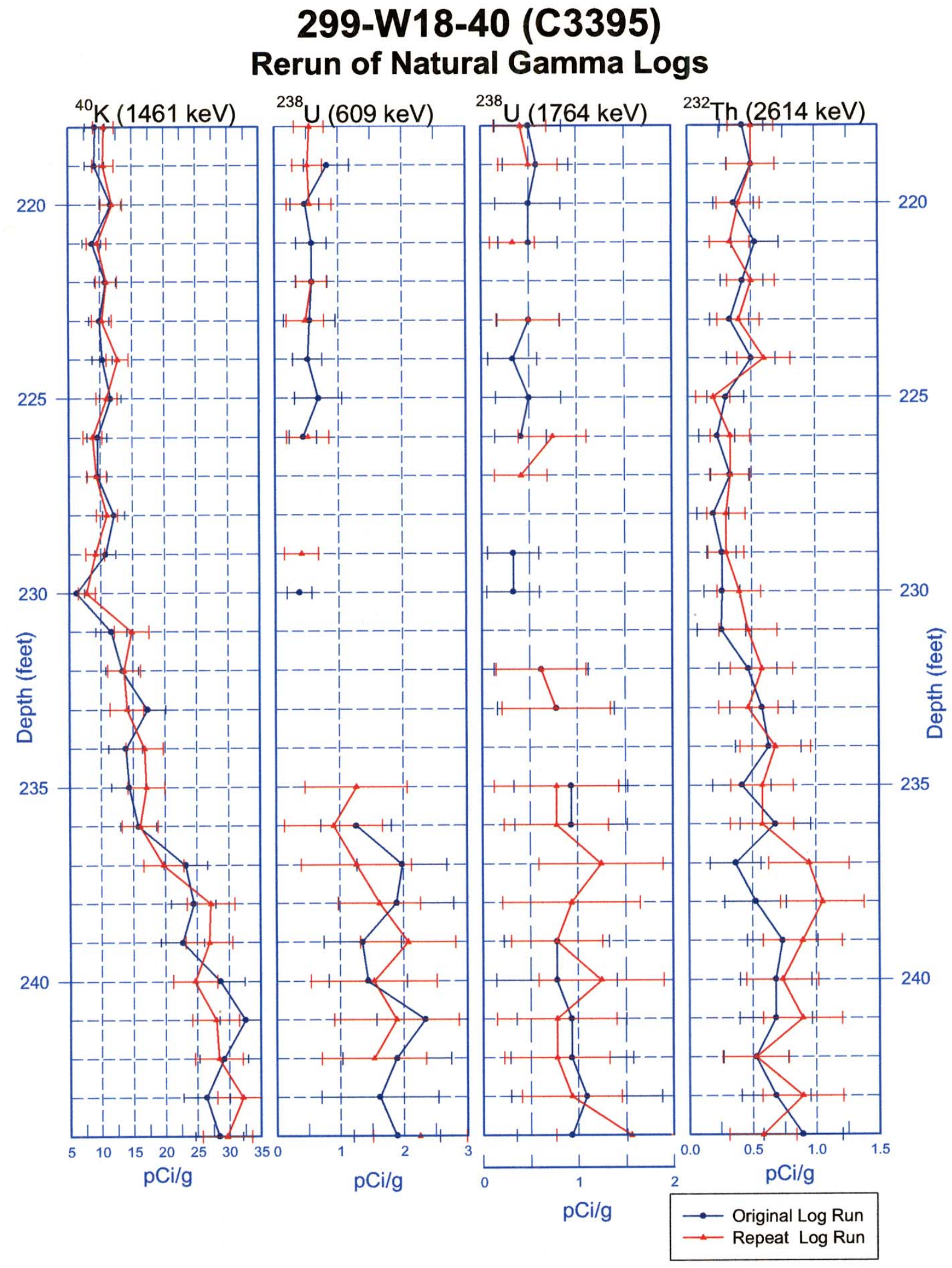

C. 14 


\section{9-W19-44 (C3393)}

\section{Log Data Report}

Borehole Information:

\begin{tabular}{|c|c|c|c|c|c|}
\hline \multirow{2}{*}{\multicolumn{2}{|c|}{ Borehole: 299-W1944 (C3393) }} & & \multicolumn{3}{|c|}{ Site: U Farm Perimeter } \\
\hline & & GWL (ft): & -230 & GWL Date: & 104 \\
\hline $\begin{array}{l}\text { North } \\
N^{3} A^{3}\end{array}$ & $\begin{array}{c}\text { East } \\
\text { NAA }\end{array}$ & $\begin{array}{c}\text { Drill Date } \\
\text { B/01 }\end{array}$ & $\begin{array}{c}\text { Toc Elevation } \\
\text { N/A }\end{array}$ & $\begin{array}{c}\text { Total Depth (fi) } \\
272\end{array}$ & $\begin{array}{l}\text { Type } \\
\text { Cable }\end{array}$ \\
\hline
\end{tabular}

\section{Casing Information:}

\begin{tabular}{|c|c|c|c|c|c|c|}
\hline Casing Type & Stickup (ft) & $\begin{array}{c}\text { Outer } \\
\text { Diameter } \\
\text { (in.) }\end{array}$ & $\begin{array}{c}\text { Inside } \\
\text { Diameter } \\
\text { (in.) }\end{array}$ & $\begin{array}{l}\text { Thickness } \\
\text { (in.) }\end{array}$ & $\begin{array}{l}\text { Top } \\
\text { (ft) }\end{array}$ & $\begin{array}{l}\text { Bottom } \\
\text { (ft) }\end{array}$ \\
\hline $\begin{array}{l}\text { Steel } \\
\text { Steel }\end{array}$ & $\begin{array}{c}0.6 \\
2.23\end{array}$ & $\begin{array}{c}11.75 \\
8.75\end{array}$ & $\begin{array}{c}10.25 \\
7.75\end{array}$ & $\begin{array}{c}0.75 \\
0.5\end{array}$ & $\begin{array}{l}0 \\
0\end{array}$ & $\begin{array}{c}61 \\
272\end{array}$ \\
\hline
\end{tabular}

\section{Borehole Notes:}

This borehole is a RCRA groundwater well. The logging engineer measured the casing stickup at the borehole using a steel tape and caliper. Explosive environment (high hydrogen) exists at this borehole per Tim Hottle (BHI). The inside diameter of the 12-inch casing could not be measured because of interference from the 8-inch casing. Very windy weather and much dust were experienced during logging in the late PM. SGLS logging detected groundwater below $231 \mathrm{ft}$.

\section{Logging Equipment Information:}

\begin{tabular}{|c|c|c|c|}
\hline Logging System: & Gamma 2B & & Type: SGLS (35\%) \\
\hline Calibration Date: & $09 / 00$ & Calibration Reference: & GJO-2001-245-TAR \\
\hline & & Logging Procedure: & MAC-HGLP 1.6.5 \\
\hline Logging System: & Gamma 2E & & Type: NMLS \\
\hline Callibration Date: & $05 / 01$ & Calibration Reference: & GJO-2001-247-TAR \\
\hline & & Logging Procedure: & MAC-HGLP 1.6.5 \\
\hline
\end{tabular}

\section{Spectral Gamma Logqing System (SGLS) Log Run Information:}

\begin{tabular}{|l|c|c|c|c|c|c|}
\hline Log Run & $\mathbf{1}$ & $\mathbf{2}$ & $\mathbf{3}$ & $\mathbf{4}$ & $\mathbf{5}$ & $\mathbf{6}$ \\
\hline Date & $9 / 04 / 01$ & $9 / 04 / 01$ & $9 / 04 / 01$ & $9 / 05 / 01$ & $9 / 05 / 01$ & \\
\hline Logging Engineer & Musial & Musial & Musial & Musial & Musial & \\
\hline Start Depth (ft) & 0 & 135.0 & 245.0 & 244.0 & 250.0 & \\
\hline Finish Depth (ft) & 135.0 & 245.0 & 225.0 & 270.0 & 255.0 & \\
\hline Count Time (sec) & 200 & 200 & 200 & 200 & 200 & \\
\hline Live/Real & $\mathrm{R}$ & $\mathrm{R}$ & $\mathrm{R}$ & $\mathrm{R}$ & $\mathrm{R}$ & \\
\hline Shield (Y/N) & $\mathrm{N}$ & $\mathrm{N}$ & $\mathrm{N}$ & $\mathrm{N}$ & $\mathrm{N}$ & \\
\hline MSA Interval (ft) & 1.0 & 1.0 & 1.0 & 1.0 & 1.0 & \\
\hline ft/min & na $^{4}$ & $\mathrm{n} / \mathrm{a}$ & $\mathrm{n} / \mathrm{a}$ & $\mathrm{Na}$ & $\mathrm{n} / \mathrm{a}$ & \\
\hline Pre-Verification & B0043CAB & B0043CAB & B0043CAB & B0044CAB & BO044CAB & \\
\hline Start File & B0043000 & B0043136 & B0043247 & B0044000 & B0044027 & \\
\hline Finish File & B0043135 & B0043246 & B0043272 & B0044026 & B0044032 & \\
\hline Post-Verification & B0043CAA & B0043CAA & B0043CAA & B0044CAA & B0044CAA & \\
\hline
\end{tabular}


Neutron Moisture Logging System (NMLS) Log Run Information:

\begin{tabular}{|c|c|c|c|c|c|c|}
\hline Log Run & 1 & 2 & 3 & 4 & 5 & 6 \\
\hline Date & $9 / 05 / 01$ & $9 / 05 / 01$ & $9 / 05 / 01$ & $9 / 05 / 01$ & & \\
\hline Logging Engineer & Musial & Musial & Musial & Musial & & \\
\hline Start Depth (ft) & 60 & 144.5 & 182.75 & 150.0 & & \\
\hline Finish Depth (ft) & 145.0 & 182.75 & 230.86 & 160.0 & & \\
\hline Count Time (sec) & na & na & 15 & na & & \\
\hline Live/Real & na & na & $\overline{\mathrm{R}}$ & na & & \\
\hline Shield $(Y / N)$ & $\mathbf{N}$ & $\mathbf{N}$ & $\bar{N}$ & $\mathbf{N}$ & & \\
\hline MSA Interval (ft) & 0.25 & 0.25 & 0.25 & 0.25 & & \\
\hline $\mathrm{ft} / \mathrm{min}$ & 1.0 & 1.0 & ná & 1.0 & & \\
\hline Pre-Verification & C0014CAB & C0014CAB & C0014CAB & C0014CAB & & \\
\hline Start File & C0014000 & C0014341 & C0015495 & $\mathrm{C} 0016000$ & & \\
\hline Finish File & $\mathrm{C0014340}$ & $\mathrm{C} 0014494$ & C0015687 & C0016040 & & \\
\hline Post-Verification & C0016CAA & $\overline{\mathrm{C}} 001 \overline{\mathrm{CAA}}$ & C0016CAA & C0016CAA & & \\
\hline
\end{tabular}

\section{Logging Operation Notes:}

A longer count time ( $200 \mathrm{sec})$ was required with the SGLS because of the relatively thick casing. In order to obtain reliable spectra while minimizing overail logging time, the depth interval was increased from 0.5 to $1.0 \mathrm{ft}$.

Log depths are relative to ground level.

The pre-run verification $\mathrm{B} 0043 \mathrm{CAB}$ file passed the verification criteria. Fine gain adjustment at $78.0 \mathrm{ft}$ (file $\mathrm{B} 0043078$ ).

Fine gain adjustment at $103.0 \mathrm{ft}$ (file B0043103).

Fine gain adjustment at $135.0 \mathrm{ft}$ (file B0043136).

The pre-nun verification B0044CAB file passed the verification criteria.

The neutron moisture tool was run centralized.

\section{Analysis Notes:}

\begin{tabular}{|c|c|c|c|c|c|}
\hline Analyst: & Sobczyk & Date: & $09 / 11 / 01$ & Reference: & MAC-VZCP 1.7.9, Rev. 2 \\
\hline
\end{tabular}

Pre-run and post-run verification spectra for the SGLS were evaluated. All of the pre-survey and postsurvey verification spectra were within the control limits. The post-survey verification spectra for all logging runs (files B0043CAA and B00044CAA) were outside of the lower warning limits for the peak counts per second at 609,1461 , and $2615 \mathrm{keV}$. Examinations of spectra indicate that the detector appears to have functioned normally during the log run. Individual spectra were processed in batch mode using APTEC SUPERVISOR to identify individual energy peaks and determine count rates. Concentrations were calculated with EXCEL. Corrections were applied for a casing thickness of 1.25 inches from the ground surface to $60 \mathrm{ft}$ and 0.5 inch from 61 to $270 \mathrm{ft}$. A correction for water in the borehole was applied at and below $231 \mathrm{ft}$. Dead time corrections were not necessary. The first and second reruns of the SGLS show good repeatability.

Pre-run and post-run verification spectra for the NMLS were evaluated. The pre -survey verification spectrum recorded 711 gross cps, while the post-survey verification spectrum recorded 799 gross cps.

Moisture calibration models at Hanford for 8-inch-diameter casing with 0.322 -inch thickness have been established. A casing thickness correction (relative to 8-inch casing) can be estimated. Thus, corrections were applied to the gross neutron counts per second to estimate volumetric moisture content with the established 8 -inch hole-size correction and the 0.5 -inch casing thickness for 8 -inch-diameter casing. Neutron data are also presented as gross counts and percent moisture by volume. In general, an increase in 
neutron count is indicative of an increase in moisture content. The rerun of the neutron-moisture tool shows good repeatability.

\section{Log Plot Notes:}

Separate log plots are provided for gross gamma, naturally occurring radionuclides $\left({ }^{40} \mathrm{~K},{ }^{232} \mathrm{Th},{ }^{238} \mathrm{U}\right.$, and associated decay progeny), and man-made radionuclides. For each radionuclide, the energy value of the spectral peak used for quantification is indicated. Unless otherwise noted, all radionuclides are plotted in picocuries per gram (pCi/g). ${ }^{238} \mathrm{U}$ activity based on both the 609 - and $1764-\mathrm{keV}$ photopeaks are plotted. The open circles indicate the minimum detectable activity (MDA) for each radionuclide. Error bars on each plot represent error associated with counting statistics only and does not include errors associated with the inverse efficiency function, dead time correction, or casing and water corrections. These errors are discussed in the calibration report. A combination plot is also included to facilitate correlation. A neutron moisture $\log$ of percent moisture by volume is also shown on the combination plot.

\section{Results and Interpretations:}

${ }^{137} \mathrm{Cs}$ was the only man-made radionuclide detected. ${ }^{137} \mathrm{Cs}$ activity was detected at a log depth of $3.0 \mathrm{ft}$. The measured ${ }^{137} \mathrm{Cs}$ activity was $0.4 \mathrm{pCi} / \mathrm{g}$ and is interpreted as surface contamination. A marginal peak at $1408 \mathrm{keV}$ was observed at $192 \mathrm{ft}$ (file $\mathrm{B} 0043193$ ); this peak is interpreted as the ${ }^{238} \mathrm{U}\left({ }^{214} \mathrm{Bi}\right) 1407.98-\mathrm{keV}$ peak, not the ${ }^{152} \mathrm{Eu} 1408.01-\mathrm{keV}$ peak, because vittually no contamination was detected in this borehole.

The large apparent increase in total gamma counts per second at $61 \mathrm{ft}$ is due to the change from a double to single string of casing at $61 \mathrm{ft}$. The casing correction for two strings of casing was applied from the ground surface to $60 \mathrm{ft}$ instead of $61 \mathrm{ft}$ because the log data appeared to be over corrected at $61 \mathrm{ft}$ if the correction for two casing strings were applied at $61 \mathrm{ft}$. Casing correction is not applied to gross gamma data, because the casing correction factor is energy dependent. The decrease in total gamma counts per second at $231 \mathrm{ft}$ is due to groundwater in the borehole shielding the detector. Changes in MDA at 60 and $231 \mathrm{ft}$ are due to changes in casing configuration and groundwater. The increase in apparent ${ }^{40} \mathrm{~K}$ activity from about 13 to 19 $\mathrm{pCi} / \mathrm{g}$ at about $51 \mathrm{ft}$ is interpreted as the top of the Hanford $\mathrm{H} 2$. The increase in ${ }^{232} \mathrm{Th}$ activity from about 0.8 to $1.2 \mathrm{pCi} / \mathrm{g}$ and the increase in gross gamma counts from 160 to $190 \mathrm{cps}$ at $130 \mathrm{ft}$ probably represents the top of the Early Palouse Soil. On the basis of low K-40 activities, the carbonate rich paleosols of the Pliocene-Pleistocene are interpreted as being between 144 and $147 \mathrm{ft}$. The caliche layer with characteristically high uranium content (greater than $1.5 \mathrm{pCi} / \mathrm{g}$ ) is present between 145 and $146 \mathrm{ft}$. The top of the Ringold is picked at $147 \mathrm{ft}$.

The neutron moisture tool's depressed response in this hole is due to the low-activity source and short source-to-detector spacing. The highest neutron counts occurred in the groundwater as expected. The elevated neutron counts per second that occur at about 129 through $147 \mathrm{ft}$ corresponds with the PlioPleistocene as interpreted from the SGLS data.

\footnotetext{
${ }^{1} \mathrm{GWL}$ - groundwater level

${ }^{2}$ TOC - top of casing

${ }^{3} \mathrm{~N} / \mathrm{A}$ - not available

${ }^{4} \mathrm{n} / \mathrm{a}$ - not applicable
} 


\section{9-W19-44 (C3393) \\ Man-Made Radionuclide Concentrations}

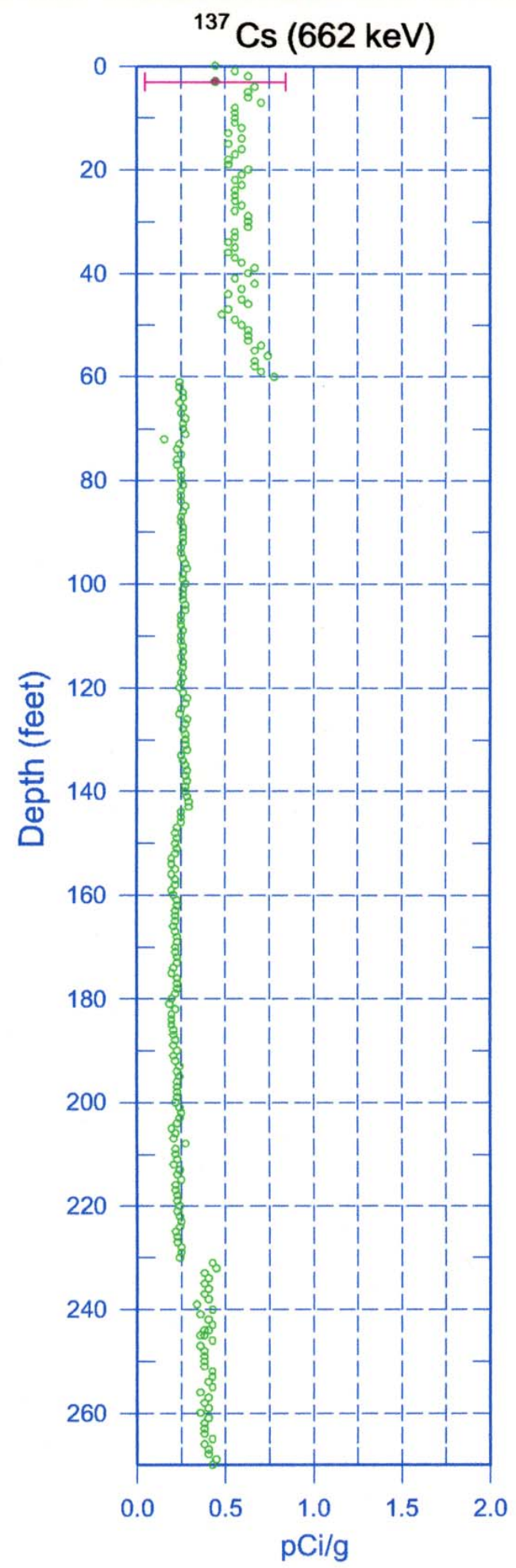




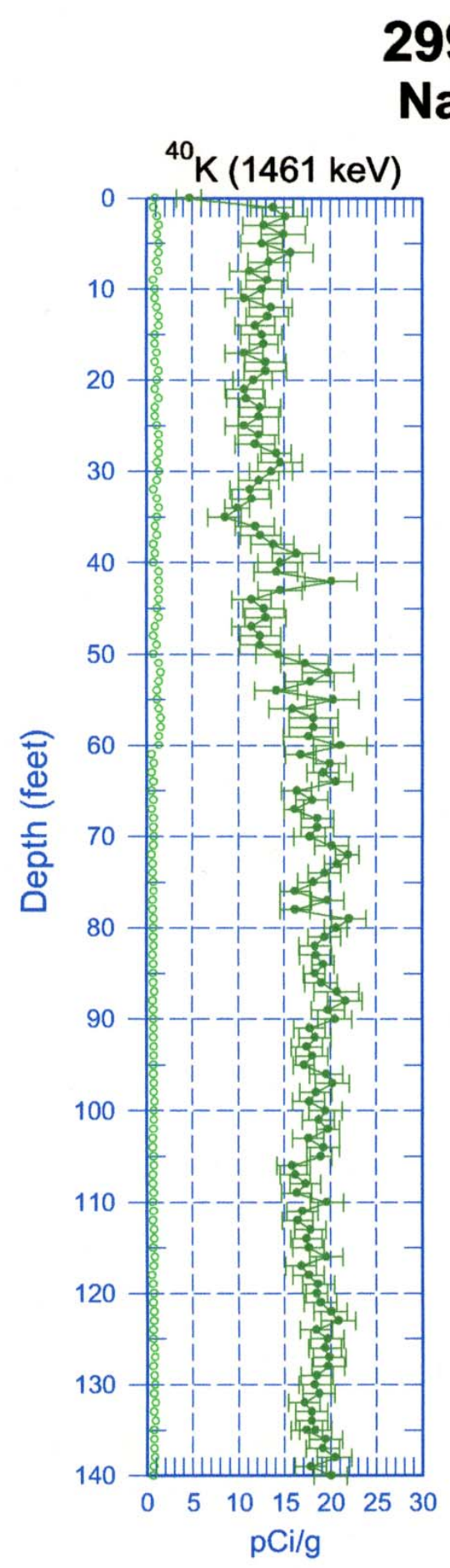

\section{9-W19-44 (C3393) Natural Gamma Logs}

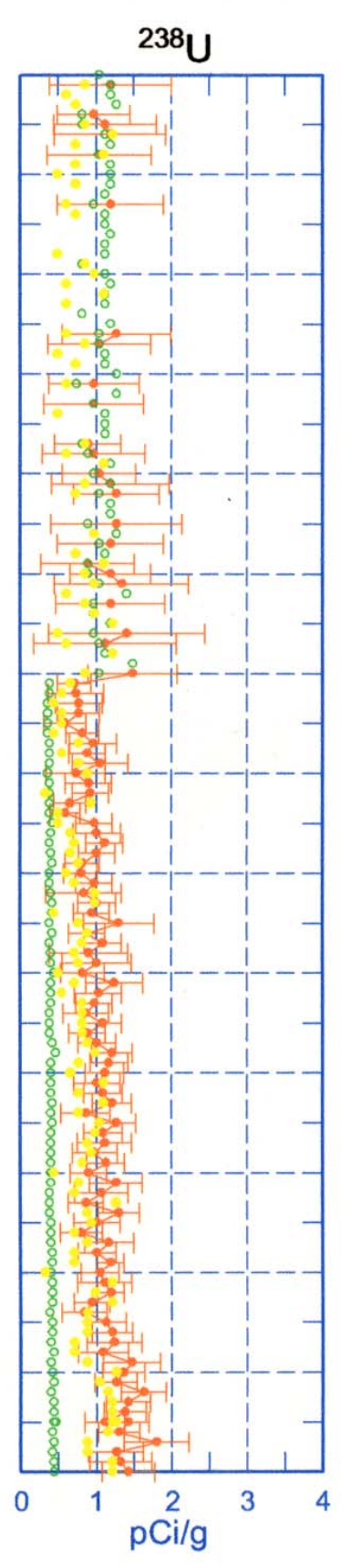

${ }^{232}$ Th (2614 keV)

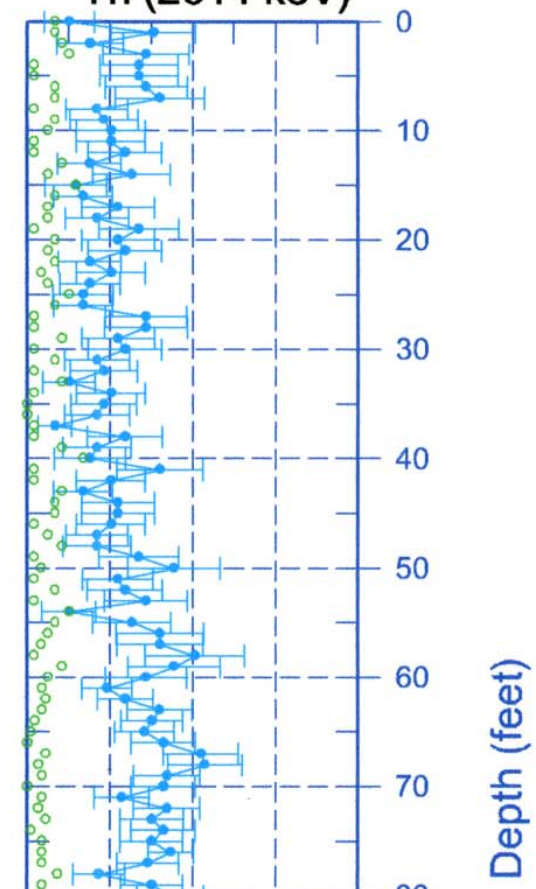

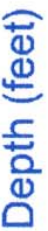




\section{9-W19-44 (C3393) \\ Natural Gamma Logs}
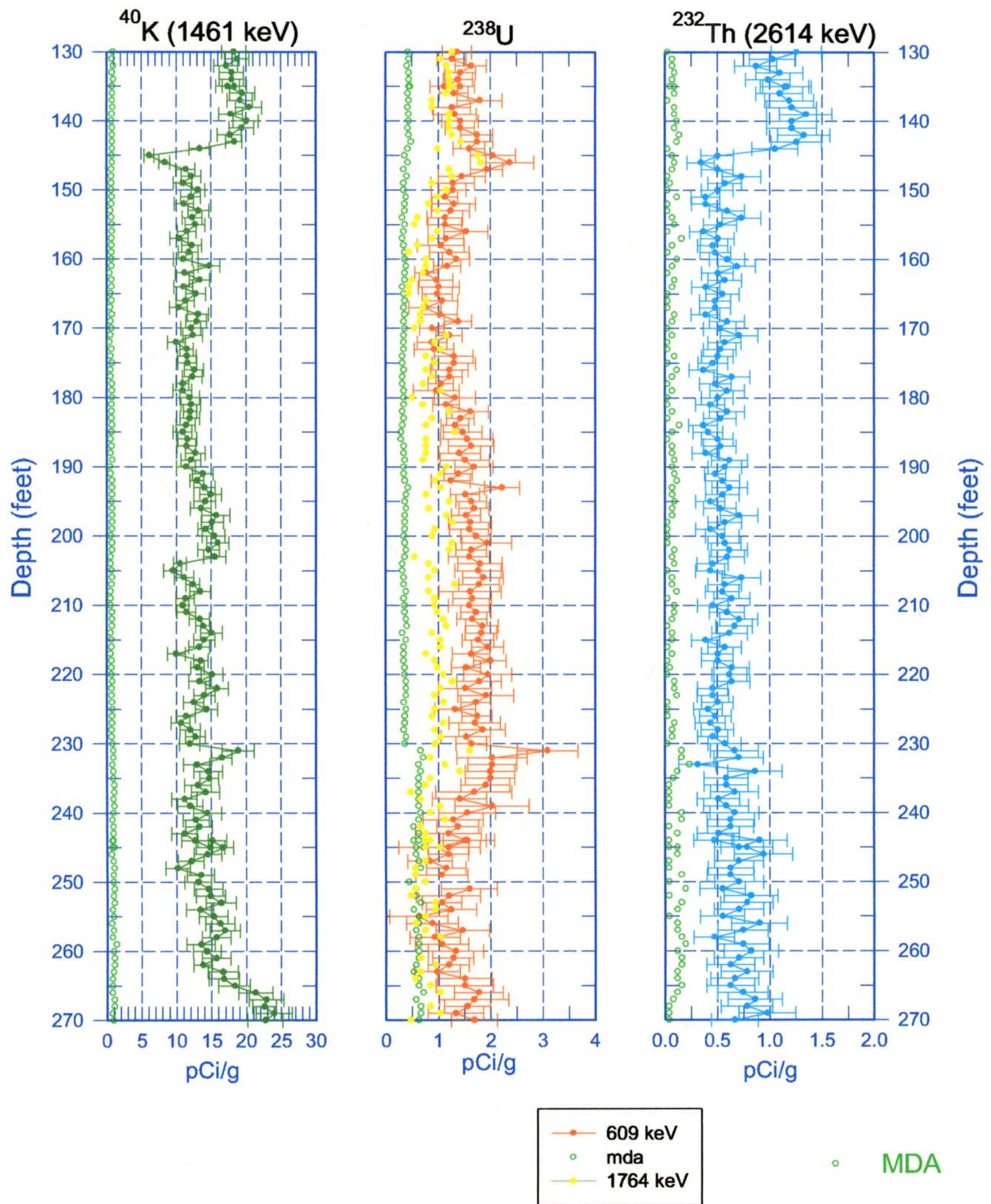

MDA 


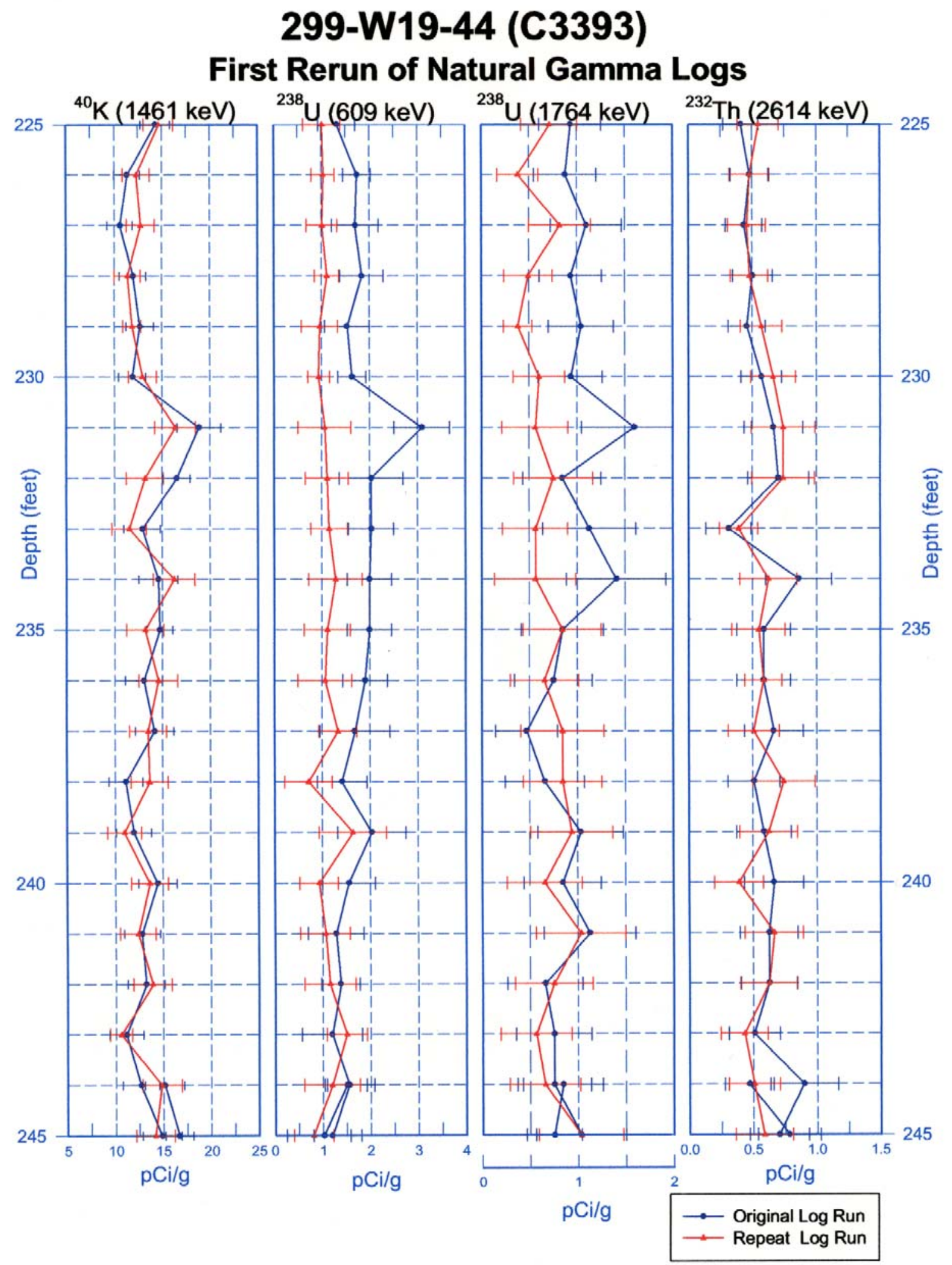

C. 21 


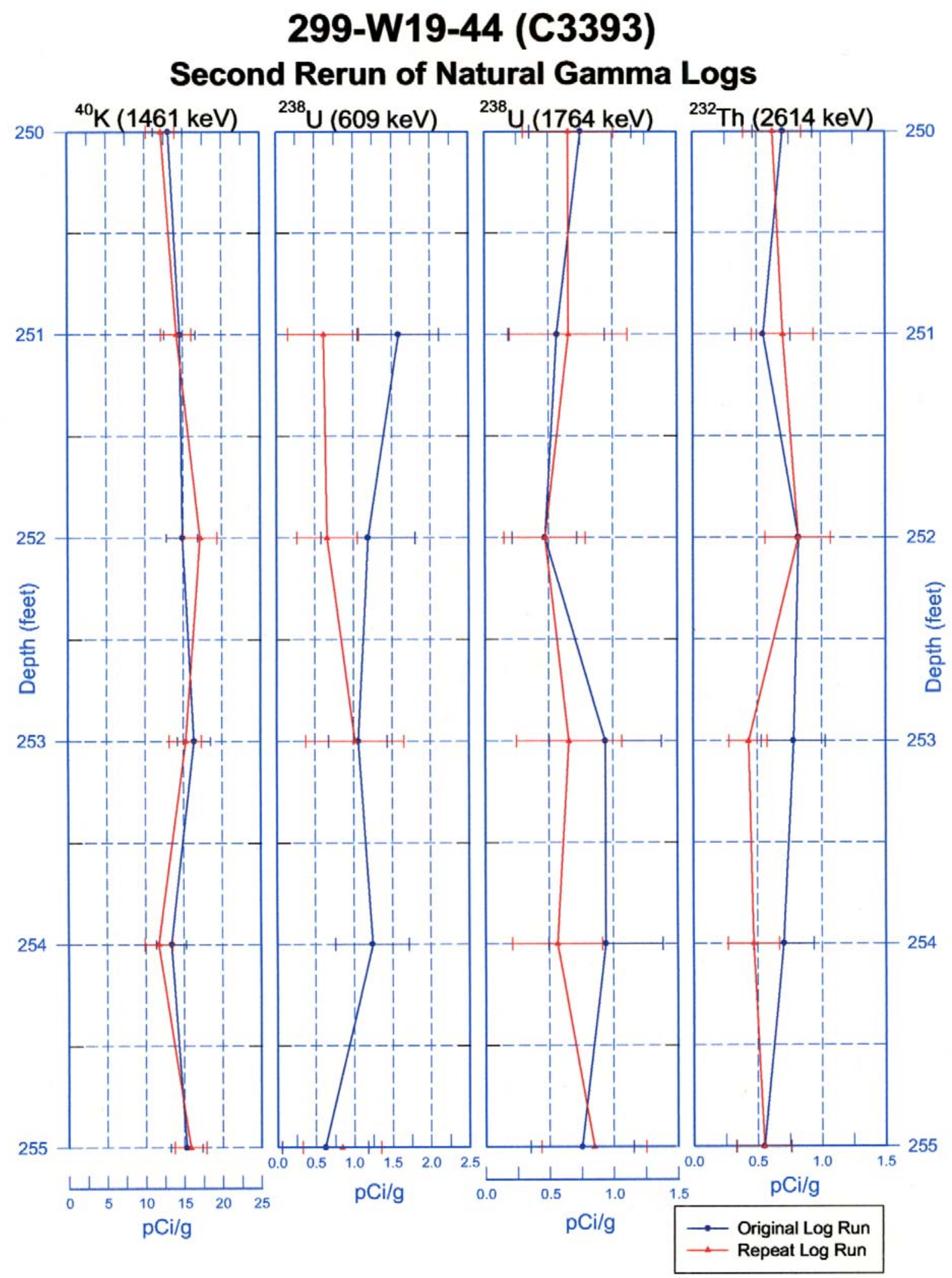

C. 22 


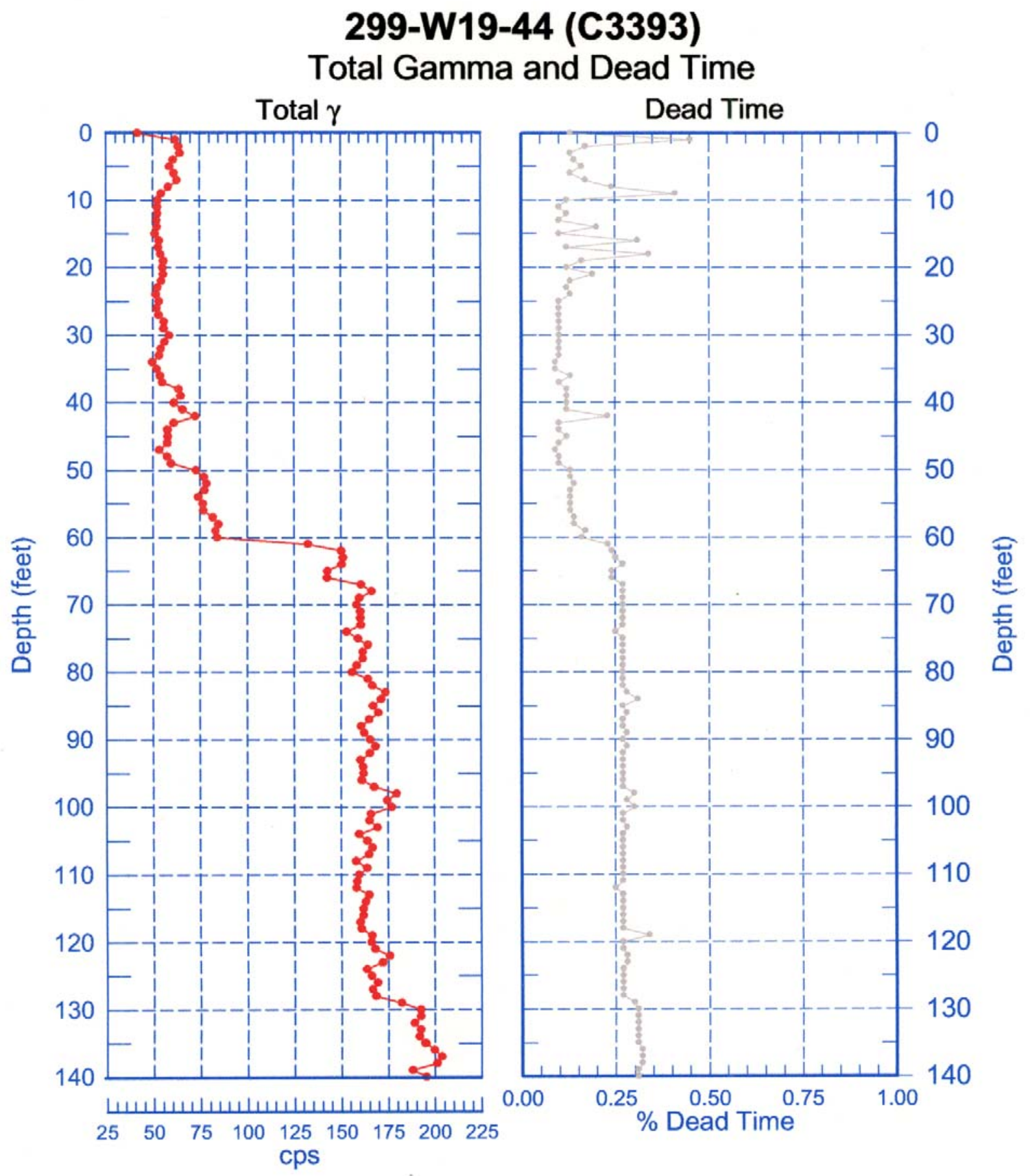




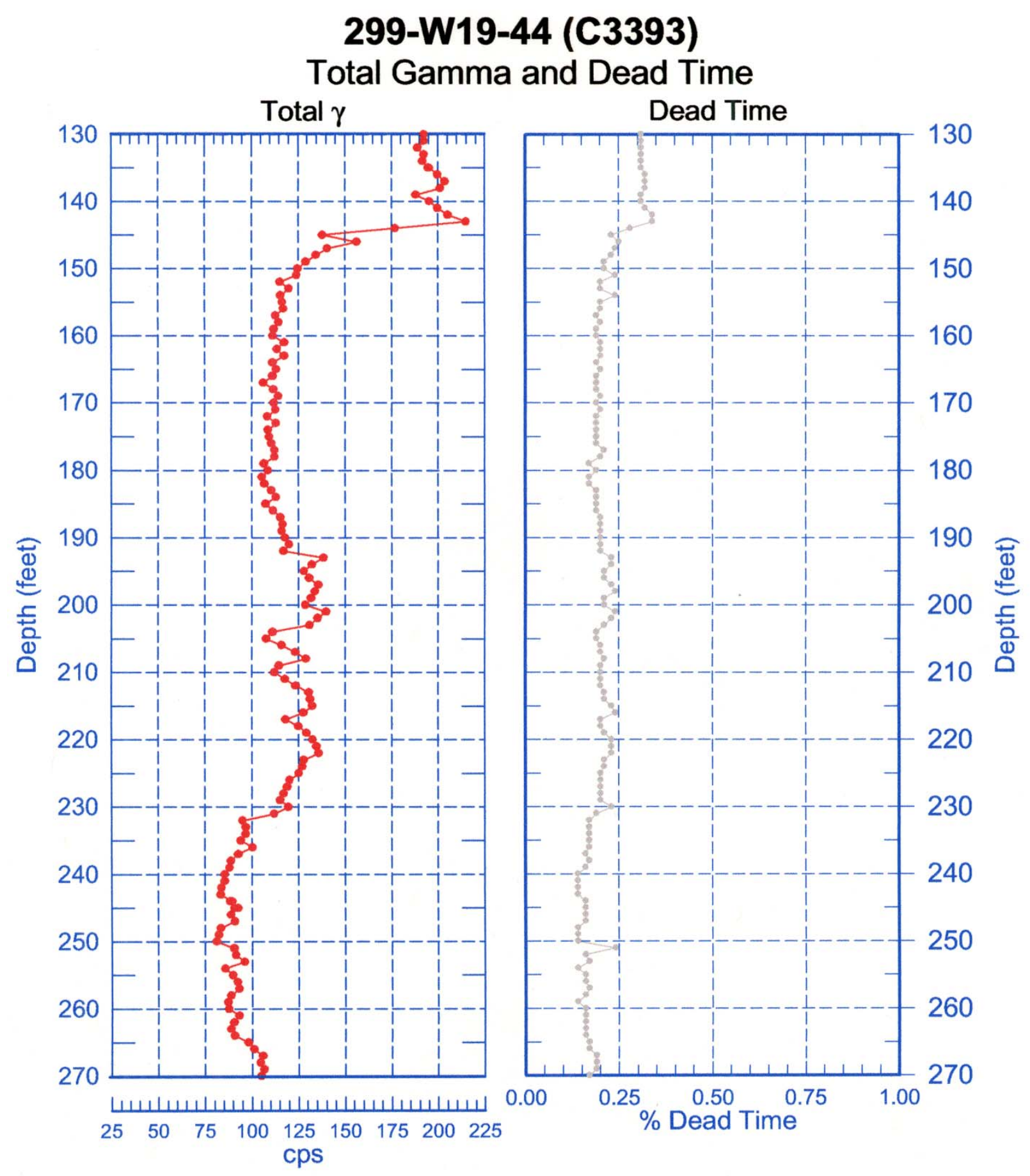




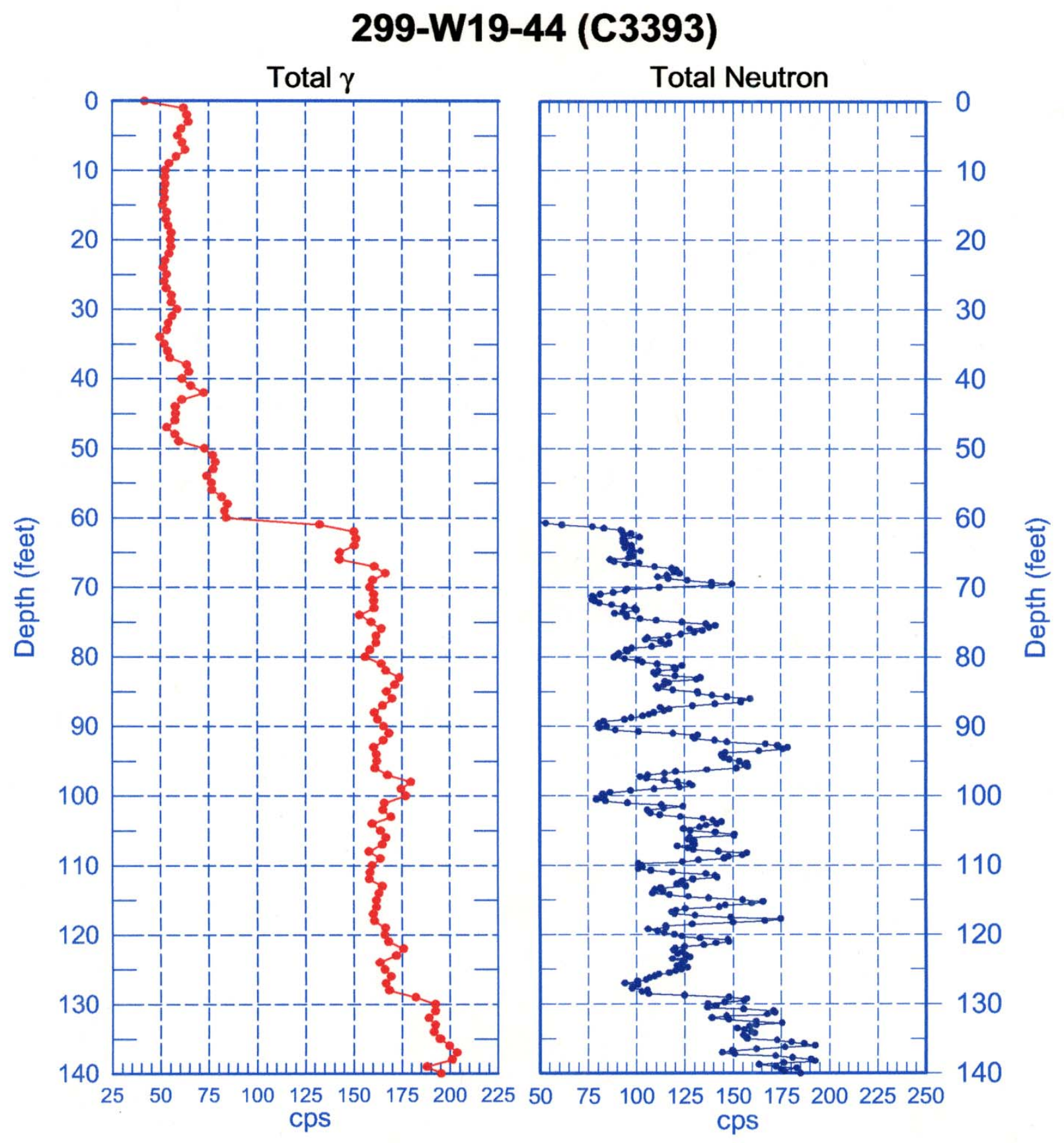




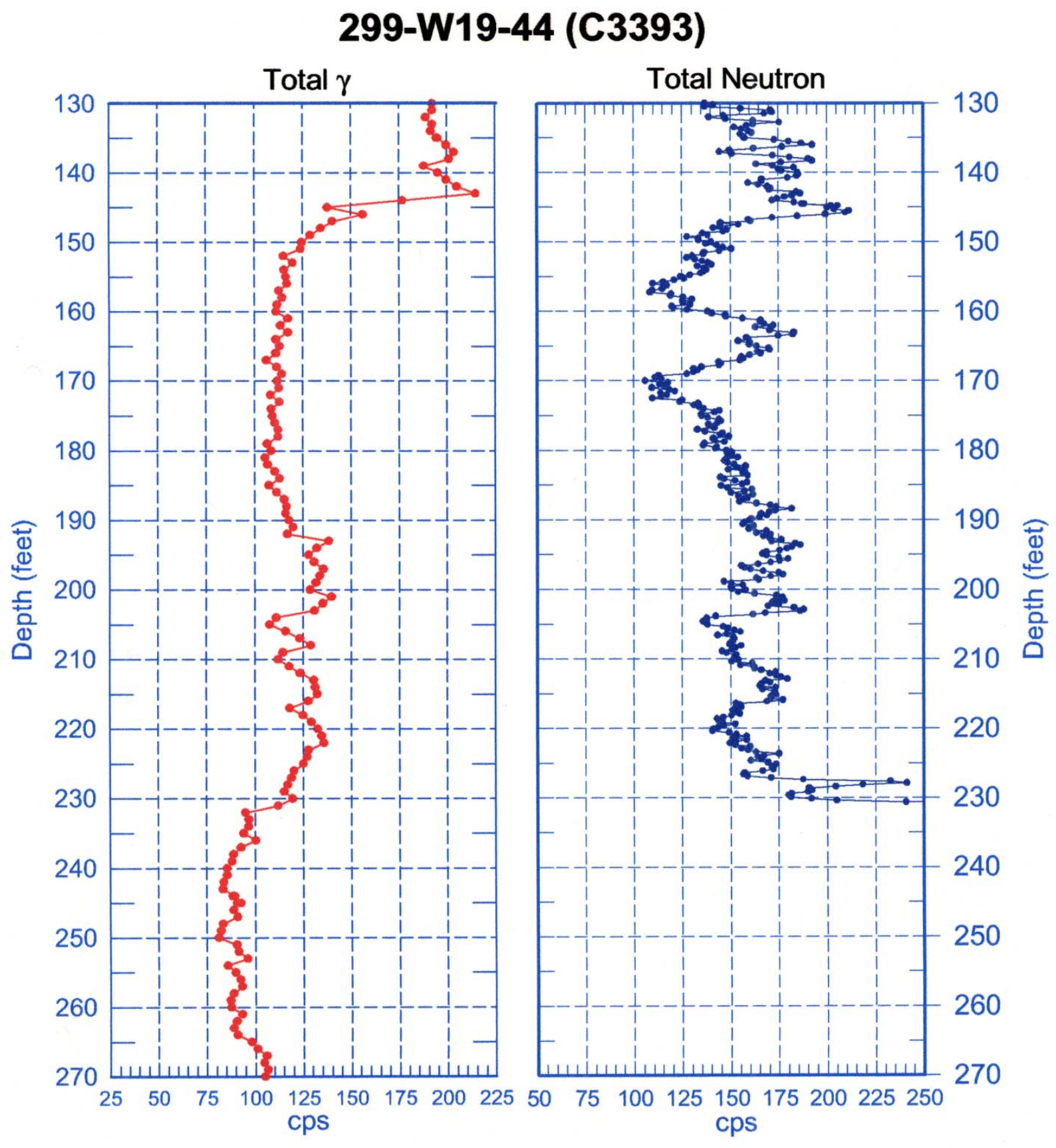




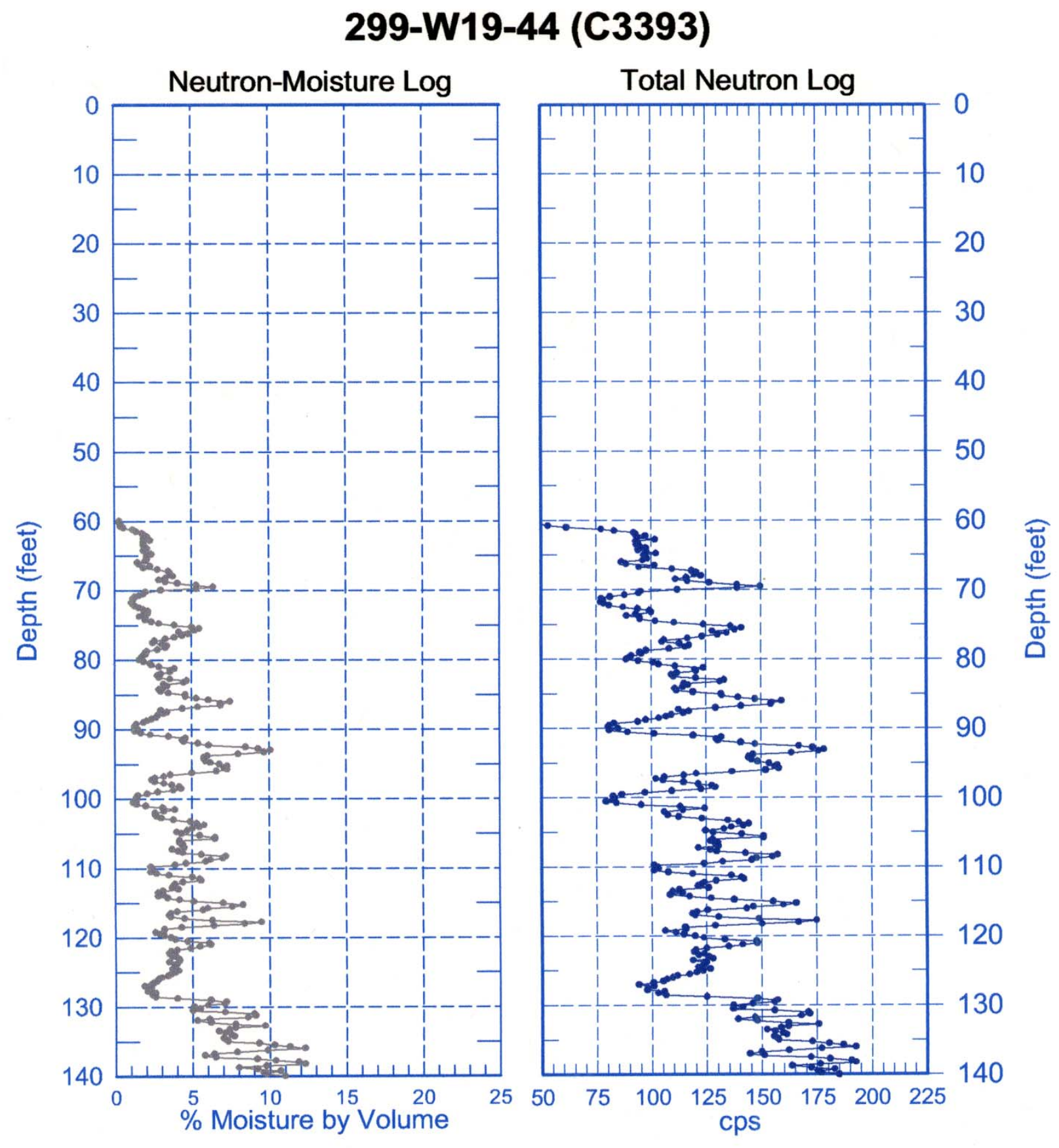




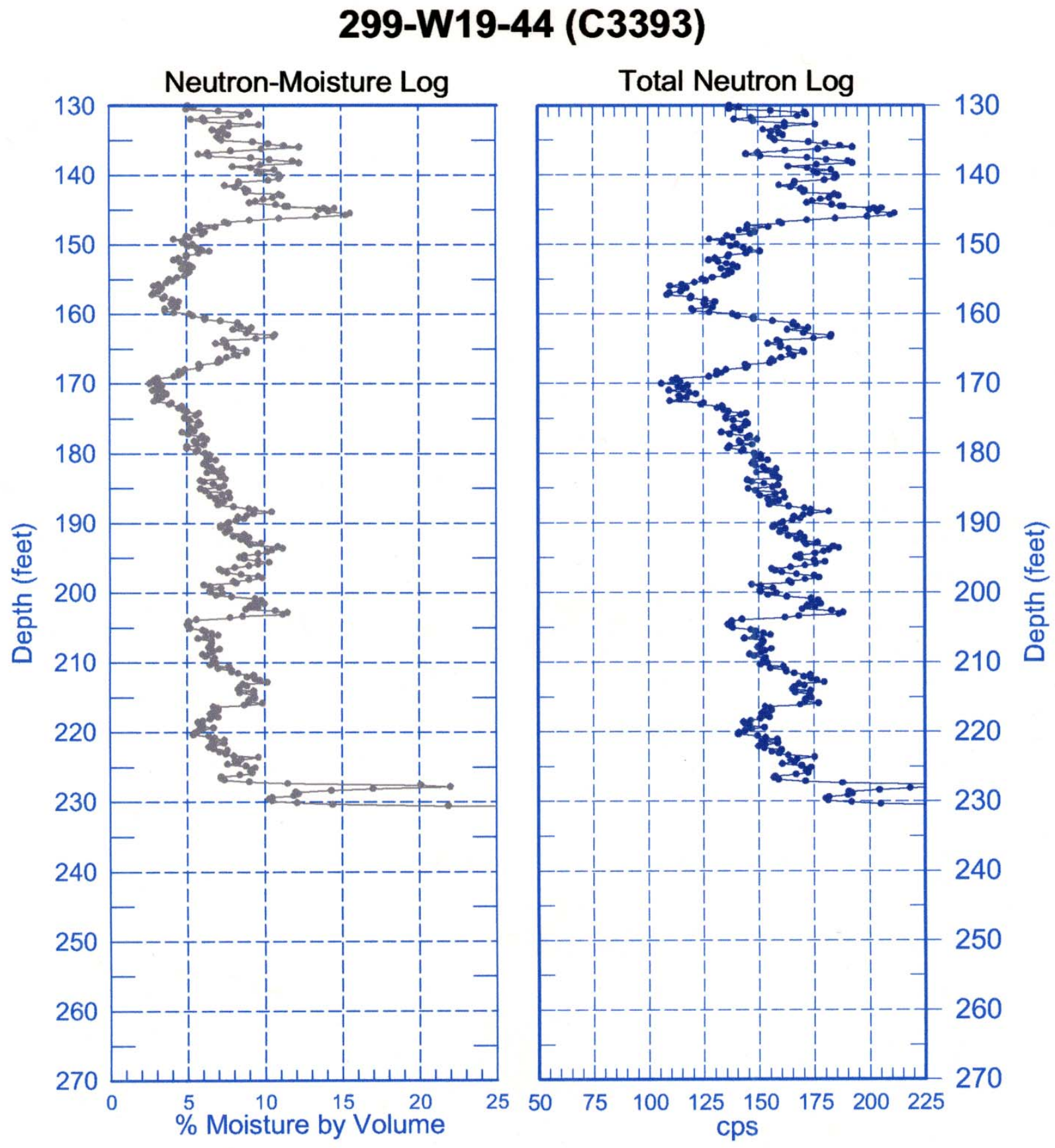

C. 28 


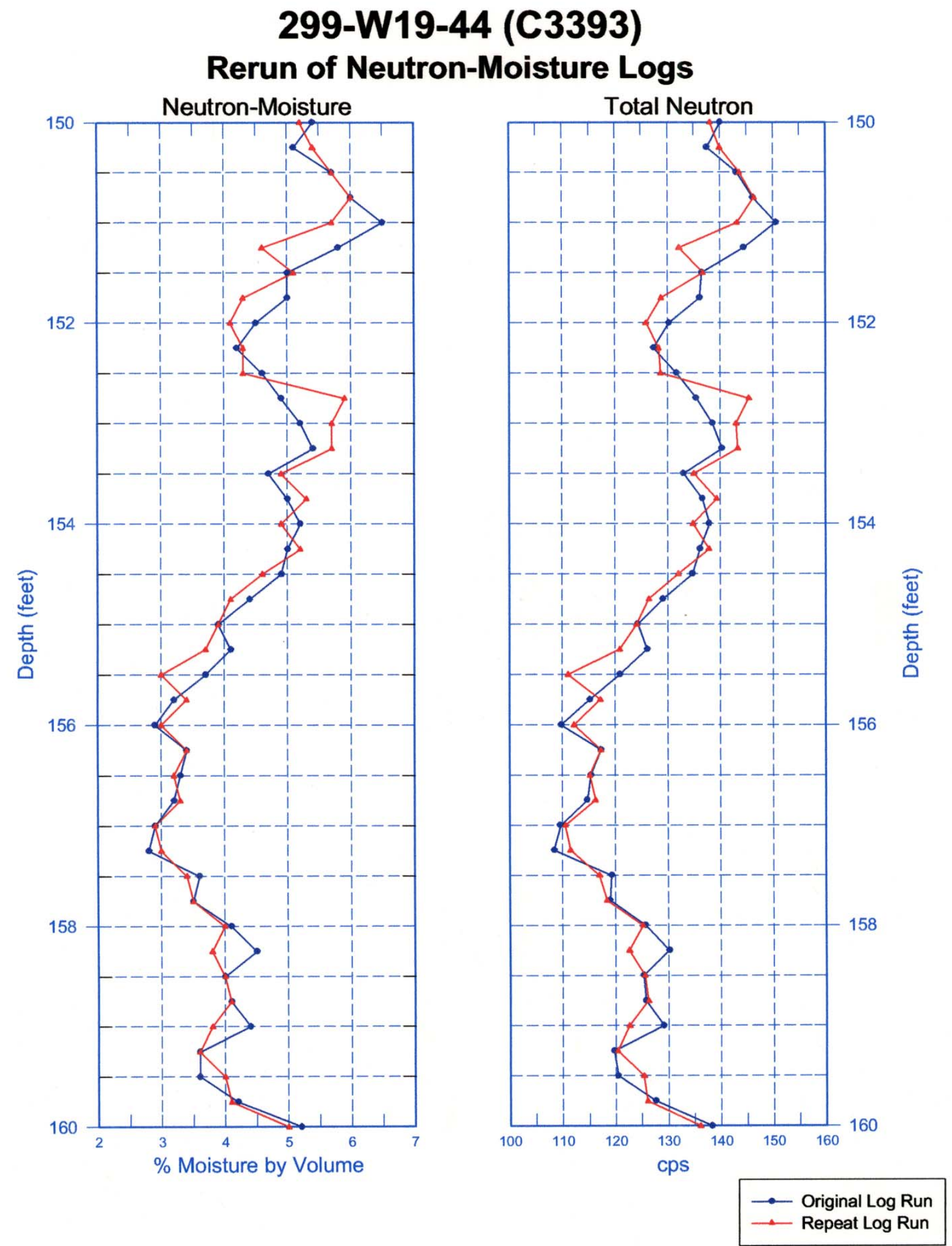

C. 29 


\section{9-W19-44 (C3393) Combination Plot}

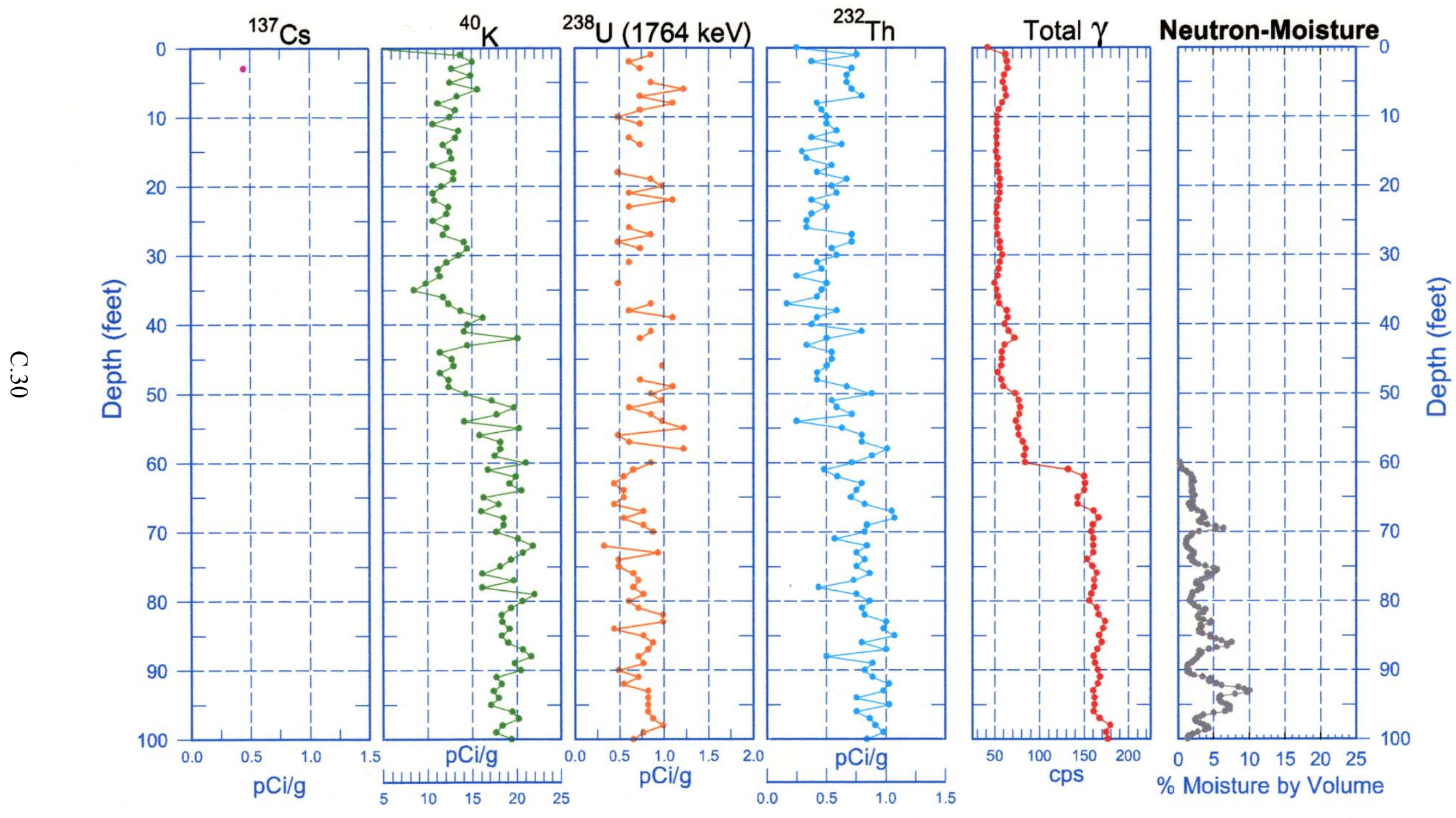




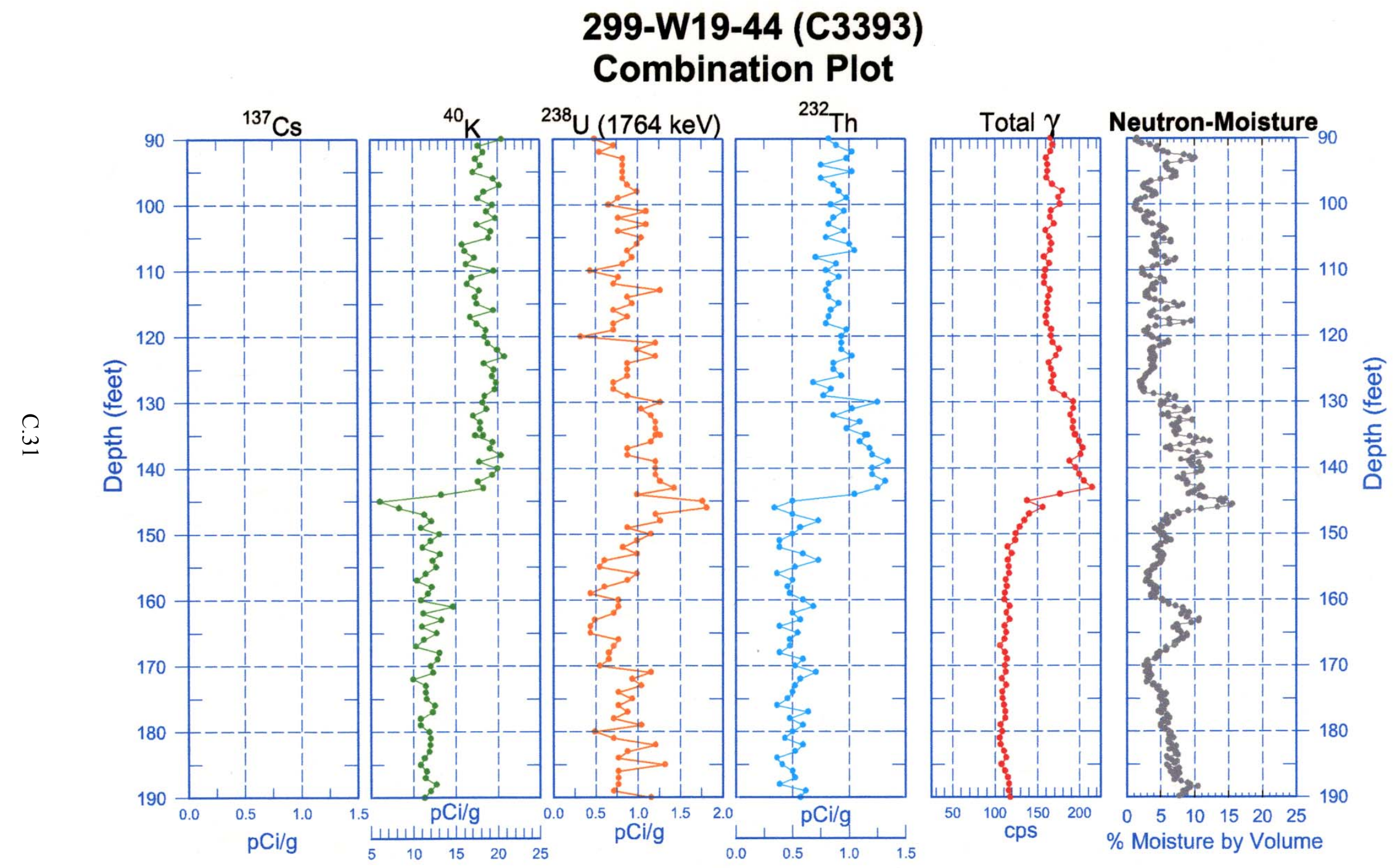




\section{9-W19-44 (C3393) Combination Plot}
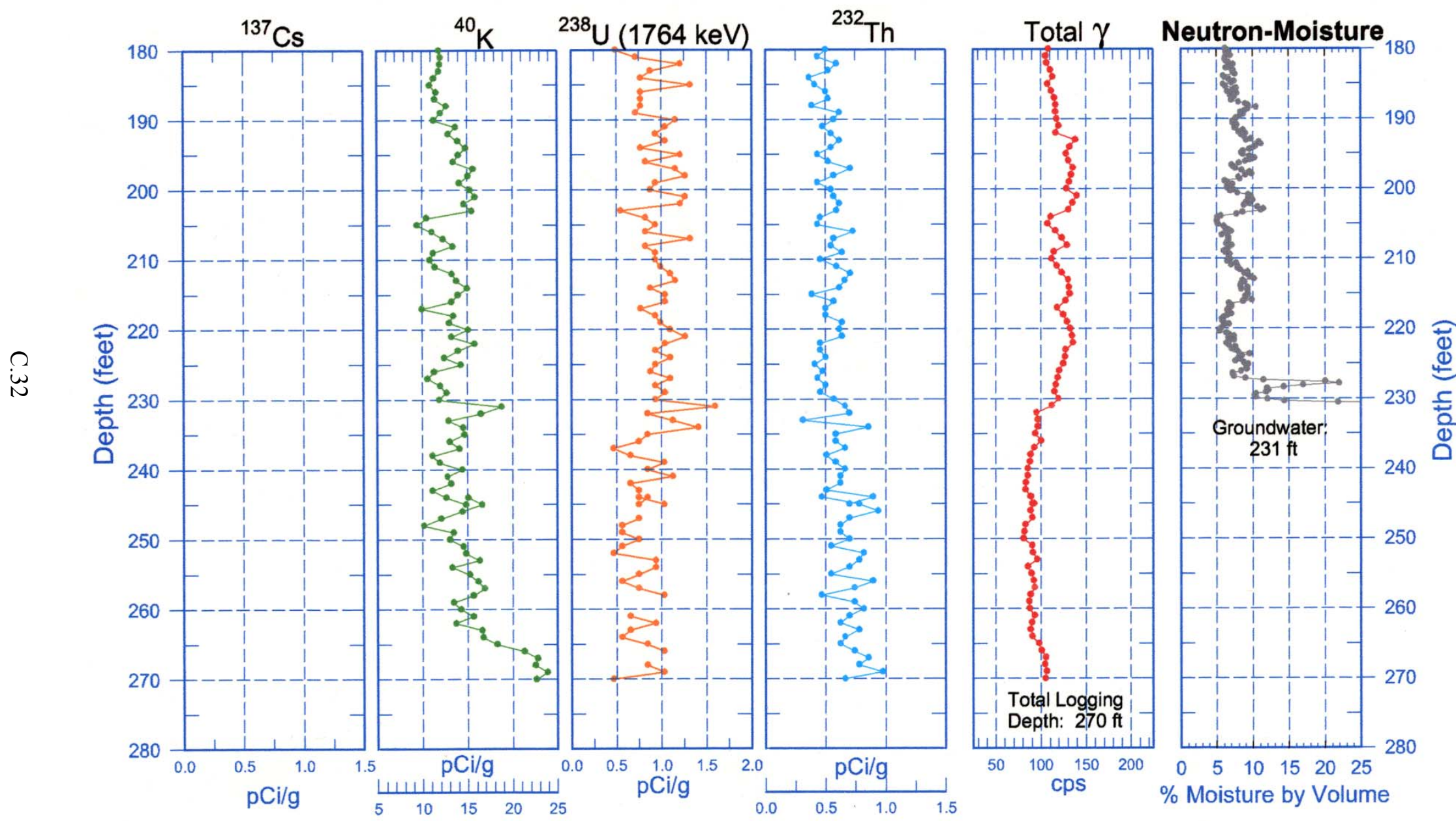


\section{9-W19-45 (C3394)}

\section{Log Data Report}

\section{Borehole Information:}

\begin{tabular}{|c|c|c|c|c|c|}
\hline \multicolumn{3}{|c|}{ Borehole: 299-W1945 (C3394) } & \multicolumn{3}{|c|}{ Site: U Farm Perimeter } \\
\hline & nates & GWL (fi): & -224.3 & GWL Date: & $8 / 15 / 01$ \\
\hline North & East & Drill Date & TOC Elevation & Total Depth (ft) & Type \\
\hline $\mathrm{N}^{3} \mathrm{~A}^{3}$ & N/A & $8 / 01$ & NA & 266.1 & air rotary \\
\hline
\end{tabular}

\section{Casing Information:}

\begin{tabular}{|l|c|c|c|c|c|c|}
\hline Casing Type & $\begin{array}{c}\text { Stickup } \\
\text { (ft) }\end{array}$ & $\begin{array}{c}\text { Outer } \\
\text { Diameter } \\
\text { (in.) }\end{array}$ & $\begin{array}{c}\text { Inside } \\
\text { Diameter } \\
\text { (in.) }\end{array}$ & $\begin{array}{c}\text { Thickness } \\
\text { (in.) }\end{array}$ & $\begin{array}{c}\text { Top } \\
\text { (fi) }\end{array}$ & $\begin{array}{c}\text { Bottom } \\
\text { (ft) }\end{array}$ \\
\hline Steel threaded drill pipe & 0.25 & $\mathbf{B . 2 5}$ & 7.25 & .75 & 0 & 266.1 \\
\hline & & & & & & \\
\hline
\end{tabular}

\section{Borehole Notes:}

This borehole is a RCRA groundwater well that was logged through the drill pipe.

\section{Logqing Equipment Information:}

\begin{tabular}{|c|c|c|}
\hline Logging System: & Gamma 2B & Type: SGLS (35\%) \\
\hline Callbration Date: & Calibration Reference: & GJO-2001-245-TAR \\
\hline & Logging Procedure: & MAC-HGLP 1.6.5 \\
\hline Logging System: & Gamma 2E & Type: NMLS \\
\hline Calibration Date: & Calibration Reference: & GJO-2001-247-TAR \\
\hline & Logging Procedure: & MACFGLP 1.6.5 \\
\hline
\end{tabular}

\section{Spectral Gamma Logging System (SGLS) Log Run Information:}

\begin{tabular}{|c|c|c|c|c|c|}
\hline Log Run & 1 & 2/Repeat & 3 & MRepeat & \\
\hline Date & $\mathrm{B} / 15 / 01$ & $8 / 15 / 01$ & $8 / 16 / 01$ & $8 / 16 / 01$ & \\
\hline Logging Engineer & Musial & Musial & Musial & Musial & \\
\hline Start Depth (ft) & 0 & 115 & 114 & 135 & \\
\hline Finish Depth (ft) & 115 & 103 & 266 & 150 & \\
\hline Count Time (sec) & 200 & 200 & 200 & 200 & \\
\hline Live/Real & $\mathbf{R}$ & $\mathbf{R}$ & $\mathbf{R}$ & $\mathrm{R}$ & \\
\hline Shield $(\mathrm{Y} / \mathrm{N})$ & $\mathbf{N}$ & $\mathbf{N}$ & $\mathbf{N}$ & $\mathbf{N}$ & \\
\hline MSA interval (fi) & 1.0 & 1.0 & 1.0 & 1.0 & \\
\hline ft/min & $n / a^{4}$ & $\mathrm{n} / \mathrm{a}$ & na & na & \\
\hline Pre-Verification & $\mathrm{B0} 0036 \mathrm{CAB}$ & B00036CAB & $\mathrm{B} 00037 \mathrm{CAB}$ & B00037CAB & \\
\hline Start File & B0036000 & B0036116 & $\mathrm{B} 0037000$ & B0037153 & \\
\hline Finish File & B0036115 & B0036128 & $\mathrm{B} 0037152$ & $B 0037168$ & \\
\hline Post-Verification & B00036CAA & $\mathrm{B} 00036 \mathrm{CAA}$ & B00037CAA & B00037CAA & \\
\hline
\end{tabular}




\section{Neutron Moisture Logging System (NMLS) Log Run Information:}

\begin{tabular}{|c|c|c|c|c|c|}
\hline Log Run & 1 & 2 & 3/Repeat & & \\
\hline Date & $8 / 16 / 01$ & $8 / 16 / 01$ & $8 / 16 / 0^{\prime}$ & & \\
\hline Logging Engineer & Musial & Musial & Musial & & \\
\hline Start Depth (ft) & 0 & 111.75 & 110 & & \\
\hline Finish Depth (ft) & 111.75 & 225 & 88 & & \\
\hline Count Time (sec) & $\mathrm{n} / \mathrm{a}$ & $n / a$ & n/a & & \\
\hline Live/Real & $n / a$ & $\mathrm{n} / \mathrm{a}$ & $n / a$ & & \\
\hline Shield (Y/N) & $\mathbf{N}$ & $\mathbf{N}$ & $\bar{N}$ & & \\
\hline MSA Interval (ft) & 0.25 & 0.25 & 0.25 & & \\
\hline $\mathrm{ft} / \mathrm{min}$ & 1.0 & 1.0 & 1.0 & & \\
\hline Pre-Verification & C0012CAB & $\mathrm{C} 0012 \mathrm{CAB}$ & C0012CAB & & \\
\hline Start File & $\mathrm{C0012000}$ & $\mathrm{C} 00 \overline{012448}$ & $\mathrm{C} 0012901$ & & \\
\hline Finish File & $\mathrm{C} 0012447$ & C0012900 & C0012989 & & \\
\hline Post-Venfication & C0012CAA & C0012CAA & C0012CAA & & \\
\hline
\end{tabular}

\section{Logging Operation Notes:}

A longer count time ( $200 \mathrm{sec}$ ) was required with the SGLS because of the relatively thick casing. The borehole was logged in the drill pipe before completion as a groundwater monitoring well. To obtain reliable spectra while minimizing overall logging time, the depth interval was increased ftom 0.5 to $1.0 \mathrm{ft}$.

SGLS log depths are relative to ground level. During logging runs, no fine gain adjustments occurted.

The pre-fun verification B0036CAA file passed the verification criteria. The post-survey verification $\mathrm{B} 0036 \mathrm{CAA}$ failed to meet the acceptance criteria. The counts of the 1460 and $2614 \mathrm{keV}$ peaks were both below the waming limits, and the counts of the $609-\mathrm{keV}$ peak were below the control limit. The tool, however, appears to be functioning normally. The counts of the 609 - and $1460-\mathrm{keV}$ peaks in the pre -survey verification B0037CAB were both below the warning limits, however, the tool appears to be functioning properly.

Neutron moisture logs were run on $8 / 16 / 01$ using the RLS 1 , and $\log$ depths are relative to ground level. The neutron moisture tool was run centralized.

\section{Analysis Notes:}

\begin{tabular}{|l|l|l|l|l|l|}
\hline Analyst: & Sobczyk & Date: & $08 / 28 / 01$ & Reference: & MAC-VZCP 1.7.9 Rev. 2 \\
\hline
\end{tabular}

Pre-run and post-run verification spectra for the SGLS were evaluated. All of the pre-survey verification spectra were within the control limits. The post-survey verification spectrum for logging run 1 (file B00036CAA) was the only post-survey verification spectrum that was outside of the control limits. The peak counts per second for the 609-keV peak was below the lower control limits for this post-run verification spectra. Examinations of spectra indicate that the detector appears to have functioned normally during the $\log$ run. Individual spectra were processed in batch mode using APTEC SUPERVISOR to identify individual energy peaks and deternine count rates. Concentrations were calculated with EXCEL. Corrections were applied for a casing thickness of 3/4 in. from the ground surface to $266 \mathrm{ft}$. A correction for water in the borehole was applied below $224 \mathrm{ft}$. Dead time corrections were not necessary.

Moisture calibration models at Hanford for 10 in. holes with 3/4-in, casing have not been established. Thus, the neutron $\log$ was not processed to estimate volumetric moisture content because the relatively large borehole diameter and casing thickness are beyond the range of conditions for which the tool was calibrated. Neutron data are presented as gross counts. In general, an increase in neutron count is 
indicative of an increase in moisture content, but a quantitative calculation of volumetric moisture cannot be made at this time.

Moisture calibration models at Hanford for 8-in. diameter casing with 0.322-in. thickness have been established. A casing thickness correction (relative to 8 -in. casing) can be estimated. Thus, corrections were applied to the gross neutron cps to estimate volumetric moisture content with the established 8 -in. hole -size correction and the $1 / 2$-inch casing thickness for 8 -in.-diameter casing. Neutron data are also presented as gross counts. In general, an increase in neutron count is indicative of an increase in moisture content.

The rerun of the neutron-moisture tool shows good repeatability, and the rerun may be off-depth by $-0.25 \mathrm{ft}$ compared to the original run.

\section{Log Plot Notes:}

Separate log plots are provided for gross gamma, naturally occurring radionuclides $\left({ }^{40} \mathrm{~K},{ }^{232} \mathrm{Th},{ }^{238} \mathrm{U}\right.$, and associated decay progeny), and man-made radionuclides. For each radionuclide, the energy value of the spectral peak used for quantification is indicated. Unless otherwise noted, all radionuclides are plotted in picocuries per gram ( $\mathrm{pCi} / \mathrm{g}$ ). The open circles indicate the minimum detectable activity (MDA) for each radionuclide. Error bars on each plot represent error associated with counting statistics only and does not include errors associated with the inverse efficiency function, dead time correction, or casing and water corrections. These errors are discussed in the calibration report. A combination plot is also included to facilitate correlation. A neutron moisture $\log$ of neutron counts is also shown on the combination plot.

\section{Results and Interpretations:}

${ }^{137} \mathrm{Cs}$ was the only man-made radionuclide detected. ${ }^{137} \mathrm{Cs}$ activity was detected at the three points near the ground surface. The measured ${ }^{137} \mathrm{Cs}$ activity ranged from 0.4 to $1.4 \mathrm{pCi} / \mathrm{g}$ and is interpreted as surface contamination. A marginal peak at $1408 \mathrm{keV}$ was observed at $140 \mathrm{ft}$ (file B0037026); this peak is interpreted as the ${ }^{238} \mathrm{U}\left({ }^{214} \mathrm{Bi}\right) 1407.98-\mathrm{keV}$ peak, not the ${ }^{152} \mathrm{Eu} 1408.01-\mathrm{keV}$ peak, because it is in a caliche layer with a high ${ }^{238} \mathrm{U}$ content.

The changes in gross gamma counts depend primarily upon changes in ${ }^{40} \mathrm{~K}$ activities. The increase in gross gamma counts from about $85 \mathrm{cps}$ to about $130 \mathrm{cps}$ at a log depth of $53 \mathrm{ft}$ corresponds with an increase in apparent ${ }^{40} \mathrm{~K}$ activity from about 13 to $19 \mathrm{pCi} / \mathrm{g}$. This increase in total gamma is interpreted as the Hanford $\mathrm{H} 2$. The increase in ${ }^{232} \mathrm{Th}$ activity from about 0.8 to $1.2 \mathrm{pCi} / \mathrm{g}$ and the increase in gross gamma counts from 125 to $145 \mathrm{cps}$ at $124 \mathrm{ft}$ probably represent the top of the Early Palouse Soil. On the basis of low K-40 activities, the carbonate-rich paleosols of the Pliocene-Pleistocene are interpreted as being between $139 \mathrm{ft}$ and $143 \mathrm{ft}$. The caliche layer with characteristically high uranium content (greater than $2.0 \mathrm{pCi} / \mathrm{g}$ ) is present between 140 and $144 \mathrm{ft}$. The top of the Ringold is picked at $146 \mathrm{ft}$.

Below $224 \mathrm{ft}$, the apparent increase in ${ }^{238} \mathrm{U}$ activity based on the $609-\mathrm{keV}$ spectral line of about $1 \mathrm{pCi} / \mathrm{g}$ is greater than the apparent increase in ${ }^{238} \mathrm{U}$ activity based on the $1764-\mathrm{keV}$ line of about $.25 \mathrm{pCi} / \mathrm{g}$. This apparent increase in ${ }^{238} \mathrm{U}$ at groundwater is probably the result of dissolved radon $\left({ }^{222} \mathrm{Rn}\right)$ in the water. Quantification of naturally occurring ${ }^{238} \mathrm{U}$ is based on measurement of the daughter ${ }^{214} \mathrm{Bi}$, assuming that secular equilibrium has been attained. However, ${ }^{214} \mathrm{Bi}$ is also a short-term daughter of ${ }^{222} \mathrm{Rn}$. The presence of ${ }^{222} \mathrm{Rn}$ is indicated by elevated counts in spectral peaks associated with ${ }^{214} \mathrm{~Pb}$ and ${ }^{214} \mathrm{Bi}$ and does not indicate an increase in ${ }^{238} \mathrm{U}$. The fact that a discrepancy exists between the ${ }^{214} \mathrm{Bi}$ lines at 609 and $1764 \mathrm{keV}$ suggests that radon and its daughters are present within the water. The apparent concentration based on the $609-\mathrm{keV}$ peak appears to increase more than that based on the $1764-\mathrm{keV}$ peak because the water correction factor decreases with increasing energy level. If the source of the gamma photons is within the water, then there is less attenuation than would be expected, and the effect of the water correction is an apparent increase in the calculated concentration.

The neutron moisture tool's depressed response in this hole is due to the low-activity source, and short source-to-detector spacing. The highest neutron counts occurred in the groundwater as expected. The 
elevated neutron counts per second that occur at about 125 through $140 \mathrm{ft}$ correspond with an interval of relatively high total gamma interpreted as the Early Palouse Soil.

${ }^{1}$ GWL - groundwater level

${ }^{2}$ TOC - top of casing

${ }^{3}$ N/A - not avaiłable

${ }^{4} \mathrm{n} / \mathrm{a}$ - not applicable 


\section{9-W19-45 (C3394) \\ Man-Made Radionuclide Concentrations}

${ }^{137} \mathrm{Cs}(662 \mathrm{keV})$

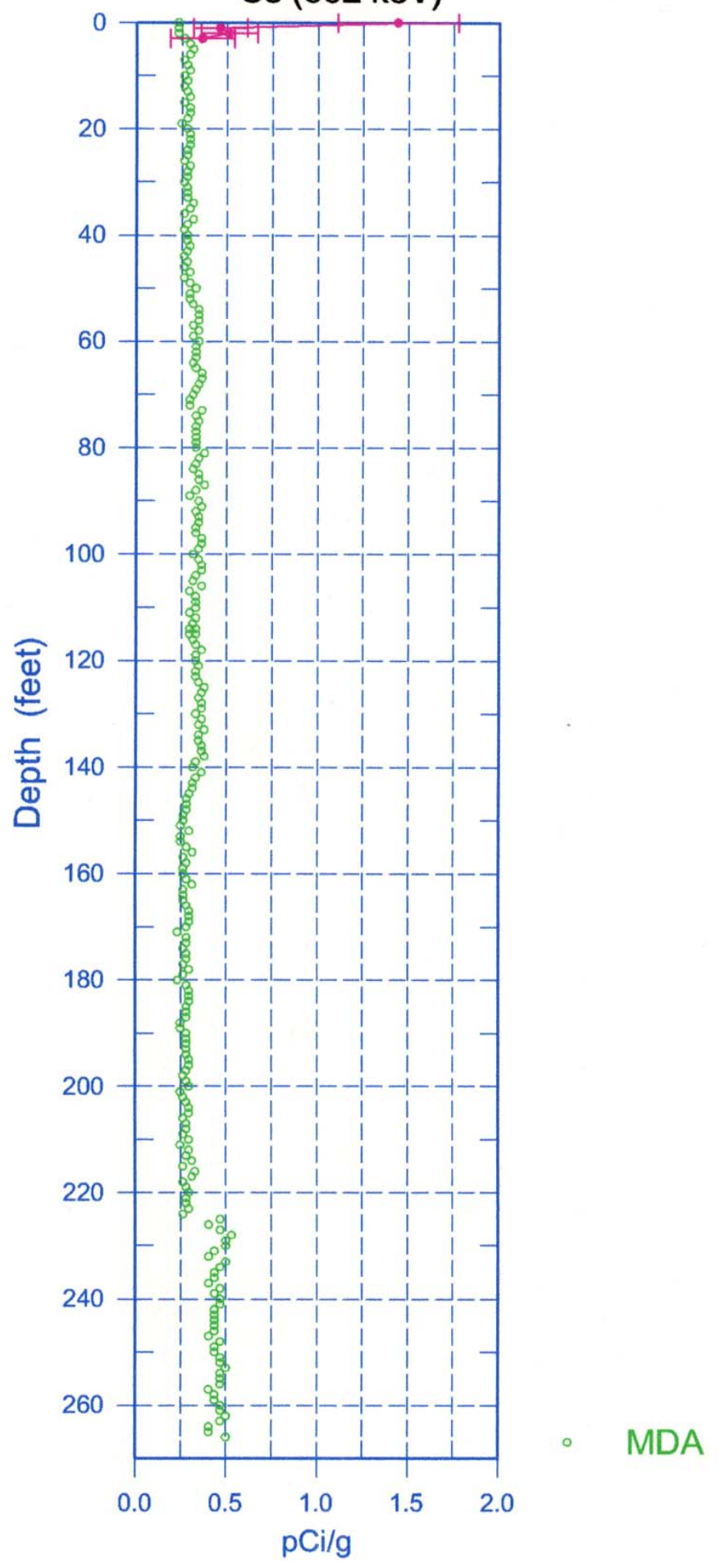

C.37 


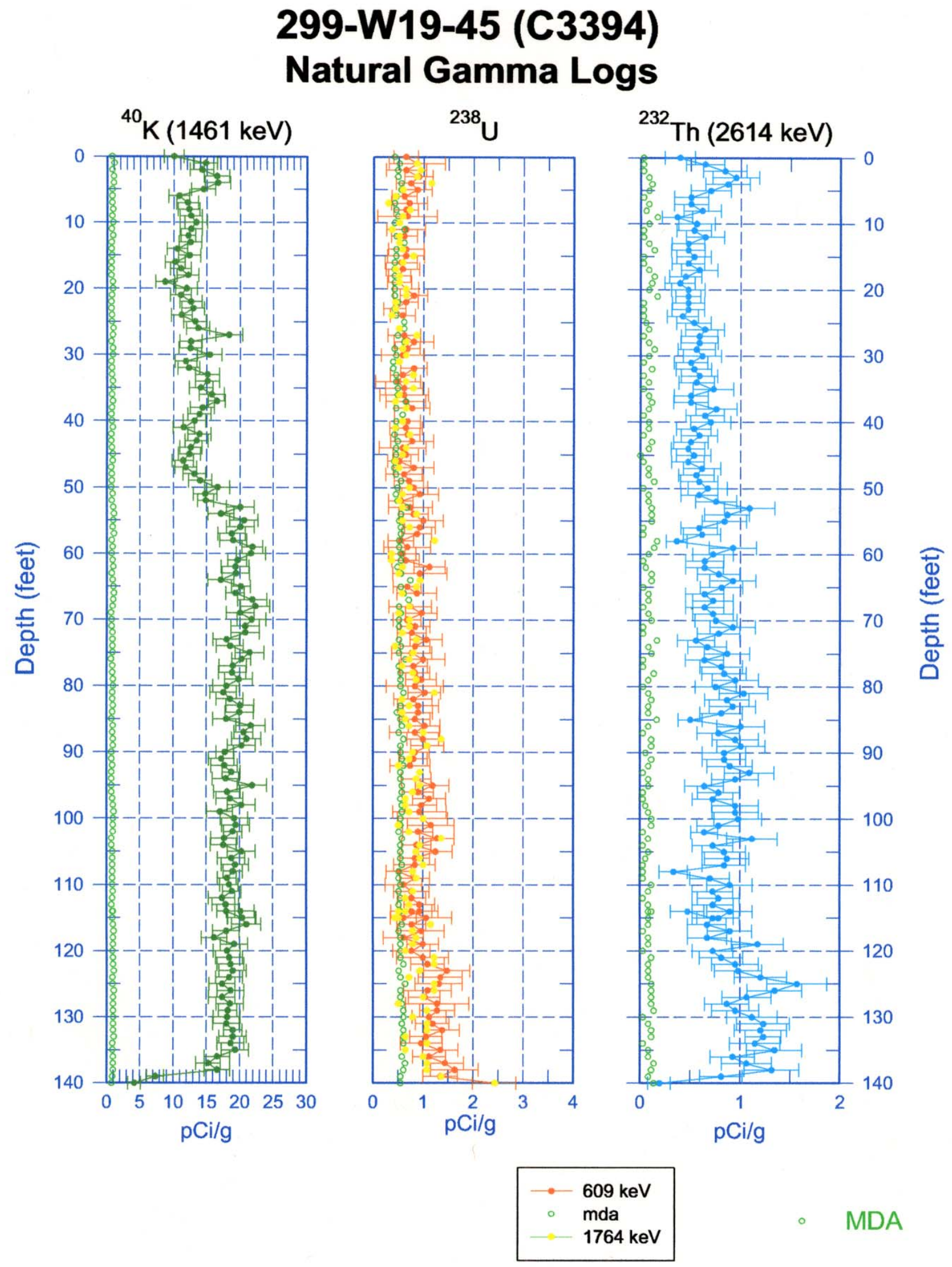

C. 38 


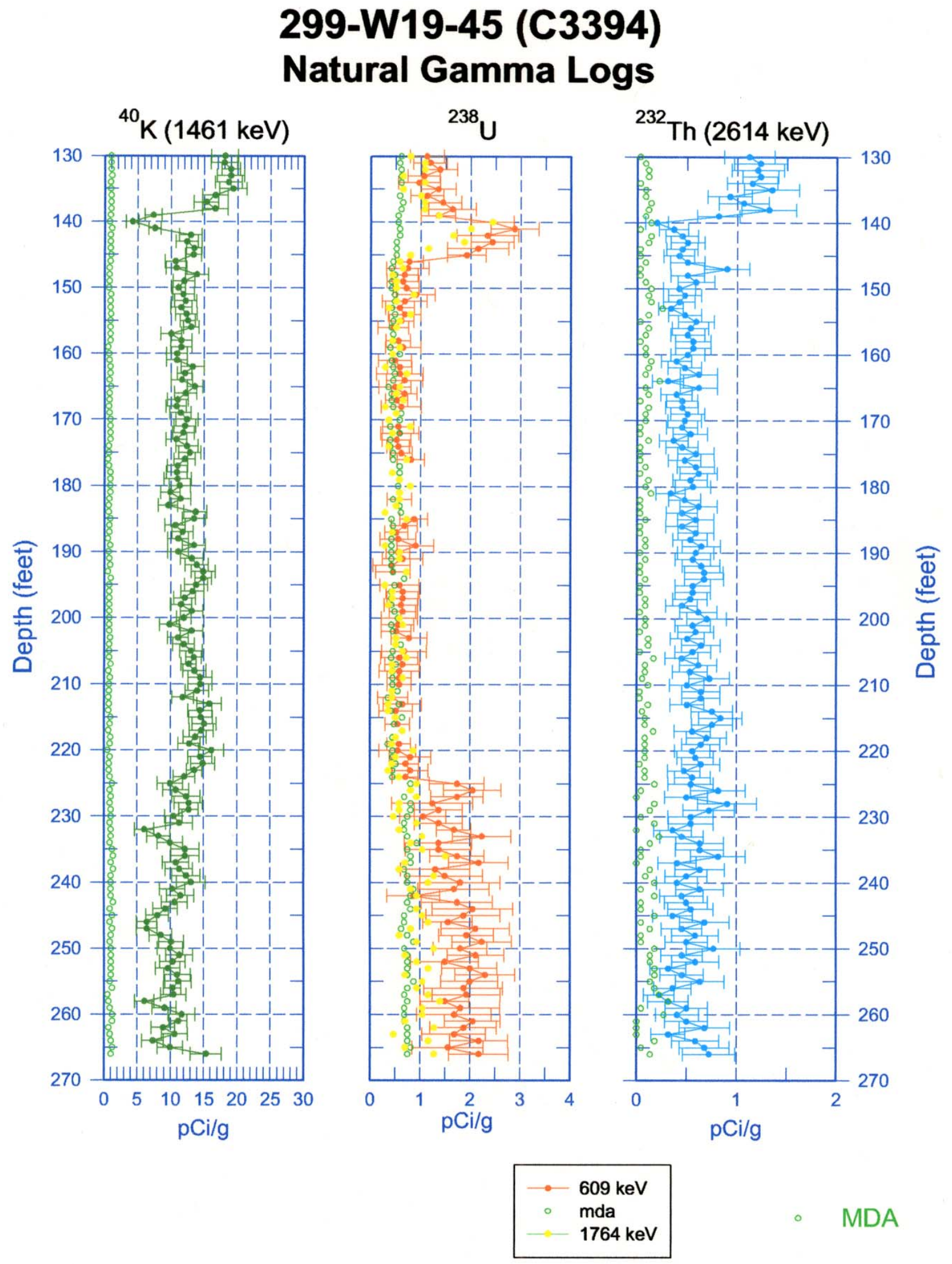

C. 39 


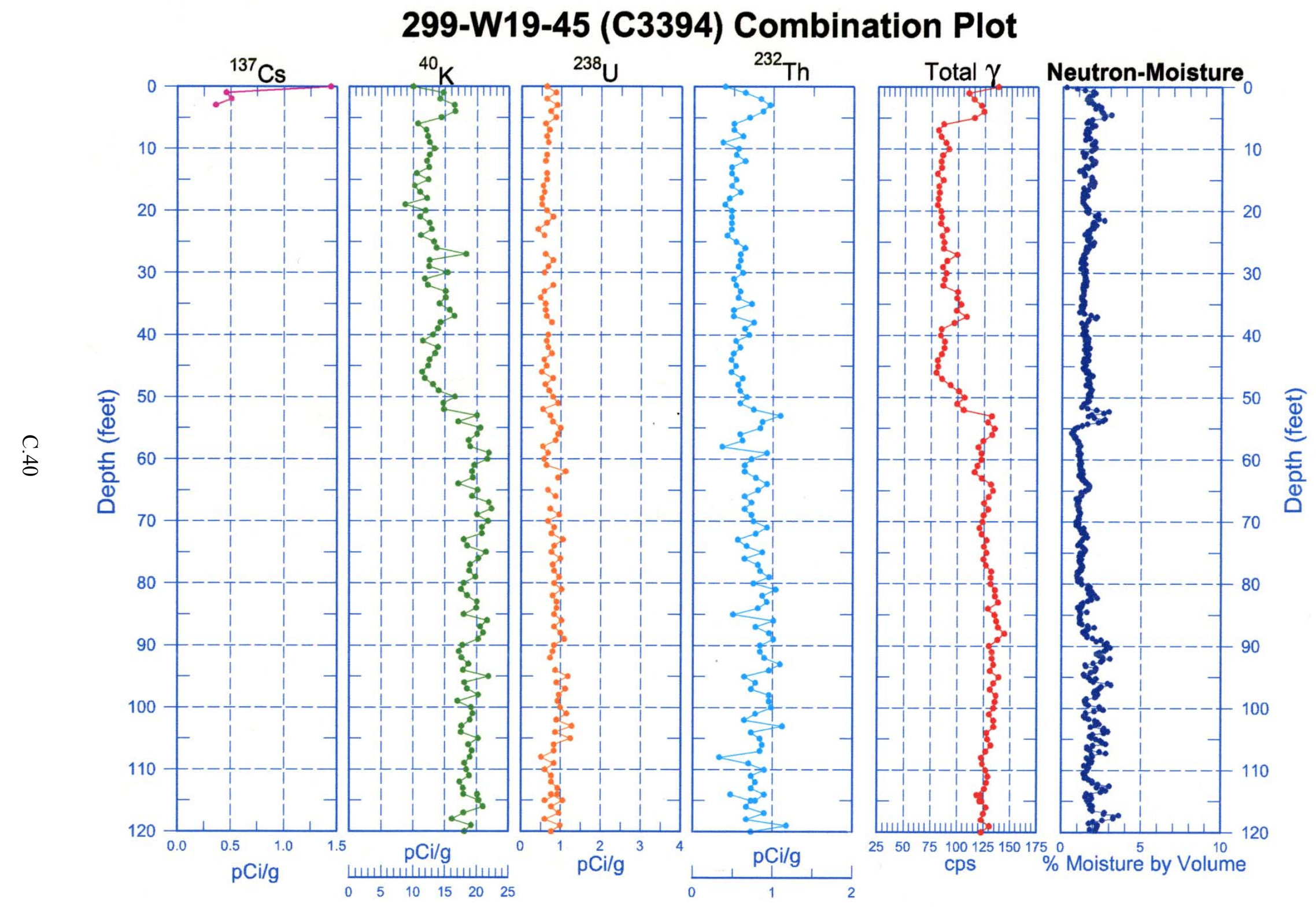




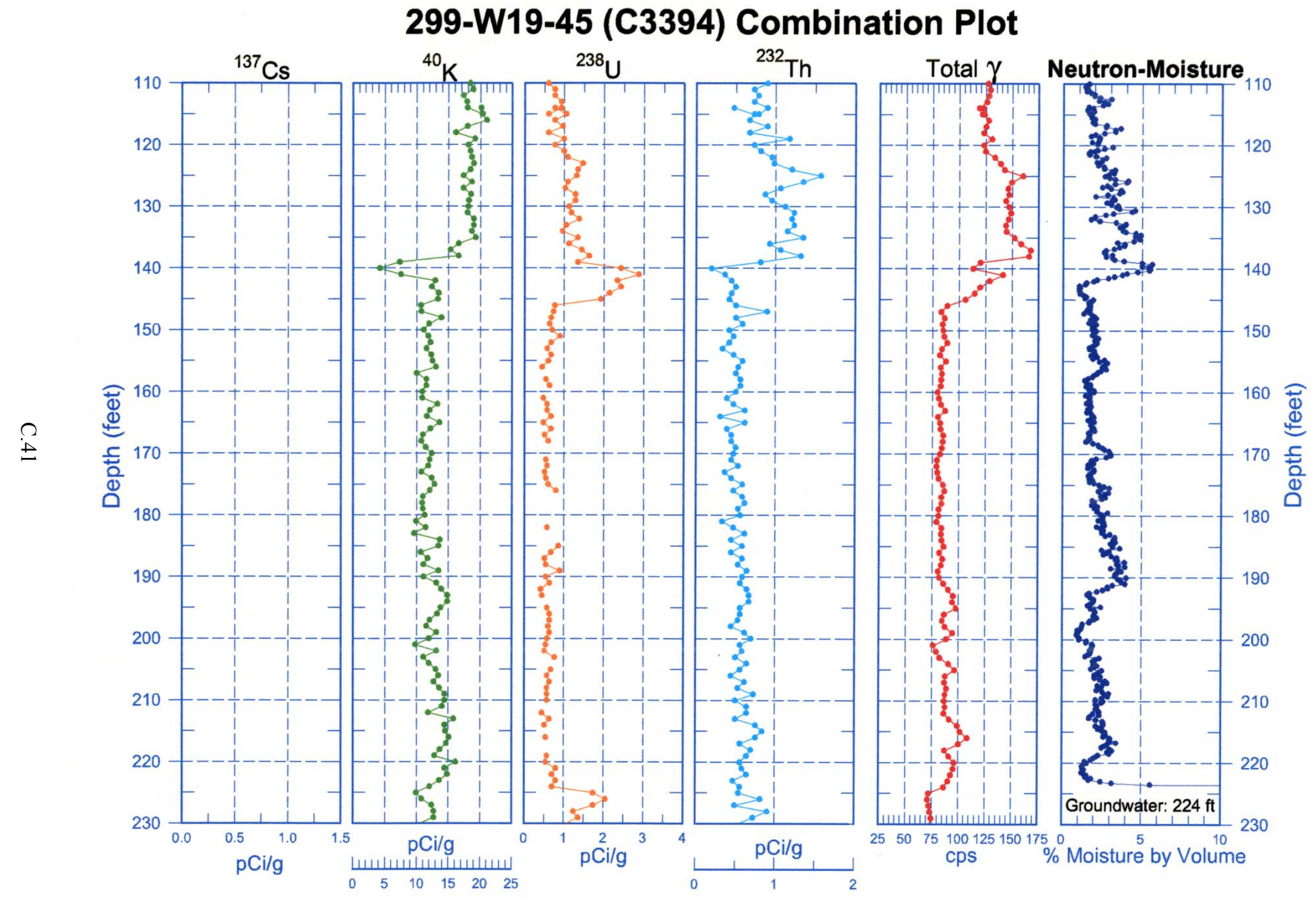




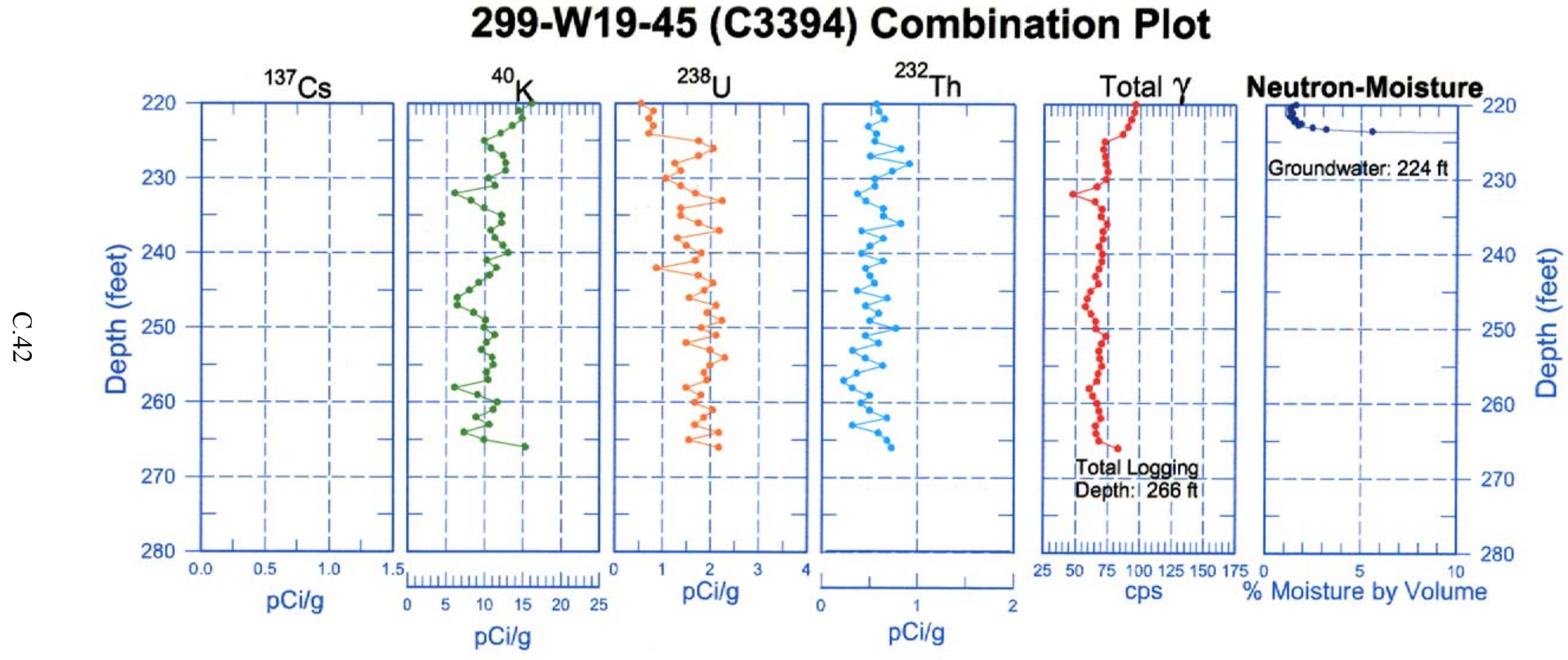




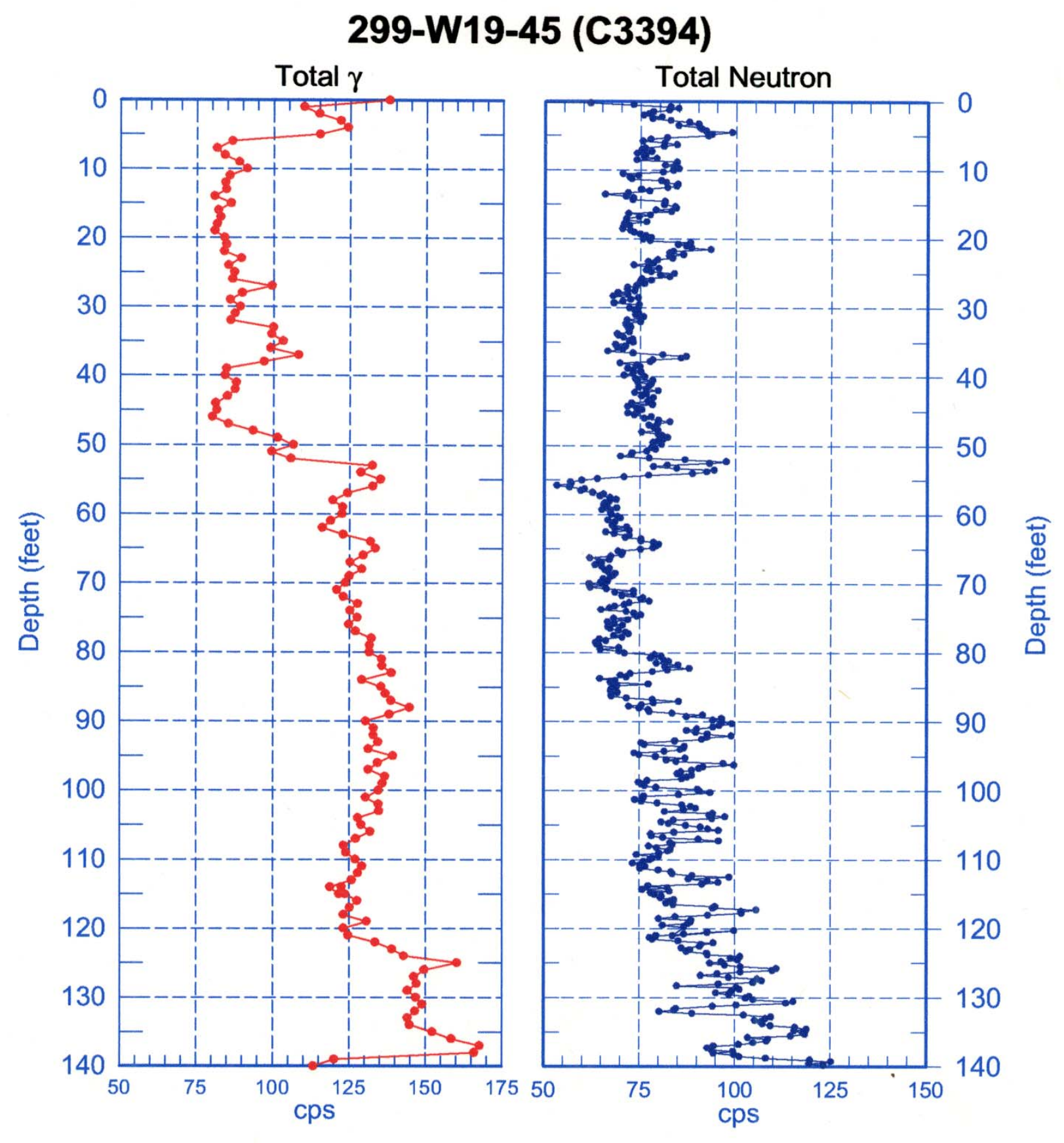




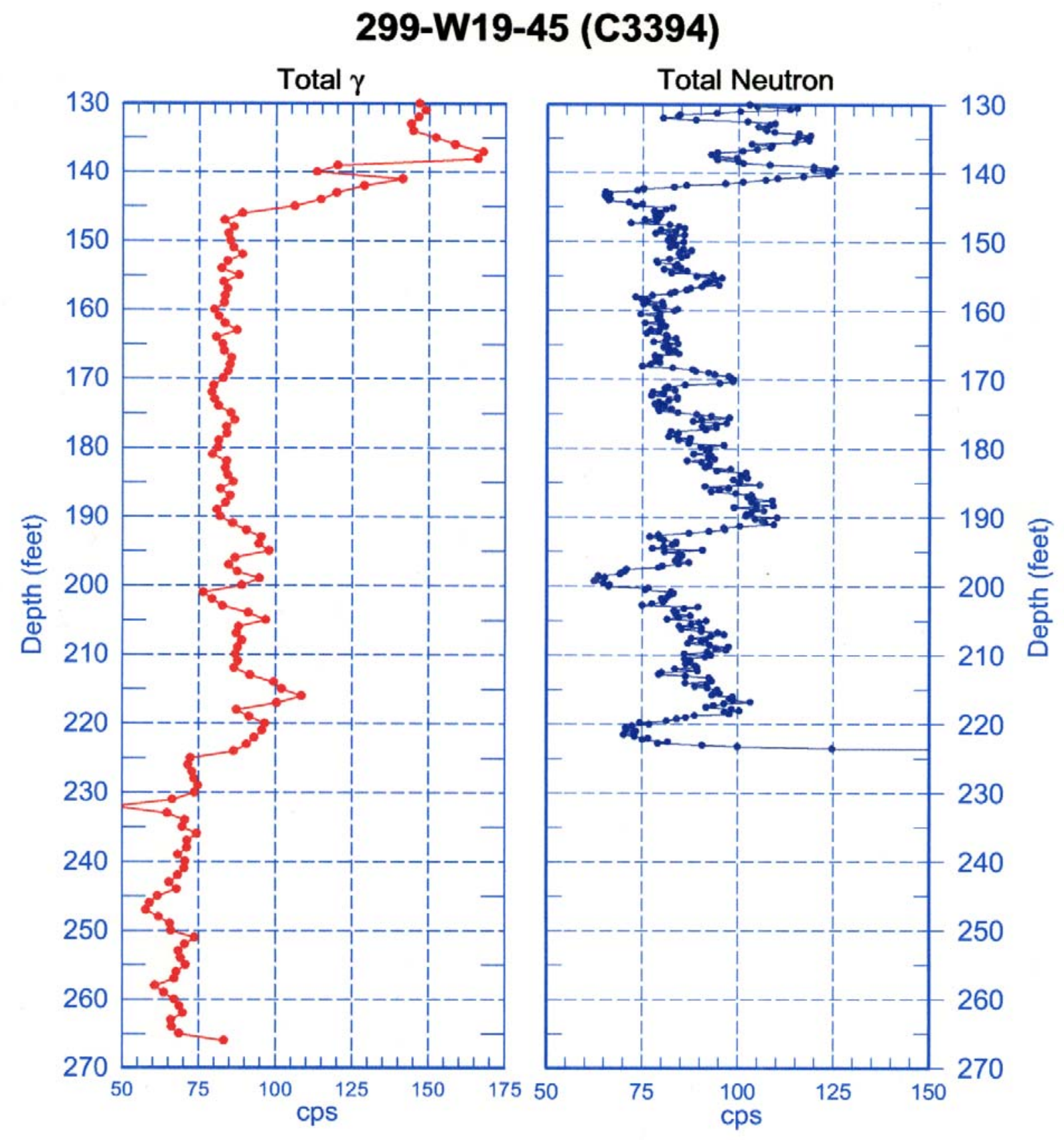




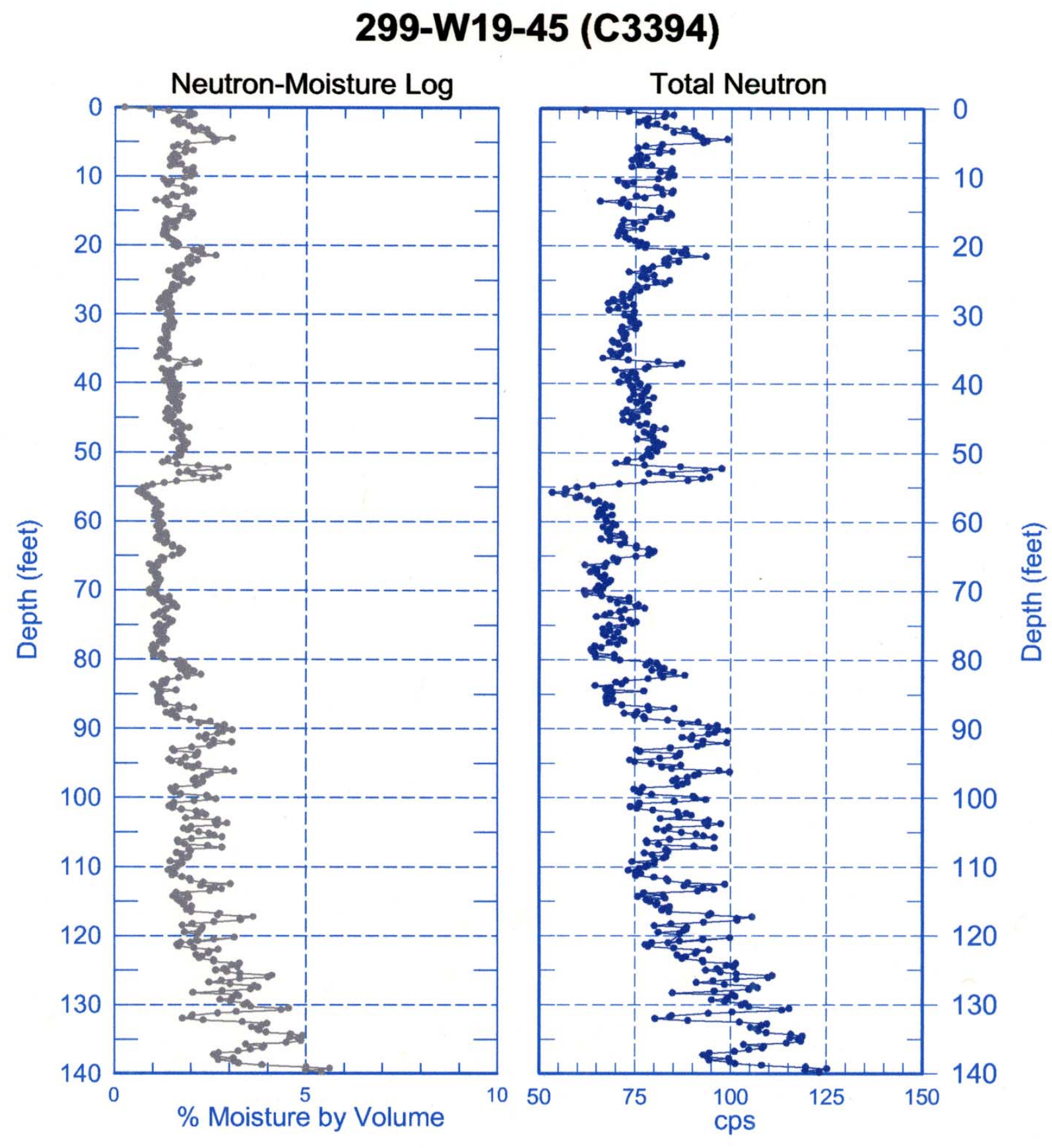




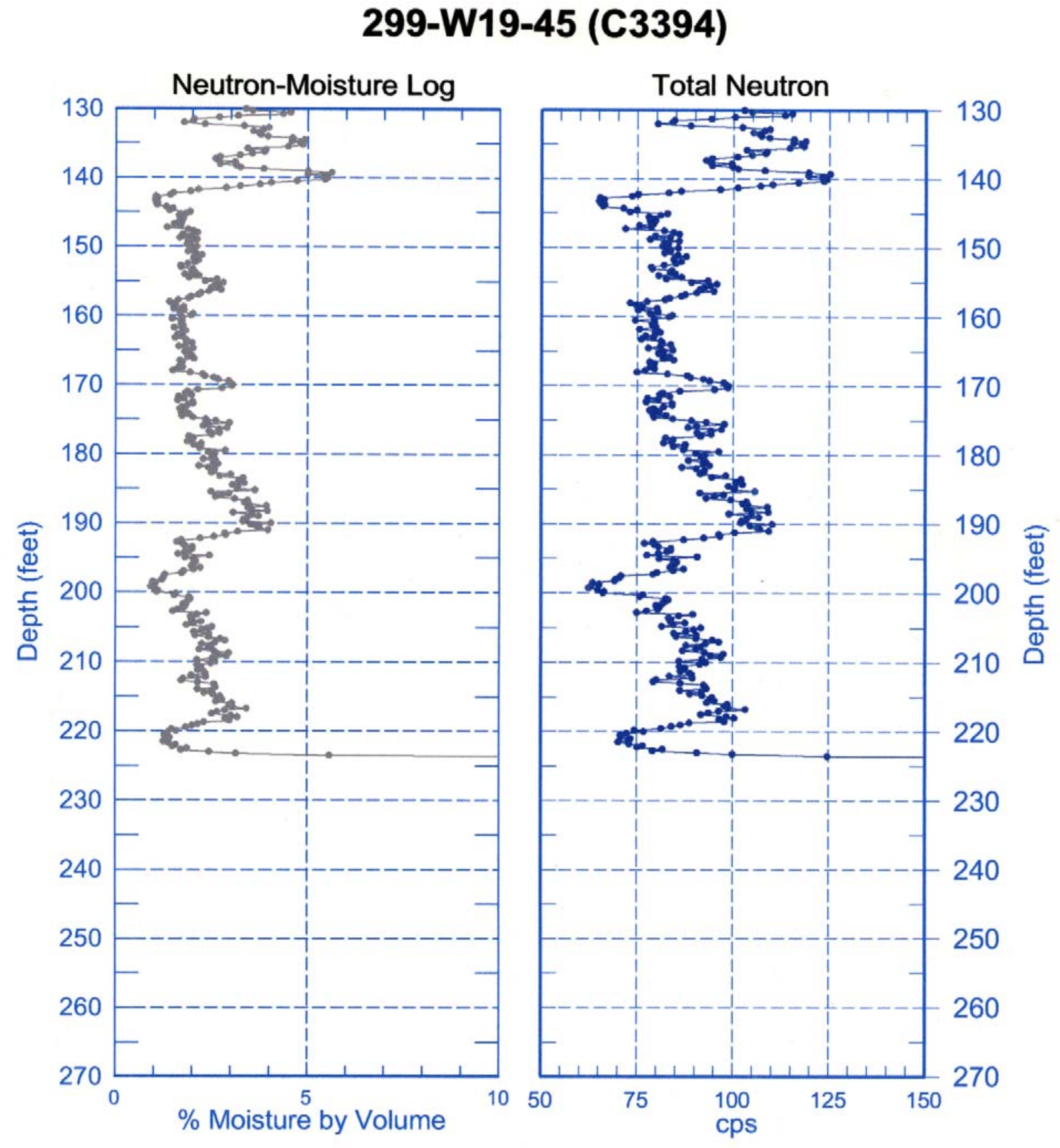

C. 46 


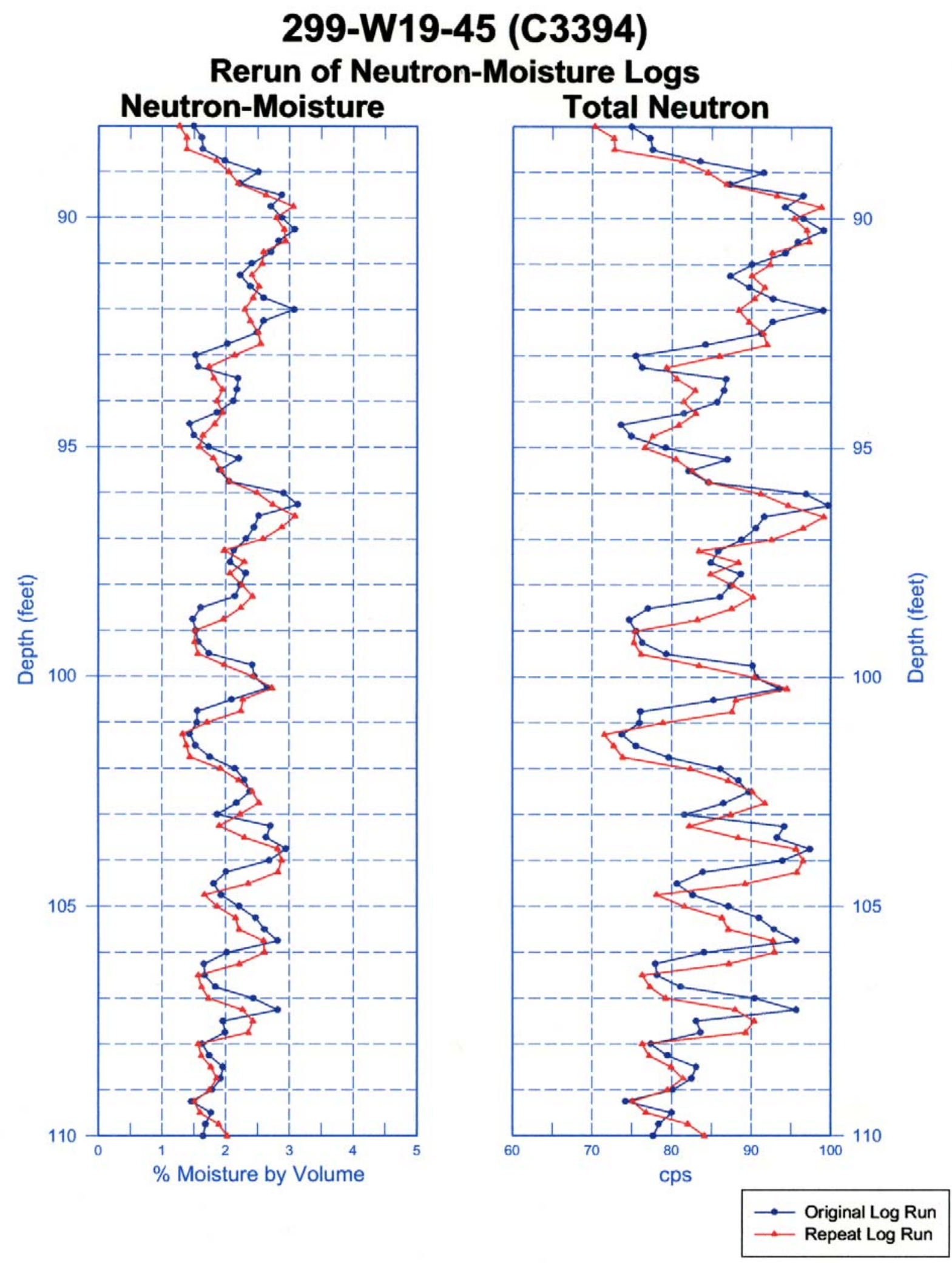

C. 47 


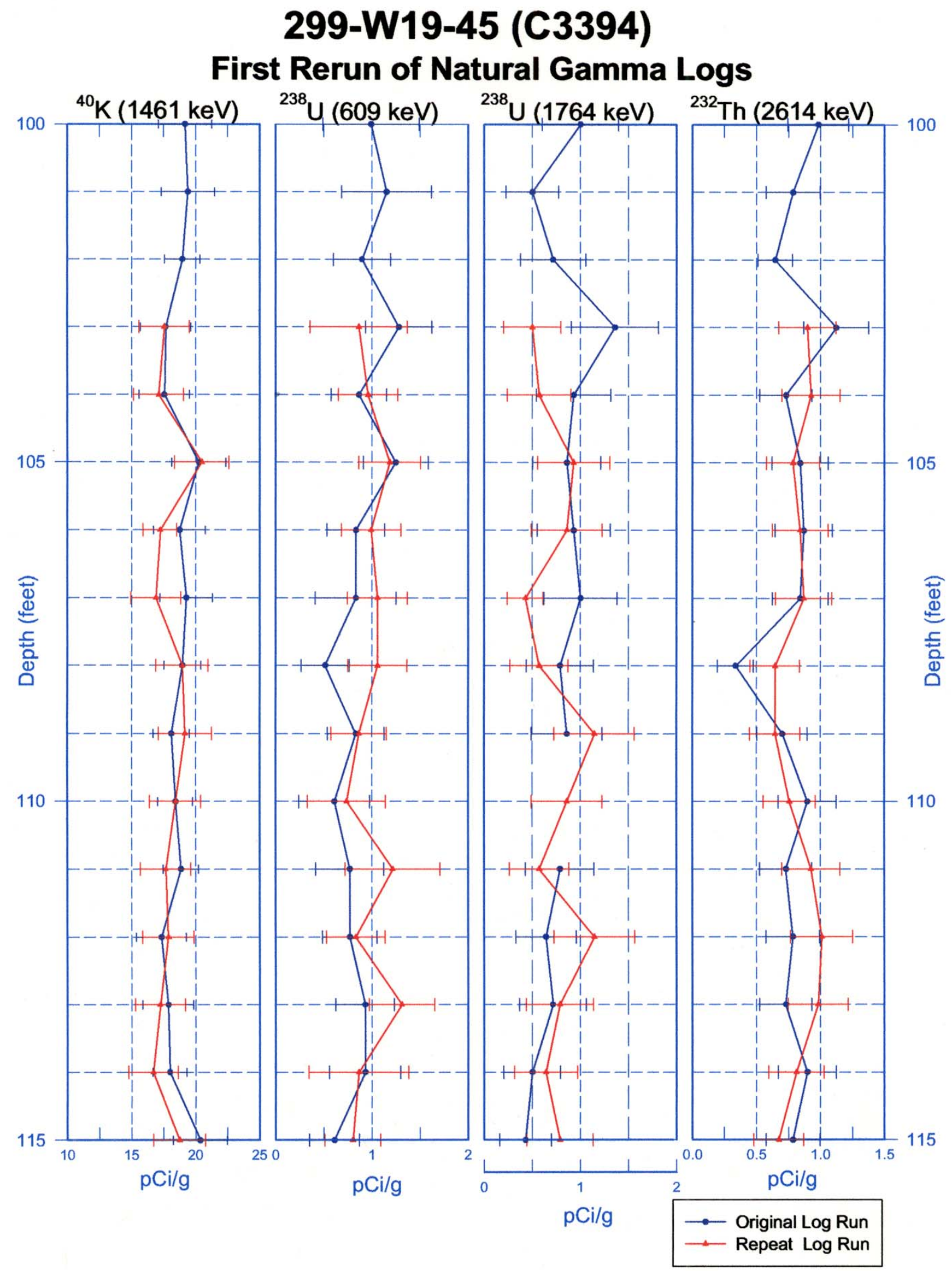

C. 48 


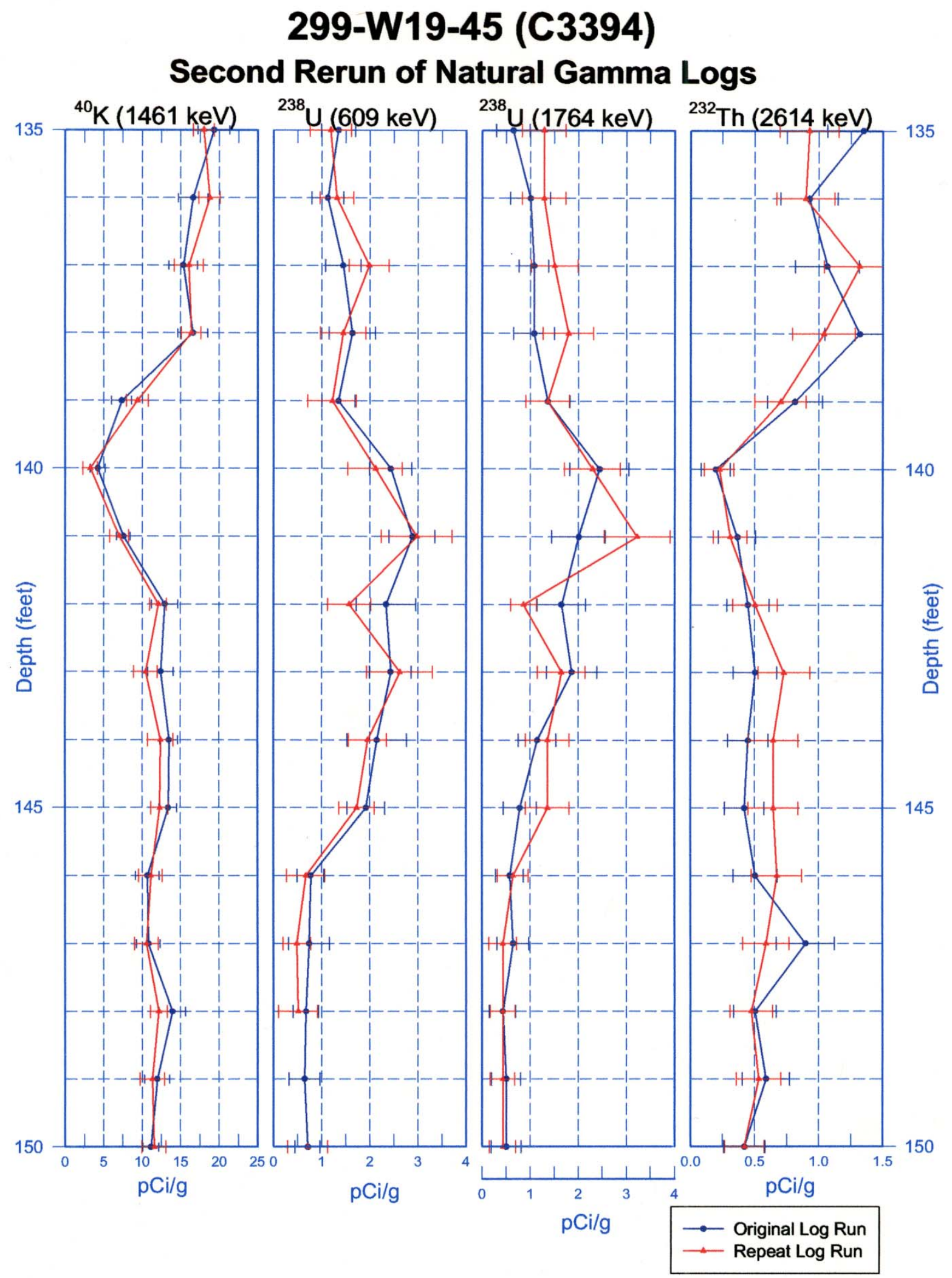

C. 49 


\section{Distribution}

No. of

\section{Copies}

OFFSITE

R. Jim

Confederated Tribes and Bands of the Yakama Nation

Environmental Restoration/Waste

Management

2808 Main Street

Union Gap, WA 98903

T. Repasky

Confederated Tribes of the Umatilla Indian

Reservation

Environmental Planning/Rights Protection

P.O. Box 638

Pendleton, OR 97801

L. Seelatsee

Wanapum Band

Grant County P.U.D.

30 "C" Street S.W.

P.O. Box 878

Ephrata, WA 98823

P. Sobotta

Nez Perce Tribe

Environmental Restoration/Waste

Management

P.O. Box 365

Lapwai, ID 83540-0365

\section{ONSITE}

\section{DOE Richland Operations Office}

M. J. Furman (2)

A5-13

R. M. Yasek

H6-60
No. of

Copies

3 CH2M HILL Hanford Group, Inc.
A. J. Knepp (2)
$\mathrm{H} 0-22$
D. A. Myers
$\mathrm{H} 0-22$

2 CH2M HILL Hanford, Inc.
J. V. Borghese
H9-03

D. C. Weekes

H9-02

\section{U.S. Environmental Protection Agency}

D. A. Faulk

B5-01

4 Washington State Department of Ecology

M. Brown

B5-18

J. A. Caggiano

B5-18

D. Goswami

B5-18

A. D. Huckaby

B5-18

14 Pacific Northwest National Laboratory
E. P. Dresel
K9-96
D. G. Horton (3)
K6-81
S. P. Luttrell
K6-96
W. J. Martin
K6-81
R. M. Smith (3)
K6-96
F. A. Spane
K6-96
D. Vela
K6-96
B. A. Williams
K6-81
Hanford Technical Library (2) 